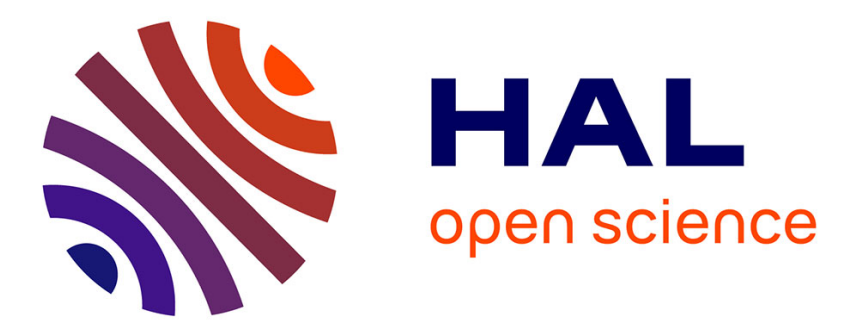

\title{
Steady sliding frictional contact problem for a 2d elastic half-space with a discontinuous friction coefficient and related stress singularities
}

Patrick Ballard

\section{- To cite this version:}

Patrick Ballard. Steady sliding frictional contact problem for a 2 d elastic half-space with a discontinuous friction coefficient and related stress singularities. Journal of the Mechanics and Physics of Solids, 2016, 97, pp.225-259. 10.1016/j.jmps.2016.03.021 · hal-02326962

\section{HAL Id: hal-02326962 \\ https://hal.science/hal-02326962}

Submitted on 22 Oct 2019

HAL is a multi-disciplinary open access archive for the deposit and dissemination of scientific research documents, whether they are published or not. The documents may come from teaching and research institutions in France or abroad, or from public or private research centers.
L'archive ouverte pluridisciplinaire HAL, est destinée au dépôt et à la diffusion de documents scientifiques de niveau recherche, publiés ou non, émanant des établissements d'enseignement et de recherche français ou étrangers, des laboratoires publics ou privés. 


\title{
Steady sliding frictional contact problem for a $2 \mathrm{~d}$ elastic half-space with a discontinuous friction coefficient and related stress singularities
}

\author{
Patrick Ballard \\ Laboratoire de Mécanique et d'Acoustique, CNRS, \\ 31, chemin Joseph Aiguier, \\ 13402 Marseille Cedex 20, France.
}

\begin{abstract}
The steady sliding frictional contact problem between a moving rigid indentor of arbitrary shape and an isotropic homogeneous elastic half-space in plane strain is extensively analysed. The case where the friction coefficient is a step function (with respect to the space variable), that is, where there are jumps in the friction coefficient, is considered. The problem is put under the form of a variational inequality which is proved to always have a solution which, in addition, is unique in some cases. The solutions exhibit different kinds of universal singularities that are explicitly given. In particular, it is shown that the nature of the universal stress singularity at a jump of the friction coefficient is different depending on the sign of the jump.
\end{abstract}

Keywords: elasticity, contact, friction, singularity, variational inequality, uniqueness

2010 MSC: 74B05, 74M10, 74M15

\section{Background: steady sliding frictional contact in linear elasticity}

The problem governing the equilibrium of a deformable body that is pressed against a frictionless obstacle in the framework of linearised elasticity is the so-called Signorini problem. Formally, the problem consists in finding a displace-

Email address: ballard@lma.cnrs-mrs.fr (Patrick Ballard) 
s ment field $\mathbf{u}: \Omega \rightarrow \mathbb{R}^{d}(d=2,3)$ such that:

$$
\mid \begin{array}{ll}
\operatorname{div} \boldsymbol{\sigma}(\mathbf{u})+\mathbf{f}^{\mathrm{p}}=\mathbf{0}, & \text { in } \Omega, \\
\mathbf{u}=\mathbf{u}^{\mathrm{p}}, & \text { on } \Gamma_{\mathrm{u}}, \\
\mathbf{t} \stackrel{\text { def }}{=} \boldsymbol{\sigma} \cdot \mathbf{n}=\mathbf{t}^{\mathrm{p}}, & \text { on } \Gamma_{\mathrm{t}}, \\
u_{\mathrm{n}}-g^{\mathrm{p}} \leq 0, \quad t_{\mathrm{n}} \leq 0, \quad\left(u_{\mathrm{n}}-g^{\mathrm{p}}\right) t_{\mathrm{n}}=0, & \\
\mathbf{t}_{\mathrm{t}}=\mathbf{0}, & \text { on } \Gamma_{\mathrm{c}},
\end{array}
$$

where $\Omega$ denotes some smooth bounded open set in $\mathbb{R}^{d}$ (the so-called stress-free reference configuration), $\Gamma_{\mathrm{u}} \cup \Gamma_{\mathrm{t}} \cup \Gamma_{\mathrm{c}}=\partial \Omega$ denotes a splitting of the boundary into three disjoint parts, and $\mathbf{n}$ is the outward unit normal. As usual, $\mathbf{u}$ is the (unknown) displacement, $\boldsymbol{\sigma}(\mathbf{u})$ is the Cauchy stress associated with this o displacement by the linear elastic constitutive law, and $\mathbf{t}=\boldsymbol{\sigma} \cdot \mathbf{n}$ denotes the surface traction. Any vector field $\mathbf{v}$ defined on part of the boundary can be split into its normal and tangential parts: $\mathbf{v}=v_{\mathrm{n}} \mathbf{n}+\mathbf{v}_{\mathrm{t}}$. The loading conditions are defined by $\mathbf{u}^{\mathrm{p}}$ (the surface displacement prescribed on $\Gamma_{\mathrm{u}}$ ), $\mathbf{t}^{\mathrm{p}}$ (the surface tractions prescribed on $\Gamma_{\mathrm{t}}$ ), $\mathbf{f}^{\mathrm{p}}$ (the prescribed body forces), and $g^{\mathrm{p}}$ (the initial

15 gap with the obstacle measured algebraically along the outward unit normal $\mathbf{n}$ to $\partial \Omega$ ).

The existence and uniqueness of a solution was first proved in 1964 by Gaetano Fichera who inspired the theory of variational inequalities which was subsequently developed in the celebrated article [1] of Jacques-Louis Lions and 20 Guido Stampacchia. Naturally, the need to integrate the handling of dry friction in the theory immediately emerged [2]. But, the so-called Signorini problem with Coulomb friction faced huge mathematical difficulties and the mathematical structure of the problem has not been completely elucidated yet. A good account of today's state of art is to be found in [3].

25 An easier contact problem involving dry friction is the steady sliding frictional contact problem which was first studied in [4]. It is the problem of the equilibrium of a (linearly) elastic body against a moving obstacle, the geometry of which remaining invariable with respect to time. In $\mathbb{R}^{2}$, such a situation is met with a straight obstacle moving along the direction of its boundary or with an obstacle so whose boundary is a circle rotating around its center (in that case, the obstacle can be either the disk or the region outside the circle). In $\mathbb{R}^{3}$, this situation encompasses the case where the boundary of the obstacle is any surface of revolution rotating around its revolution axis but there are other cases such as the case of a rotating infinite screw. Dry friction between the moving obstacle 35 and the elastic body is assumed and a displacement field in the elastic body, that is independent of time, is sought. This situation is far simpler than that of the Signorini problem with Coulomb friction because of the motion of the obstacle. The Signorini problem with Coulomb friction is doubly a free boundary problem because the contact zone (the points of the boundary that are in contact with

40 the obstacle) is unknown and also within the contact zone, the sticking zone (the points of the contact zone that adhere to the obstacle) is also unknown. In the 
steady sliding frictional contact problem, all the points in the contact zone are slipping along the obstacle since it is moving and the sliding velocity is known in advance. Considering the intersection of the outward normal to the body with the obstacle, the motion of the body results in a given slip velocity field $\mathbf{w}$ on $\Gamma_{\mathrm{c}}$ and the steady sliding frictional contact problem is formally that of finding a displacement field $\mathbf{u}: \Omega \rightarrow \mathbb{R}^{d}(d=2,3)$ such that:

$$
\mid \begin{array}{ll}
\operatorname{div} \boldsymbol{\sigma}(\mathbf{u})+\mathbf{f}^{\mathrm{p}}=\mathbf{0}, & \text { in } \Omega, \\
\mathbf{u}=\mathbf{u}^{\mathrm{p}}, & \text { on } \Gamma_{\mathrm{u}}, \\
\mathbf{t} \stackrel{\text { def }}{=} \boldsymbol{\sigma} \cdot \mathbf{n}=\mathbf{t}^{\mathrm{p}}, & \text { on } \Gamma_{\mathrm{t}}, \\
u_{\mathrm{n}}-g^{\mathrm{p}} \leq 0, \quad t_{\mathrm{n}} \leq 0, \quad\left(u_{\mathrm{n}}-g^{\mathrm{p}}\right) t_{\mathrm{n}}=0, & \\
\mathbf{t}_{\mathrm{t}}=-f t_{\mathrm{n}} \mathbf{w}_{\mathrm{t}} /\left|\mathbf{w}_{\mathrm{t}}\right|, & \text { on } \Gamma_{\mathrm{c}},
\end{array}
$$

where $f \geq 0$ is the given friction coefficient. In the case $f=0$, the Signorini problem is recovered. Therefore, the steady sliding frictional contact problem can formally be seen as a generalization of the Signorini problem in which the unknown reaction force is no longer directed along the normal to the boundary but along a given direction which is possibly slanted with respect to the normal (see figure 1).
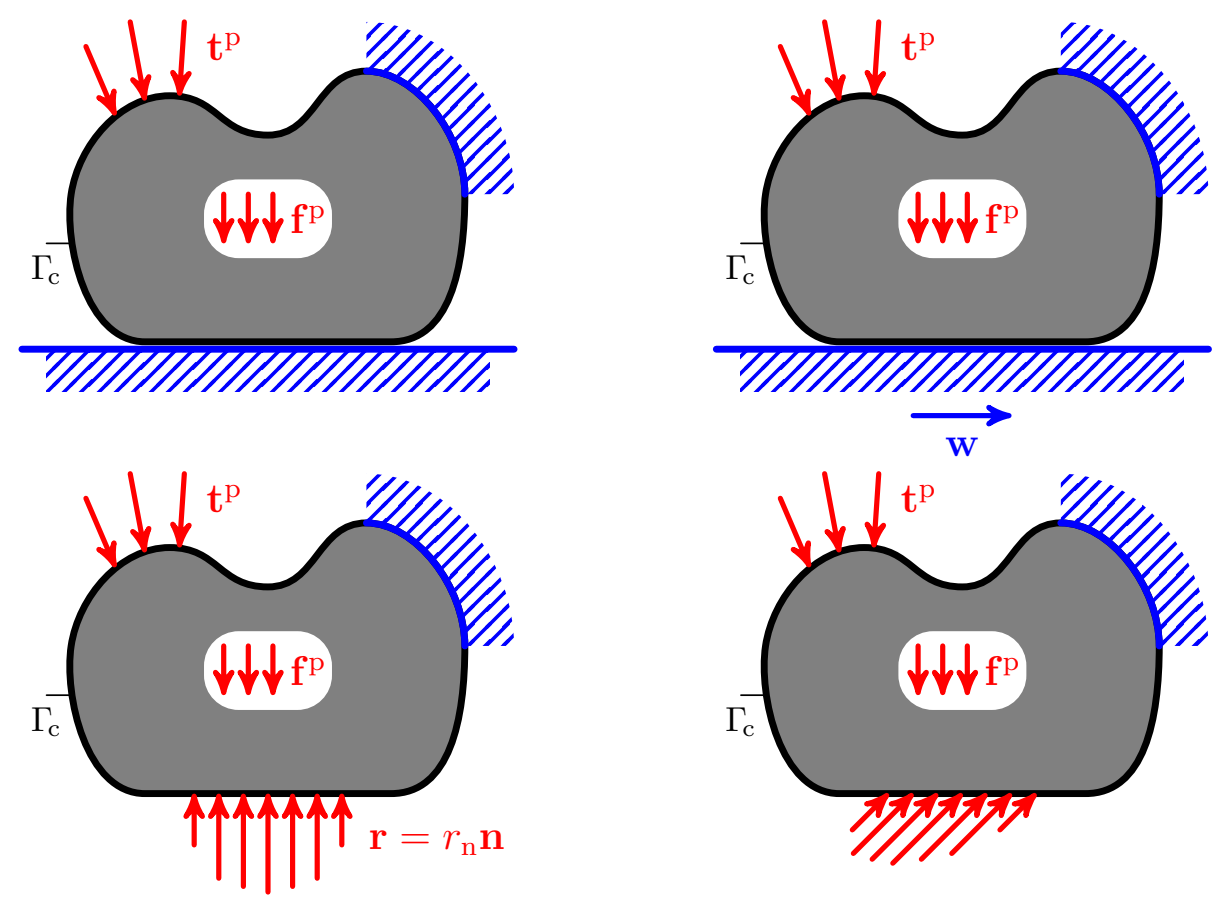

Figure 1: The Signorini problem (left) and the steady sliding frictional contact problem (right) 
This situation is very common in practice, and any machine which is supposed to run steadily provides an example. However, the situation of, say, a rubber wiper blade sliding on a glass windshield, suggests that although steady motion is generally encountered, unsteady motion, often called 'stick-slip' motion can also happen. The understanding and therefore ultimately the prediction of that 'stickslip' phenomenon is still a challenge nowadays. In [4], the structure of the set of

6o solution for the steady sliding frictional contact problem was studied for the first time. Under appropriate regularity assumptions, it was proved that the problem has a unique solution for small values of the friction coefficient. It was also proved that in the case of a bounded body, multiple solutions can be encountered for large values of the friction coefficient. Hence, the set of equilibrium solutions

${ }_{65}$ for the steady sliding frictional contact problem may exhibit a bifurcation when the friction coefficient is taken as a parameter and this bifurcation can possibly account for the occurrence of the 'stick-slip' phenomenon. Let us also mention that the static bifurcation is only one possible mechanism to account for 'stickslip' and a flutter-type mechanism can also be possible and the latter should certainly be considered to account for brake squeal for example.

In [4], the particular case where the elastic body is an isotropic elastic halfspace in plane strain was also considered. A variational inequality governing the steady sliding frictional contact problem was derived and studied. It was proved to be uniquely solvable for all values of the friction coefficient. Therefore,

75 the above-mentioned bifurcation does not occur in the case of the half-space. Actually, the situation of the half-space appears to be a favourable framework to study more thoroughly the mathematical structure of the steady sliding frictional contact problem.

In [4], the analysis of the steady sliding frictional contact problem was so restricted to the situation where the friction coefficient is a given constant. However, in the case of a composite elastic body, one should expect to have the friction coefficient which is only a step (that is, piecewise constant) function with respect to the space variable. But, the extension of the analysis in [4] to this new circumstance is not straightforward at all and raises many technical difficulties.

${ }_{85}$ It is the reason why the analysis of the steady sliding frictional contact problem with piecewise constant friction coefficient is first performed in this article in the favourable case where the elastic body is an isotropic elastic half-space in plane strain.

\section{Summary of the results that will be proved in this article}

The results obtained from the analysis performed in this article convey strong (and sometimes rather nonintuitive) mechanical information, which certainly has practical consequences. However, proving them precisely requires many technicalities and sophisticated mathematical tools. The aim of this section is to have the statement of these results made as simple as possible with a lot of 95 the mathematics disarmed, for the convenience of the reader.

We consider an isotropic homogeneous linearly elastic two-dimensional halfspace defined by $z>0$. The Poisson ratio is denoted by $\nu \in]-1,1 / 2[$ and the 
force unit is chosen so that the Young modulus $E=1$. We denote by $x$ the space variable along the boundary and by $\mathbf{t}(x)$ the surface traction distribution on the boundary and the normal and tangential components will be addressed as $t_{\mathrm{n}}(x)$ and $t_{\mathrm{t}}(x)$. Prescribing a vanishing stress field at infinity and setting:

$$
\left.\overline{\mathbf{u}}=\frac{\mathbf{u}}{2\left(1-\nu^{2}\right)}, \quad \text { and } \quad \gamma=\frac{1-2 \nu}{2(1-\nu)} \in\right] 0,3 / 4[,
$$

the surface displacement resulting from a given surface traction distribution $\mathbf{t}(x)$ is given (see for example [5] for a proof) by:

$$
\begin{aligned}
\bar{u}_{\mathrm{n}}^{\prime}(x)+\Omega & =\frac{1}{\pi} \oint_{-1}^{1} \frac{t_{\mathrm{n}}\left(x^{\prime}\right)}{x^{\prime}-x} \mathrm{~d} x^{\prime}-\gamma t_{\mathrm{t}}(x), \\
\bar{u}_{\mathrm{t}}^{\prime}(x) & =\frac{1}{\pi} \oint_{-1}^{1} \frac{t_{\mathrm{t}}\left(x^{\prime}\right)}{x^{\prime}-x} \mathrm{~d} x^{\prime}+\gamma t_{\mathrm{n}}(x),
\end{aligned}
$$

where the sign $\oint$ recalls that the integral should be understood in terms of the Cauchy principal value, and where the real constant $\Omega$ represents an arbitrary overall rotation, which will be taken as 0 in the sequel. The surface displacement is obtained up to an arbitrary additive constant, which is interpreted as being a rigid motion. This arbitrary rigid motion cannot be fixed by prescribing appropriate conditions at infinity since the displacement field is generally infinite at infinity.

Consider some rigid obstacle, the geometry of which is defined by the equation $110-z=\psi(x)(x \in]-1,1[)$, moving at a constant velocity $w>0$ along $x$, which is assumed to be parallel to the boundary of the half-space (see figure 2). Set:

$$
\bar{\psi}=\frac{\psi}{2\left(1-\nu^{2}\right)} .
$$

The steady sliding frictional contact problem was introduced and studied in [4]. In the reference frame moving with the indentor, it is formally that of finding $\mathbf{t}(x), \overline{\mathbf{u}}(x):]-1,1[\rightarrow \mathbb{R}$ such that:

- $\frac{1}{\pi} \oint_{-1}^{1} \frac{t_{\mathrm{n}}\left(x^{\prime}\right)}{x^{\prime}-x} \mathrm{~d} x^{\prime}-\gamma t_{\mathrm{t}}(x)=\bar{u}_{\mathrm{n}}^{\prime}(x)$,

- $\frac{1}{\pi} \oint_{-1}^{1} \frac{t_{\mathrm{t}}\left(x^{\prime}\right)}{x^{\prime}-x} \mathrm{~d} x^{\prime}+\gamma t_{\mathrm{n}}(x)=\bar{u}_{\mathrm{t}}^{\prime}(x)$,

- $\bar{u}_{\mathrm{n}} \leq \bar{\psi}, \quad t_{\mathrm{n}} \leq 0, \quad\left(\bar{u}_{\mathrm{n}}-\bar{\psi}\right) t_{\mathrm{n}} \equiv 0$,

- $t_{\mathrm{t}}=-f t_{\mathrm{n}}$,

- $\int_{-1}^{1} t_{\mathrm{n}}\left(x^{\prime}\right) \mathrm{d} x^{\prime}=-P$ 
where $P>0$ is the given normal component of the prescribed total force exerted on the moving obstacle, $f \geq 0$ is a given friction coefficient. Note that if $\bar{\psi}$ is changed into $\bar{\psi}+C$, then we get a solution for the new problem by just changing $\bar{u}_{\mathrm{n}}$ into $\bar{u}_{\mathrm{n}}+C$ in the solution. This means that the penetration of the indentor into the half-space is undefined and this is due to the fact that the displacement field is infinite at infinity. The problem can be parametrized by the total force $P$ only, and not by the height of the moving obstacle, because it is undetermined. This fact is intimately connected with the fact that the stress field in the half-space is not square integrable: the elastic energy of the solution is infinite and this is the reason why the problem has to be brought to the boundary by use of the fundamental solution of the Neumann problem for the half-space (the so-called Boussinesq solution). Focusing on the normal components, this

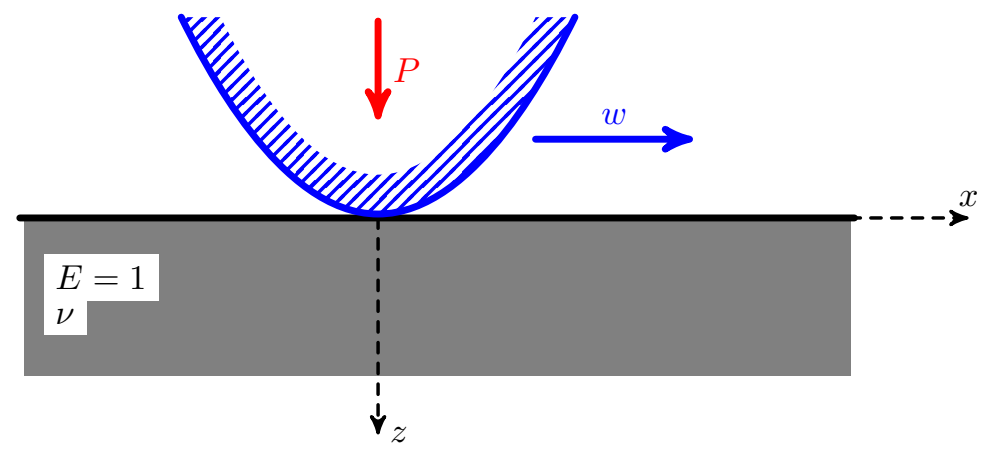

Figure 2: Geometry of the problem.

formal problem reduces to that of finding $\left.t_{\mathrm{n}}(x), \bar{u}_{\mathrm{n}}(x):\right]-1,1[\rightarrow \mathbb{R}$ such that:

$$
\begin{aligned}
& \text { - } \frac{1}{\pi} \oint_{-1}^{1} \frac{t_{\mathrm{n}}\left(x^{\prime}\right)}{x^{\prime}-x} \mathrm{~d} x^{\prime}+\gamma f(x) t_{\mathrm{n}}(x)=\bar{u}_{\mathrm{n}}^{\prime}(x), \\
& \text { - } \bar{u}_{\mathrm{n}} \leq \bar{\psi}, \quad t_{\mathrm{n}} \leq 0, \quad\left(\bar{u}_{\mathrm{n}}-\bar{\psi}\right) t_{\mathrm{n}} \equiv 0 \\
& \text { - } \quad \int_{-1}^{1} t_{\mathrm{n}}\left(x^{\prime}\right) \mathrm{d} x^{\prime}=-P .
\end{aligned}
$$

In this article, the following theorem will be proved. It shows the unconditional existence of a solution of the steady sliding frictional contact problem for any piecewise constant friction coefficient.

Theorem 1. Let $P>0$ be a positive real constant, $\bar{\psi}:]-1,1[\rightarrow \mathbb{R}$ be a piecewise Lipschitz-continuous function and $f:]-1,1[\rightarrow \mathbb{R}$ be a piecewise constant function. There exist a Radon measure $t_{\mathrm{n}} \in \mathscr{M}([-1,1])$ and a function 
$\bar{u}_{\mathrm{n}} \in H^{1 / 2}(-1,1)$ such that:

$$
\begin{aligned}
& \text { - } \left.\frac{1}{\pi} \oint_{-1}^{1} \frac{t_{\mathrm{n}}\left(x^{\prime}\right)}{x^{\prime}-x}+\gamma f t_{\mathrm{n}}=\bar{u}_{\mathrm{n}}^{\prime}, \quad \text { in }\right]-1,1[, \\
& \text { - } \bar{u}_{\mathrm{n}} \leq \bar{\psi}, \quad t_{\mathrm{n}} \leq 0, \quad\left(\bar{u}_{\mathrm{n}}-\bar{\psi}\right) t_{\mathrm{n}} \equiv 0, \\
& \text { - } \int_{-1}^{1} t_{\mathrm{n}}=-P .
\end{aligned}
$$

In addition, if $f$ is nondecreasing, $t_{\mathrm{n}}$ and $\bar{u}_{\mathrm{n}}$ are unique.

To be precise, the first equation in theorem 1 holds in the sense of distributions over ] $-1,1$ [ and the Cauchy principal value integral is defined to be the distributional derivative of the convolution product $-t_{\mathrm{n}} * \log$ (where the measure $t_{\mathrm{n}}$ is extended by zero on $\left.\mathbb{R} \backslash[-1,1]\right)$.

Proof. This a straightforward adaptation of the proofs of theorems 16 and 23 .

In the case where $\bar{\psi} \equiv 0$ (moving rigid flat punch of finite width), it is possible to derive an explicit formula for $t_{\mathrm{n}}$, for any step function $f$, by use of theorem 36 in appendix C. We shall now give this formula in two cases.

1. The friction coefficient is a constant denoted by $f$. In that case, the explicit solution of the above problem seems to have been first given by Galin [6]. It reads as:

$$
t_{\mathrm{n}}(x)=-\frac{P \cos \pi \alpha}{\pi} \frac{1}{(1+x)^{\frac{1}{2}+\alpha}(1-x)^{\frac{1}{2}-\alpha}},
$$

140

where:

$$
\alpha \stackrel{\text { def }}{=} \frac{1}{\pi} \arctan (\gamma f)=\frac{1}{\pi} \arctan \left(\frac{(1-2 \nu) f}{2(1-\nu)}\right) \quad(\in[0,1 / 2[) .
$$

This distribution of the normal component of the surface traction is represented in figure 3 . It has power singularities at both edges. The rear part of the punch is more loaded than the front part, and consistently, the rear singularity is stronger than the front one.

1452 . The friction coefficient has the constant value $f_{-}$on $]-1,0[$ and the constant value $f_{+}$on $] 0,1[$. The explicit formula in that case is derived by use of theorem 36 in appendix $\mathrm{C}$ and seems to be new. It reads as:

$$
t_{\mathrm{n}}(x)=-\frac{P}{\pi \sqrt{1+\gamma^{2} f^{2}(x)}} \frac{|x|^{\beta}}{(1+x)^{\frac{1}{2}+\alpha_{-}}(1-x)^{\frac{1}{2}-\alpha_{+}}},
$$

where:

$$
\begin{gathered}
\alpha_{-} \stackrel{\text { def }}{=} \frac{1}{\pi} \arctan \left(\gamma f_{-}\right)=\frac{1}{\pi} \arctan \left(\frac{(1-2 \nu) f_{-}}{2(1-\nu)}\right) \quad(\in[0,1 / 2[) \\
\alpha_{+} \stackrel{\text { def }}{=} \frac{1}{\pi} \arctan \left(\gamma f_{+}\right)=\frac{1}{\pi} \arctan \left(\frac{(1-2 \nu) f_{+}}{2(1-\nu)}\right) \quad(\in[0,1 / 2[) \\
\beta \stackrel{\text { def }}{=} \frac{1}{\pi} \arctan \left(\gamma f_{-}\right)-\frac{1}{\pi} \arctan \left(\gamma f_{+}\right) \quad(\in]-1 / 2,1 / 2[) .
\end{gathered}
$$




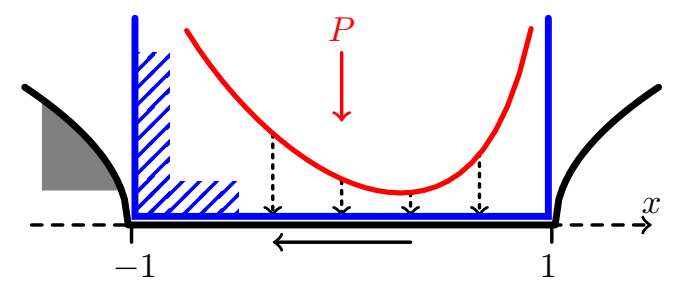

Figure 3: Normal component of the surface stress under a moving rigid flat punch with homogeneous friction coefficient.

This distribution of the normal component of the surface traction is represented in figure 4 . Its shape is very different whether the larger friction coefficient is front $\left(f_{+}\right)$or rear $\left(f_{-}\right)$. In the former case, $\beta$ is negative and the surface traction goes to infinity at the discontinuity $(x=0)$. In the latter, $\beta$ is positive and the surface traction goes to zero at the discontinuity $(x=0)$.

We have therefore discovered a fact of engineering importance. In the situation where a rigid punch with two different coatings (producing two different friction coefficients with a jump at some point) is steadily sliding along the boundary of some linearly elastic body, the way the elastic body is loaded in the vicinity of the friction coefficient discontinuity is strongly influenced by the direction along which the indentor is moving.
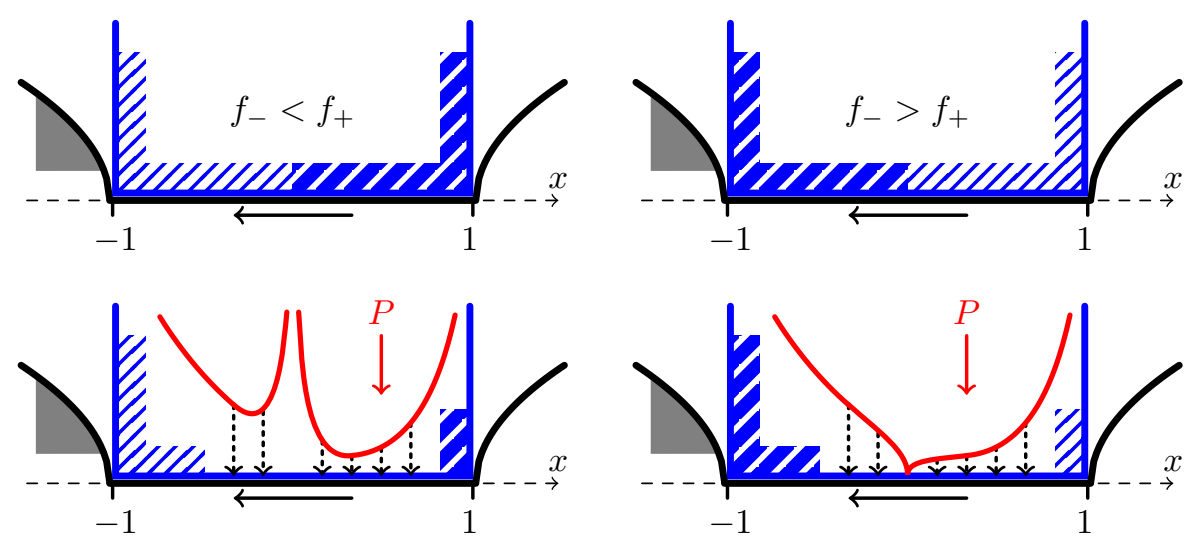

Figure 4: Normal component of the surface stress when the larger friction coefficient is front (left) or rear (right).

Another case where an explicit solution is available is the case of the moving rigid parabola with radius of curvature $\overline{\mathscr{R}} /\left(2\left(1-\nu^{2}\right)\right)$ at the minimum and with homogeneous friction coefficient $f$. A derivation of this solution which seems to have been first discovered by Galin [6] can be found in [4]. The corresponding 
function $\bar{\psi}$ reads as follows:

$$
\bar{\psi}(x)=\frac{x^{2}}{2 \overline{\mathscr{R}}} .
$$

The corresponding solution for the steady sliding frictional contact problem exhibits a connected contact zone surrounded by two detached zones. The half-diameter $a$ of the contact zone is given by:

$$
a^{2}=\frac{P \overline{\mathscr{R}}}{2 \pi\left(1 / 4-\alpha^{2}\right)},
$$

where $\alpha$ is given by formula (4). Then, provided that $P$ is small enough so that $(1+2 \alpha) a<1$, the function $t_{\mathrm{n}}$ associated with the unique solution of the corresponding problem in theorem 1 is given by:

$$
\begin{aligned}
t_{n}(x) & =\frac{-P \cos \pi \alpha}{2 \pi a^{2}\left(1 / 4-\alpha^{2}\right)}[(1+2 \alpha) a+x]^{1 / 2-\alpha}[(1-2 \alpha) a-x]^{1 / 2+\alpha}, \\
& =\frac{-1}{\overline{\mathscr{R}} \sqrt{1+\gamma^{2} f^{2}}}[(1+2 \alpha) a+x]^{1 / 2-\alpha}[(1-2 \alpha) a-x]^{1 / 2+\alpha} .
\end{aligned}
$$

The contact zone $[-(1+2 \alpha) a,(1-2 \alpha) a]$ is shifted towards the rear of the parabola and the maximum amplitude of the surface traction is reached at $x=-4 \alpha a$, which is always in the rear half of the contact zone.
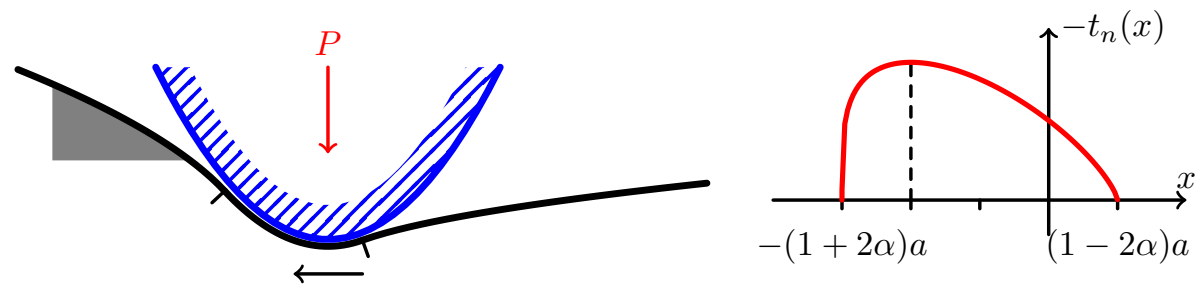

Figure 5: Rigid parabola moving at the surface of an elastic half-space.

It happens that the power singularities that have been exhibited in all the explicit solutions above are actually universal and apply to any shape of the indentor as expressed by the following theorems.

Theorem 2. Assume that $\bar{\psi}^{\prime}$ is piecewise Lipschitz-continuous. Consider a 175 solution of the problem in theorem 1 that achieves active contact in a neighborhood of the point $x=c \in]-1,1\left[\right.$. Then the measure $t_{\mathrm{n}}$ satisfies the estimate:

$$
t_{\mathrm{n}}(x)=\mid \begin{array}{ll}
\frac{1}{\gamma} \cdot \frac{\bar{\psi}^{\prime}(c+)-\bar{\psi}^{\prime}(c-)}{f(c+)-f(c-)}-\frac{\theta|c-x|^{\beta}}{\sqrt{1+\gamma^{2} f^{2}(x)}}, & \text { if } f(c+) \neq f(c-), \\
\frac{\bar{\psi}^{\prime}(c+)-\bar{\psi}^{\prime}(c-)}{1+\left((\gamma f(c))^{2}\right.}\left(\frac{\log |x-c|}{\pi}+\gamma f(c) H(x-c)\right)+\theta, & \text { otherwise, }
\end{array}
$$

for some measure $\theta$ whose restriction to a neighborhood of $c$ is a continuous function, and where $\beta$ is given by formula (7) and $H$ is the Heaviside function. 
Proof. This is a straightforward adaptation of the proofs of theorems 9, 17 and 24.

The stress singularity arising in the case of a jump in the friction coefficient seems to have never been investigated before. Stress singularities arising in other but similar situations of contact problems in linear elasticity were also investigated in [7], [8] and [5].

We shall express in words the conclusions of theorem 2, revealing how nonintuitive they are.

- In the case where the friction coefficient has no jump at $c(f(c+)=f(c-))$, but where the indentor has a wedge at $c\left(\bar{\psi}^{\prime}(c+)>\bar{\psi}^{\prime}(c-)\right)$, then the surface traction has a logarithmic singularity at $c$. Note that the coefficient of that singularity (the so-called stress intensity factor) is fully determined by the geometry of the wedge, the Poisson ratio and the friction coefficient. This fact is in sharp contrast with the usual singularities met in linear elasticity in polyhedra (see [9]) where the stress intensity factor is indeterminate and depends actually on the global problem (and not only on the local data). Needless to say, this result applies to the Signorini problem which corresponds to the particular case where $f \equiv 0$. Note that the nonpositivity condition on $t_{\mathrm{n}}(x)$ entails $\bar{\psi}^{\prime}(c+) \geq \bar{\psi}^{\prime}(c-)$. Therefore, in case of an obstacle with reentrant corner $\left(\bar{\psi}^{\prime}(c+)<\bar{\psi}^{\prime}(c-)\right)$, the vertex is never an interior point of the contact zone.

- In the case where the friction coefficient has a jump at $c(f(c+) \neq f(c-))$, but where the indentor has no wedge at $c\left(\bar{\psi}^{\prime}(c+)=\bar{\psi}^{\prime}(c-)\right)$, then the surface traction has a power singularity at $c$. The surface traction goes to infinity in the case $f(c+)>f(c-)$, but it always goes to zero in the case $f(c+)<f(c-)$.

- In the case where the friction coefficient has a jump at $c(f(c+) \neq f(c-))$, and the indentor has a wedge at $c\left(\bar{\psi}^{\prime}(c+) \neq \bar{\psi}^{\prime}(c-)\right)$, then the wedge has no effect on the power singularity in the case $f(c+)>f(c-)$, but in the case $f(c+)<f(c-)$, the surface traction has now a negative limit at $c$, instead of zero.

Theorem 3. Let $c \in[-1,1]$. We assume a constant friction coefficient $f_{-} \geq 0$ on $] c-\epsilon, c\left[\right.$ and a constant friction coefficient $f_{+} \geq 0$ on $] c, c+\epsilon[(\epsilon>0)$, if appropriate.

We consider a solution $\bar{u}_{\mathrm{n}}, t_{\mathrm{n}}$ of the problem in theorem 1 such that contact with the indentor is achieved in a right-neighbourhood of $c$ whereas detachment 215 occurs in a left-neighbourhood of c. More precisely, if one of the two following assumptions is satisfied:

- $c=-1, \bar{u}_{\mathrm{n}}=\bar{\psi}$ on $]-1,-1+\epsilon\left[(\epsilon>0)\right.$ and $\bar{\psi}^{\prime}$ is Lipschitz-continuous on ]$-1,-1+\epsilon[$,

- $c \in]-1,1\left[, \bar{\psi}(c-)>\bar{\psi}(c+), \bar{u}_{\mathrm{n}}<\bar{\psi}\right.$ on $] c-\epsilon, c\left[(\epsilon>0), \bar{u}_{\mathrm{n}}=\bar{\psi}\right.$ on ]$c, c+\epsilon\left[\right.$ and $\bar{\psi}^{\prime}$ is Lipschitz-continuous on $] c-\epsilon, c[$ and $] c, c+\epsilon[$, 
then, the solution $\bar{u}_{\mathrm{n}}, t_{\mathrm{n}}$ in theorem 1 satisfies the estimate:

$$
t_{\mathrm{n}}=-\frac{\theta_{-}}{|x-c|^{1 / 2+\alpha_{-}}},
$$

where $\alpha_{-}$is given by formula (5) and $\theta_{-}$is a measure whose restriction to a right-neighbourhood of $x=c$ is a Hölder-continuous function. In addition, in the case where $\theta_{-}(c)>0$, then the following estimate holds true:

$$
\bar{u}_{\mathrm{n}}^{\prime}(x) \sim-\frac{\theta_{-}(c) \sqrt{1+\gamma^{2} f_{-}^{2}}}{|x-c|^{1 / 2+\alpha_{-}}}, \quad \text { as } x \rightarrow c-.
$$

If the following assumption is satisfied:

- $c \in]-1,1\left[, \bar{\psi}(c-)=\bar{\psi}(c+), \bar{u}_{\mathrm{n}}<\bar{\psi}\right.$ on $] c-\epsilon, c\left[(\epsilon>0), \bar{u}_{\mathrm{n}}=\bar{\psi}\right.$ on ]$c, c+\epsilon\left[\right.$ and $\bar{\psi}^{\prime}$ is Lipschitz-continuous on $] c-\epsilon, c[$ and $] c, c+\epsilon[$,

then, the solution $\bar{u}_{\mathrm{n}}, t_{\mathrm{n}}$ in theorem 1 satisfies the estimate:

$$
t_{\mathrm{n}}=-|c-x|^{1 / 2-\alpha_{-}} \theta_{-}
$$

where $\theta_{-}$is a measure whose restriction to a right-neighbourhood of $x=c$ is a Hölder-continuous function.

Similarly, we consider a solution $\bar{u}_{\mathrm{n}}, t_{\mathrm{n}}$ of the problem in theorem 1 such that contact with the indentor is achieved in a left-neighbourhood of $c$ whereas detachment occurs in a right-neighbourhood of c. More precisely, if one of the two following assumptions is satisfied:

- $c=1, \bar{u}_{\mathrm{n}}=\bar{\psi}$ on $] 1-\epsilon, 1\left[(\epsilon>0)\right.$ and $\bar{\psi}^{\prime}$ is Lipschitz-continuous on ] $1-\epsilon, 1[$,

- $c \in]-1,1\left[, \bar{\psi}(c-)<\bar{\psi}(c+), \bar{u}_{\mathrm{n}}=\bar{\psi}\right.$ on $] c-\epsilon, c\left[(\epsilon>0), \bar{u}_{\mathrm{n}}<\bar{\psi}\right.$ on ]$c, c+\epsilon\left[\right.$ and $\bar{\psi}^{\prime}$ is Lipschitz-continuous on $] c-\epsilon, c[$ and $] c, c+\epsilon[$,

then, the solution $\bar{u}_{\mathrm{n}}, t_{\mathrm{n}}$ in theorem 1 satisfies the estimate:

$$
t_{\mathrm{n}}=-\frac{\theta_{+}}{|x-c|^{1 / 2-\alpha_{+}}},
$$

where $\alpha_{+}$is given by formula (6) and $\theta_{+}$is a measure whose restriction to a left-neighbourhood of $x=c$ is a Hölder-continuous function. In addition, in the case where $\theta_{+}(c)>0$, then the following estimate holds true:

$$
\bar{u}_{\mathrm{n}}^{\prime}(x) \sim \frac{\theta_{+}(c) \sqrt{1+\gamma^{2} f_{+}^{2}}}{|x-c|^{1 / 2-\alpha_{+}}}, \quad \text { as } x \rightarrow c-.
$$

If the following assumption is satisfied:

- $c \in]-1,1\left[, \bar{\psi}(c-)=\bar{\psi}(c+), \bar{u}_{\mathrm{n}}=\bar{\psi}\right.$ on $] c-\epsilon, c\left[(\epsilon>0), \bar{u}_{\mathrm{n}}<\bar{\psi}\right.$ on ]$c, c+\epsilon\left[\right.$ and $\bar{\psi}^{\prime}$ is Lipschitz-continuous on $] c-\epsilon, c[$ and $] c, c+\epsilon[$, 
then, the solution $\bar{u}_{\mathrm{n}}, t_{\mathrm{n}}$ in theorem 1 satisfies the estimate:

$$
t_{\mathrm{n}}=-|c-x|^{1 / 2+\alpha_{+}} \theta_{+}
$$

where $\theta_{+}$is a measure whose restriction to a left-neighbourhood of $x=c$ is a Hölder-continuous function.

Proof. This a straightforward adaptation of the proof of theorem 10.

In words, theorem 3 says that the singularity of the surface traction at a discontinuity of the indentor (a jump of $\bar{\psi}$ ) is the same power singularity, going to infinity, as the one obtained at the edge of the moving rigid flat punch. This power singularity is different whether this discontinuity is a front or a rear boundary of the contact zone. In the case of a boundary of the contact zone at a

255 smooth point of the indentor (a continuity point of $\bar{\psi}$ ), then the surface traction has a power singularity having zero as a limit, as the one obtained at the edge of the contact zone for the moving rigid parabola. This power singularity is different whether this boundary is a front or a rear boundary of the contact zone.

Remark 1. The fact that the front and the rear edges of the moving indentor 260 experiment different stress singularities was already noted in [7]. It relies strongly on the fact that $\gamma \neq 0$ and the same is true of the existence of singularities at a jump of the friction coefficient. This highlights the fact that the usual and convenient approximation which consists in neglecting the influence of friction on the normal component of the surface traction is not valid in the case of a nonsmooth indentor of a nonconstant friction coefficient, except for the limiting case of incompressibility which corresponds to the situation $\gamma \rightarrow 0$. More generally, the analysis extends readily to the case where the moving indentor is elastic (instead of rigid) by replacing $\gamma$ by Dundurs' bimaterial constant. Naturally, in the exceptional cases where Dundurs' bimaterial constant vanishes, there is no influence of friction on the normal component of the surface traction, and the analysis is driven back to the frictionless situation.

Remark 2. The analysis performed for the geometry of the half-space in this paper evidences the functional spaces that must be used to extend the analysis performed for a smooth bounded body in the case of a constant friction coefficient in [4] to the case of a piecewise constant friction coefficient. This extension still has to be written down, but in the light of [4], we must expect that the existence result will be obtained only under the condition that the maximum value of the friction coefficient is small enough. In that case, and provided that the bounded body is made of an homogenous isotropic linear elastic material, the solution 280 will display the same singularities as those described in the present paper for the half-space, as is usual in linear elasticity.

\section{Analysis of homogeneous friction}

In this section, it is assumed that the friction coefficient $f$ is a given real constant. The dual (that is, taking the surface traction as main unknown) weak 
where $C$ is an arbitrary constant. Introducing the 1-dimensional vector space:

$$
\mathscr{C} \stackrel{\text { def }}{=}\left\{C \chi_{]-1,1[} \mid C \in \mathbb{R}\right\},
$$

where $\chi_{S}$ denotes the characteristic function of the set $S$, the normal surface displacement is therefore seen to naturally belong to the quotient space $H_{0} \stackrel{\text { def }}{=}$ $H^{1 / 2}(-1,1) / \mathscr{C}$. The dual space $H_{0}^{\prime}$ is:

$$
H_{0}^{\prime}=\left\{\hat{t} \in H^{-1 / 2}(-1,1) \mid\left\langle\hat{t}, \chi_{]-1,1}\right\rangle=0\right\}
$$

and is a closed subspace of codimension 1 in $H^{-1 / 2}(-1,1)$. Furthermore, taking 310

$t_{\mathrm{n}}$ equal to:

$$
t_{0}(x) \stackrel{\text { def }}{=} \frac{\cos \pi \alpha}{\pi} \frac{1}{(1+x)^{\frac{1}{2}+\alpha}(1-x)^{\frac{1}{2}-\alpha}}
$$


with:

$$
\alpha \stackrel{\text { def }}{=} \frac{1}{\pi} \arctan (\gamma f) \quad(\in]-1 / 2,1 / 2[),
$$

in the first equation of problem (3), gives $\bar{u}_{\mathrm{n}}^{\prime} \equiv 0$ (see [5] or appendix C). Since $\int_{-1}^{1} t_{0}=1$, any $\hat{t} \in H^{-1 / 2}(-1,1)$ can be decomposed in the form:

$$
t=\tilde{t}+t_{0}\left\langle t, \chi_{]-1,1[}\right\rangle_{H^{-1 / 2}, H^{1 / 2}},
$$

where $\tilde{t} \in H_{0}^{\prime}$.

Now, given $\bar{u}_{\mathrm{n}} \in H_{0}=H^{1 / 2}(-1,1) / \mathscr{C}$, consider the problem of finding $t_{\mathrm{n}} \in H^{-1 / 2}(-1,1)$ such that:

$$
\begin{aligned}
& \left.-\frac{1}{\pi} t_{\mathrm{n}} * \log |\cdot|+\frac{\gamma f}{2} t_{\mathrm{n}} * \operatorname{sgn}(\cdot)=\bar{u}_{\mathrm{n}}, \quad \text { on }\right]-1,1[, \\
& \left\langle t_{\mathrm{n}}, \chi_{]-1,1[}\right\rangle_{H^{-1 / 2}, H^{1 / 2}}=-P .
\end{aligned}
$$

To obtain a weak formulation of that problem, we introduce the bilinear form defined on $H^{-1 / 2}(-1,1) \times H^{-1 / 2}(-1,1)$ by:

$$
b\left(\hat{t}_{1}, \hat{t}_{2}\right) \stackrel{\text { def }}{=}-\frac{1}{\pi}\left\langle\hat{t}_{1} * \log |x|, \hat{t}_{2}\right\rangle_{H^{1 / 2}, H^{-1 / 2}}+\frac{\gamma f}{2}\left\langle\hat{t}_{1} * \operatorname{sgn}(x), \hat{t}_{2}\right\rangle_{H^{1 / 2}, H^{-1 / 2}} .
$$

The restriction of $b(\cdot, \cdot)$ to $H_{0}^{\prime} \times H_{0}^{\prime}$ is continuous and coercive, for all $f \in \mathbb{R}$.

Hence, making the shift of unknown:

$$
t_{\mathrm{n}}=\tilde{t}_{\mathrm{n}}-P t_{0},
$$

the above problem (9) reduces to that of finding $\tilde{t}_{\mathrm{n}} \in H_{0}^{\prime}$ such that:

$$
\forall \hat{t} \in H_{0}^{\prime}, \quad b\left(\tilde{t}_{\mathrm{n}}, \hat{t}\right)=\left\langle\bar{u}_{\mathrm{n}}, \hat{t}\right\rangle_{H_{0}, H_{0}^{\prime}} .
$$

By the Lions-Stampacchia theorem [1] (actually, only the Lax-Milgram theorem, which is a particular case of the Lions-Stampacchia theorem, is needed), this problem has a unique solution denoted by:

$$
\tilde{t}_{\mathrm{n}}=\mathscr{L}\left(\bar{u}_{\mathrm{n}}\right),
$$

325 where $\mathscr{L}$ is a continuous linear mapping from $H_{0}$ onto $H_{0}^{\prime}$, and the unique solution $t_{\mathrm{n}}$ of problem (9) is given by:

$$
t_{\mathrm{n}}=\mathscr{L}\left(\bar{u}_{\mathrm{n}}\right)-P t_{0} .
$$

Taking $u \in W^{1, p}(-1,1)$ for some $p>1$, the problem of finding $t \in$ $\cup_{p>1} L^{p}(-1,1)$ such that:

- $\frac{1}{\pi} \oint_{-1}^{1} \frac{t\left(x^{\prime}\right)}{x^{\prime}-x} \mathrm{~d} x^{\prime}+\gamma f t(x)=u^{\prime}(x), \quad$ for a.a. $\left.x \in\right]-1,1[$,

- $\int_{-1}^{1} t\left(x^{\prime}\right) \mathrm{d} x^{\prime}=-P$. 
has a unique solution given by:

$$
\begin{array}{r}
t(x)=\frac{\gamma f u^{\prime}(x)}{1+(\gamma f)^{2}}-\frac{1}{1+(\gamma f)^{2}} \frac{1}{\pi} \oint_{-1}^{1} \frac{\left(1+x^{\prime}\right)^{\frac{1}{2}+\alpha}\left(1-x^{\prime}\right)^{\frac{1}{2}-\alpha}}{(1+x)^{\frac{1}{2}+\alpha}(1-x)^{\frac{1}{2}-\alpha} \cdot \frac{u^{\prime}\left(x^{\prime}\right)}{x^{\prime}-x} \mathrm{~d} x^{\prime}} \\
-\frac{P \cos \pi \alpha}{\pi} \frac{1}{(1+x)^{\frac{1}{2}+\alpha}(1-x)^{\frac{1}{2}-\alpha}},
\end{array}
$$

where:

$$
\alpha \stackrel{\text { def }}{=} \frac{1}{\pi} \arctan (\gamma f) \quad(\in]-1 / 2,1 / 2[),
$$

(see [5] or appendix $\mathrm{C}$ in the case $p=+\infty$ ). Therefore, the restriction of $\mathscr{L}$ to $W^{1, p}(-1,1) / \mathscr{C}(p>1)$ is given by:

$$
\mathscr{L}(u)=\frac{\gamma f u^{\prime}(x)}{1+(\gamma f)^{2}}-\frac{1}{1+(\gamma f)^{2}} \frac{1}{\pi} \oint_{-1}^{1} \frac{\left(1+x^{\prime}\right)^{\frac{1}{2}+\alpha}\left(1-x^{\prime}\right)^{\frac{1}{2}-\alpha}}{(1+x)^{\frac{1}{2}+\alpha}(1-x)^{\frac{1}{2}-\alpha}} \cdot \frac{u^{\prime}\left(x^{\prime}\right)}{x^{\prime}-x} \mathrm{~d} x^{\prime} .
$$
of $v$

$$
t \geq 0 \text { in } H^{s} / \mathscr{L} \quad \Longleftrightarrow \quad \exists l \in \mathscr{L}, \quad t+l \geq 0 \text { in } H^{s} .
$$

Finally, a distribution $t \in \mathscr{D}^{\prime}(]-1,1[)$ is said to be nonpositive (notation $t \leq 0)$, if $-t$ is nonnegative. We will also use the notations $t_{1} \leq t_{2}$ or $t_{2} \geq t_{1}$ to mean $t_{2}-t_{1} \geq 0$. 
Whenever not confusing, we will frequently use the same notation for the equivalence class $\bar{\psi} \in H_{0}=H^{1 / 2}(-1,1) / \mathscr{C}$ and one of its arbitrary element $\bar{\psi} \in H^{1 / 2}(-1,1)$. Recalling the splitting $t_{\mathrm{n}}=\tilde{t}_{\mathrm{n}}-P t_{0}$, with $\tilde{t}_{\mathrm{n}} \in H_{0}^{\prime}$, this abuse helps in making the contact conditions explicit:

$$
\begin{aligned}
\exists C \in \mathbb{R}, \quad \bar{u}_{\mathrm{n}}-\bar{\psi}+C \leq 0, \quad \tilde{t}_{\mathrm{n}}-P t_{0} \leq 0, \\
0=\left(\tilde{t}_{\mathrm{n}}-P t_{0}\right)\left(\bar{u}_{\mathrm{n}}-\bar{\psi}+C\right) \in \mathscr{D}^{\prime}(]-1,1[),
\end{aligned}
$$

which entails that $\left\langle\tilde{t}_{\mathrm{n}}-P t_{0}, \bar{u}_{\mathrm{n}}-\bar{\psi}+C\right\rangle_{H^{-1 / 2}, H^{1 / 2}}=0$.

Given $\bar{\psi} \in H_{0}$, we set:

$$
\begin{aligned}
& K_{0} \stackrel{\text { def }}{=}\left\{\hat{u} \in H_{0} \mid \hat{u}-\bar{\psi} \leq 0\right\}, \\
& K_{0}^{\prime} \stackrel{\text { def }}{=}\left\{\hat{t} \in H_{0}^{\prime} \mid \hat{t}-P t_{0} \leq 0\right\},
\end{aligned}
$$

and:

$$
\begin{aligned}
\varphi(\hat{u}) \stackrel{\text { def }}{=} \mid \begin{array}{ll}
\inf _{C \mid \hat{u}-\bar{\psi}+C \leq 0}\left\langle-P t_{0}, \hat{u}-\bar{\psi}+C\right\rangle_{H^{-1 / 2}, H^{1 / 2}}, & \text { if } \hat{u} \in K_{0}, \\
+\infty, & \text { otherwise, }
\end{array} \\
=\mid \begin{array}{ll}
\left\langle-P t_{0}, \bar{u}_{\mathrm{n}}-\bar{\psi}-\underset{3-1,1[}{\operatorname{ess} \sup (\hat{u}-\bar{\psi})\rangle_{H^{-1 / 2}, H^{1 / 2}},}\right. & \text { if } \hat{u} \in K_{0}, \\
+\infty, & \text { otherwise, }
\end{array}
\end{aligned}
$$

where the fact $P \geq 0$ was used.

Lemma 4. For $P \geq 0$, the functional $\varphi$ is proper, lower semicontinuous on $H_{0}$ and convex.

Proof. Since $\varphi(\hat{u})=\left\langle\operatorname{ess} \sup (\hat{u}-\bar{\psi})-(\hat{u}-\bar{\psi}), P t_{0}\right\rangle$, it is obvious that $\varphi$ is convex. If a sequence $\left(u_{n}\right)_{n \in \mathbb{N}}$ converges strongly in $H^{1 / 2}(-1,1)$ (or even in $\left.L^{2}(-1,1)\right)$ towards $u$ and, if:

$$
\forall n \in \mathbb{N}, \quad \text { for a.a. } x \in]-1,1\left[, \quad u_{n}(x) \leq M,\right.
$$

for some $M \in \mathbb{R}$, then:

$$
\text { for a.a. } x \in]-1,1[, \quad u(x) \leq M .
$$

Therefore:

$$
\underset{]-1,1[}{\operatorname{ess} \sup } u \leq \liminf _{n \rightarrow+\infty} \operatorname{ess} \sup u_{n},
$$

and the functional $\hat{u} \mapsto \operatorname{ess} \sup _{-1,1} \hat{u}$ is lower semicontinuous on $H^{1 / 2}(-1,1)$. 
Proposition 5. Given $P>0, \bar{u}_{\mathrm{n}} \in H_{0}$ and $\tilde{t}_{\mathrm{n}} \in H_{0}^{\prime}$, the contact conditions (11) can be equivalently rewritten as (i) or (ii) with:

$$
\begin{aligned}
& \text { (i) } \tilde{t}_{\mathrm{n}} \in K_{0}^{\prime} \quad \text { and } \quad \forall \hat{t} \in K_{0}^{\prime}, \quad\left\langle\hat{t}-\tilde{t}_{\mathrm{n}}, \bar{u}_{\mathrm{n}}-\bar{\psi}\right\rangle_{H_{0}^{\prime}, H_{0}} \geq 0, \\
& \text { (ii) } \bar{u}_{\mathrm{n}} \in K_{0} \quad \text { and } \quad \forall \hat{u} \in K_{0}, \quad\left\langle\tilde{t}_{\mathrm{n}}, \hat{u}-\bar{u}_{\mathrm{n}}\right\rangle_{H_{0}^{\prime}, H_{0}}+\varphi(\hat{u})-\varphi\left(\bar{u}_{\mathrm{n}}\right) \geq 0 .
\end{aligned}
$$

\section{Proof.}

The fact that the contact conditions (11) imply (i) is obvious. Reciprocally, assume that $(i)$ is true. Since $\tilde{t}_{\mathrm{n}} \in K_{0}^{\prime}, \tilde{t}_{\mathrm{n}}-P t_{0}$ is an element of $H^{-1 / 2}(-1,1)$ which is also a nonpositive measure. Pick $\bar{u}_{\mathrm{n}}, \bar{\psi} \in H^{1 / 2}(-1,1)$ in the respective equivalence classes. Since $t_{0} \in H^{-1 / 2}(-1,1)$, there exists a unique $C \in \mathbb{R}$ such that:

$$
P C=\left\langle\tilde{t}_{\mathrm{n}}-P t_{0}, \bar{u}_{\mathrm{n}}-\bar{\psi}\right\rangle_{H^{-1 / 2}, H^{1 / 2}},
$$

that is:

$$
\left\langle\tilde{t}_{\mathrm{n}}-P t_{0}, \bar{u}_{\mathrm{n}}-\bar{\psi}+C\right\rangle_{H^{-1 / 2}, H^{1 / 2}}=0 .
$$

Therefore:

$$
\forall \hat{t} \in K_{0}^{\prime}, \quad\left\langle\hat{t}-P t_{0}, \bar{u}_{\mathrm{n}}-\bar{\psi}+C\right\rangle_{H^{-1 / 2}, H^{1 / 2}} \geq 0 .
$$

Set:

$$
S \stackrel{\text { def }}{=} \operatorname{Supp}\left\langle\bar{u}_{\mathrm{n}}(x)-\bar{\psi}(x)+C\right\rangle^{+},
$$

where $\langle x\rangle^{+}=\max \{x, 0\}$ stands for the positive part. If the Lebesgue measure of $S$ was not equal to zero, then, choosing:

$$
\hat{t}(x)=\mid \begin{array}{ll}
P t_{0}(x), & \text { if } x \in]-1,1[\backslash S, \\
-\frac{1}{\text { meas } S} \int_{]-1,1[\backslash S} P t_{0}, & \text { if } x \in S,
\end{array}
$$

in formula (15) would lead to contradiction. Therefore meas $S=0$, that is $\bar{u}_{\mathrm{n}}-\bar{\psi}+C \leq 0$ almost everywhere in ]-1, 1[. Finally, $\bar{u}_{\mathrm{n}}-\bar{\psi}+C$ is integrable with respect to the measure $\tilde{t}_{\mathrm{n}}-P t_{0}$, thanks to formula (14), and $\left(\tilde{t}_{\mathrm{n}}-P t_{0}\right)\left(\bar{u}_{\mathrm{n}}-\bar{\psi}+C\right)$ is nothing but the null measure.

385 Let us now prove the equivalence between the contact condition (11) and statement (ii). First note that $\tilde{t}=P t_{0}$ being impossible (since $P>0$ ), the constant $C$ appearing in (11) is unique and must equal $-\operatorname{ess} \sup \left(\bar{u}_{\mathrm{n}}-\bar{\psi}\right)$. It is therefore obvious that the contact condition (11) implies statement (ii). Reciprocally, let us assume that statement (ii) holds true. Applying it with the two choices $\hat{u}=\bar{\psi}$ and $\hat{u}=\bar{\psi}+2\left(\bar{u}_{\mathrm{n}}-\bar{\psi}\right)$ gives:

$$
\left\langle\tilde{t}_{\mathrm{n}}-P t_{0}, \bar{u}_{\mathrm{n}}-\bar{\psi}-\operatorname{ess} \sup \left(\bar{u}_{\mathrm{n}}-\bar{\psi}\right)\right\rangle_{H^{-1 / 2}, H^{1 / 2}}=0 .
$$

Therefore:

$$
\forall \hat{u} \in K_{0}, \quad\left\langle\tilde{t}_{\mathrm{n}}-P t_{0}, \hat{u}-\bar{\psi}-\operatorname{ess} \sup (\hat{u}-\bar{\psi})\right\rangle_{H^{-1 / 2}, H^{1 / 2}} \geq 0,
$$


and, since $\tilde{t}_{\mathrm{n}} \in H_{0}^{\prime}, P>0$ :

$\forall \hat{u} \in K_{0}, \quad \forall C \in \mathbb{R}^{+} \quad\left\langle\tilde{t}_{\mathrm{n}}-P t_{0}, \hat{u}-\bar{\psi}-\operatorname{ess} \sup (\hat{u}-\bar{\psi})-C\right\rangle_{H^{-1 / 2}, H^{1 / 2}} \geq 0$,

which can be summarized as:

$$
\forall \hat{u} \in H^{1 / 2}(-1,1) \text { such that: } \hat{u} \leq 0, \quad\left\langle\tilde{t}_{\mathrm{n}}-P t_{0}, \hat{u}\right\rangle_{H^{-1 / 2}, H^{1 / 2}} \geq 0 .
$$

This is nothing but $\tilde{t}_{\mathrm{n}}-P t_{0} \leq 0$. Finally, take an arbitrary function $\rho \in$ $\mathscr{D}(]-1,1[)$. The function:

$$
\hat{u}=\bar{\psi}+\left((\max \rho) \chi_{]-1,1[}-\rho\right)\left(\bar{u}_{\mathrm{n}}-\bar{\psi}-\operatorname{ess} \sup \left(\bar{u}_{\mathrm{n}}-\bar{\psi}\right)\right),
$$

belongs to $K_{0}$ and statement (ii) entails:

$$
\left\langle\tilde{t}_{\mathrm{n}}-P t_{0},\left(\bar{u}_{\mathrm{n}}-\bar{\psi}-\operatorname{ess} \sup \left(\bar{u}_{\mathrm{n}}-\bar{\psi}\right)\right) \rho\right\rangle_{H^{-1 / 2}, H^{1 / 2}} \leq 0
$$

and since this is true also for $-\rho$, this inequality is actually an identity. The last statement in the contact condition (11) is therefore proved.

Remark. Since $\varphi$ is a proper, convex, lower semicontinuous function on $H_{0}$

(lemma 4), statement (ii) in proposition 5 can be expressed in the language of subdifferentials as:

$$
-\tilde{t}_{\mathrm{n}} \in \partial \varphi\left[\bar{u}_{\mathrm{n}}\right] .
$$

Similarly, setting:

$$
\varphi^{*}(-\hat{t}) \stackrel{\text { def }}{=}-\langle\bar{\psi}, \hat{t}\rangle_{H_{0}, H_{0}^{\prime}}+I_{K_{0}^{\prime}}(\hat{t}),
$$

(where $I_{S}$ denotes the indicator function of $S$, that is, taking the value 0 on $S$ and $+\infty$ outside), statement (i) reads as:

$$
\bar{u}_{\mathrm{n}} \in \partial \varphi^{*}\left[-\tilde{t}_{\mathrm{n}}\right],
$$

405 showing that $\varphi$ is nothing but the Legendre-Fenchel conjugate of $\varphi^{*}$ (up to some additive constant), and reciprocally.

Making use of the above definition and results, we have the following weak formulation for the formal problem (3) governing the normal component $t_{\mathrm{n}}$ (or equivalently $\tilde{t}_{\mathrm{n}}$ ) of the surface traction exerted by the moving obstacle.

Problem $\mathbf{I}^{\prime}$. Let $f \in \mathbb{R}, P$ be positive and $\bar{\psi} \in H_{0}$. Find $\tilde{t}_{\mathrm{n}} \in K_{0}^{\prime}$ such that:

$$
\forall \hat{t} \in K_{0}^{\prime}, \quad b\left(\tilde{t}_{\mathrm{n}}, \hat{t}-\tilde{t}_{\mathrm{n}}\right) \geq\left\langle\bar{\psi}, \hat{t}-\tilde{t}_{\mathrm{n}}\right\rangle_{H_{0}, H_{0}^{\prime}} .
$$

Straightforwardly applying the Lions-Stampacchia theorem (see [1] or theorem 25 of which the Lions-Stampacchia theorem is a particular case) gives: 
Proposition 6. Problem $I^{\prime}$ has a unique solution.

To obtain the dual formulation of problem $\mathrm{I}^{\prime}$, we first construct the dual

Problem I. Let $f \in \mathbb{R}, P$ be positive and $\bar{\psi} \in H_{0}$. Find $\bar{u}_{\mathrm{n}} \in K_{0}$ such that:

$$
\forall \hat{u} \in K_{0}, \quad a\left(\hat{u}-\bar{u}_{\mathrm{n}}, \bar{u}_{\mathrm{n}}\right)+\varphi(\hat{u})-\varphi\left(\bar{u}_{\mathrm{n}}\right) \geq 0,
$$

where $t_{0}(x)$ is the function defined by formula (8).

Proposition 7. Problem I has a unique solution. The unique solutions $\tilde{t}_{\mathrm{n}}$ and $\bar{u}_{\mathrm{n}}$ of problems $I^{\prime}$ and $I$ are linked by the relationship:

$$
\tilde{t}_{\mathrm{n}}=\mathscr{L}\left(\bar{u}_{\mathrm{n}}\right) .
$$

Proof. Note that the mapping $\hat{u} \mapsto\left\langle\operatorname{ess} \sup (\hat{u})-\hat{u}, P t_{0}\right\rangle$ is not linear, so that of problem I. However, the extension of the Lions-Stampacchia theorem that is provided in appendix A (theorem 25) together with lemma 4 yields a unique solution of problem I. Another proof can also be proposed. Indeed, taking $\tilde{t}_{\mathrm{n}}$ the unique solution of problem $\mathrm{I}^{\prime}$ provided by proposition 6 and setting:

$$
\bar{u}_{\mathrm{n}}=\mathscr{L}^{-1}\left(\tilde{t}_{\mathrm{n}}\right),
$$

430 we get a solution of problem I, thanks to proposition 5. This solution is the only one, thanks to the coercivity of the bilinear form $a$.

Summing up, denoting by pv $1 / x$ the distributional derivative of the locally integrable function $\log |x|$, we have proved the following theorem.

Theorem 8. Let $f \in \mathbb{R}, P$ be positive and $\bar{\psi} \in H^{1 / 2}(-1,1)$. There exists a 435 unique $\left(\bar{u}_{\mathrm{n}}, t_{\mathrm{n}}\right) \in H^{1 / 2}(-1,1) \times H^{-1 / 2}(-1,1)$ such that:

$$
\begin{aligned}
& \left.-\frac{1}{\pi} t_{\mathrm{n}} * \mathrm{pv} 1 / x+\gamma f t_{\mathrm{n}}=\bar{u}_{\mathrm{n}}^{\prime}, \quad \text { in }\right]-1,1[, \\
& \bar{u}_{\mathrm{n}}-\bar{\psi} \leq 0, \quad t_{\mathrm{n}} \leq 0, \quad\left(\bar{u}_{\mathrm{n}}-\bar{\psi}\right) t_{\mathrm{n}}=0, \\
& \left\langle t_{\mathrm{n}}, \chi_{]-1,1[}\right\rangle_{H^{-1 / 2}, H^{1 / 2}}=-P .
\end{aligned}
$$

Finally, if $\bar{\psi}$ is replaced by $\bar{\psi}+C(C \in \mathbb{R})$, then the corresponding solution is $\left(\bar{u}_{\mathrm{n}}+C, t_{\mathrm{n}}\right)$. 
To conclude this section about homogeneous friction, we establish the form of the universal singularities that should be expected in the solutions of problems I and $\mathrm{I}^{\prime}$, in the spirit of the singularities of the solutions of linear elasticity in polyhedra, as discussed by Grisvard [9].

The following theorem shows that a logarithmic singularity must be expected at a wedge of the moving indentor.

Theorem 9. Assume that $\bar{\psi}^{\prime}$ is piecewise Lipschitz-continuous. If the unique ${ }_{445}$ solution of the problem in theorem 8 is such that contact is achieved in a neighbourhood of $c \in]-1,1[$, then:

$$
t_{\mathrm{n}}=\frac{\bar{\psi}^{\prime}(c+)-\bar{\psi}^{\prime}(c-)}{1+(\gamma f)^{2}}\left(\frac{\log |x-c|}{\pi}+\gamma f H(x-c)\right)+\theta,
$$

where $H$ is the Heaviside function and $\theta$ is a measure whose restriction to a neighbourhood of $c$ is a continuous function.

Proof. Set:

$$
\bar{\phi}(x)=\bar{\psi}^{\prime}(c-)(x-c) \chi_{]-1, c[}(x)+\bar{\psi}^{\prime}(c+)(x-c) \chi_{] c, 1[}(x),
$$

where $\chi_{S}$ is the indicator function of the set $S$. We have the decomposition:

$$
t_{\mathrm{n}}=\mathscr{L}(\bar{\phi})+\mathscr{L}(\bar{\psi}-\bar{\phi})+\mathscr{L}\left(\bar{u}_{\mathrm{n}}-\bar{\psi}\right)-P t_{0}
$$

Since there exists $\epsilon>0$ such that $] c-\epsilon, c+\epsilon$ [ does not meet the support of $\bar{u}_{\mathrm{n}}-\bar{\psi}$, the restriction of $\mathscr{L}\left(\bar{u}_{\mathrm{n}}-\bar{\psi}\right)$ to $] c-\epsilon, c+\epsilon\left[\right.$ is a $C^{\infty}$ function. Besides, the function $\bar{\psi}^{\prime}-\bar{\phi}^{\prime}$ is Lipschitz-continuous in a neighbourhood of $c$ which entails that the function $\mathscr{L}(\bar{\psi}-\bar{\phi})$ is continuous in a neighbourhood of $c$, thanks to 455 formula (10) and proposition 7 of [5]. Therefore:

$$
t_{\mathrm{n}}=\mathscr{L}(\bar{\phi})+\tilde{\theta}
$$

for some measure $\tilde{\theta}$ whose restriction to a neighbourhood of $c$ is a continuous function. The claim in the theorem is now a straightforward consequence of formula (10) and propositions 7 and 8 of [5].

Remark. Since $t_{\mathrm{n}}$ is a nonpositive measure, proposition 9 entails in particular that, if active contact is achieved around a wedge of the indentor, then this wedge must satisfy:

$$
\bar{\psi}^{\prime}(c+) \geq \bar{\psi}^{\prime}(c-),
$$

otherwise (reentrant corner of the obstacle), it is impossible for active contact to occur all over a neighbourhood of $c$.

The following theorem describes the singularities that should be expected at 465 the boundary of the contact zone and shows in particular that the front and rear power singularities encountered at the edges of the rigid flat punch are universal. 
Theorem 10. Assume that $\bar{\psi}^{\prime}$ is piecewise Lipschitz-continuous. If the unique solution of problem $I$ is such that contact is achieved in a right-neighbourhood of $c \in\left[-1,1\left[\right.\right.$, that is, $\bar{u}_{\mathrm{n}}=\bar{\psi}$ in $] c, c+\epsilon[$, then the following estimates at $c$ hold true.

- In the case where $c=-1$, we have:

$$
t_{\mathrm{n}}=-\frac{\theta_{-}}{(1+x)^{1 / 2+\alpha}},
$$

where

$$
\alpha \stackrel{\text { def }}{=} \frac{1}{\pi} \arctan (\gamma f) \quad(\in]-1 / 2,1 / 2[),
$$

and $\theta_{-}$is a measure whose restriction to a right-neighbourhood of $x=-1$ is a Hölder-continuous function. In the case where $\theta_{-}(-1)>0$, then the following estimate holds true:

$$
\bar{u}_{\mathrm{n}}^{\prime}(x) \sim-\frac{\theta_{-}(-1) \sqrt{1+\gamma^{2} f^{2}}}{|1+x|^{1 / 2+\alpha}}, \quad \text { as } x \rightarrow-1-.
$$

- In the case where $c>-1$ and detachment occurs in a left-neighbourhood of $c$, that is $\bar{u}_{\mathrm{n}}<\bar{\psi}$ in $] c-\epsilon, c[$, we have:

$$
t_{\mathrm{n}}=-|c-x|^{1 / 2-\alpha} \theta_{-}
$$

where $\theta_{-}$is a measure whose restriction to a right-neighbourhood of $x=c$ is a Hölder-continuous function.

480

Similarly, if the unique solution of problem I is such that contact is achieved in a left-neighbourhood of $c \in]-1,1]$, that is, $\bar{u}_{\mathrm{n}}=\bar{\psi}$ in $] c-\epsilon, c[$, then the following estimates at c hold true.

- In the case where $c=1$, we have:

$$
t_{\mathrm{n}}=-\frac{\theta_{+}}{(1-x)^{1 / 2-\alpha}},
$$

where $\theta_{+}$is a measure whose restriction to a left-neighbourhood of $x=1$ is a Hölder-continuous function. In the case where $\theta_{+}(1)>0$, then the following estimate holds true:

$$
\bar{u}_{\mathrm{n}}^{\prime}(x) \sim \frac{\theta_{+}(1) \sqrt{1+\gamma^{2} f^{2}}}{|1-x|^{1 / 2-\alpha}}, \quad \text { as } x \rightarrow 1+.
$$

- In the case where $c<1$ and detachment occurs in a right-neighborhood of $c$, that is $\bar{u}_{\mathrm{n}}<\bar{\psi}$ in $] c, c+\epsilon[$, we have:

$$
t_{\mathrm{n}}=-|x-c|^{1 / 2+\alpha} \theta_{+}
$$

where $\theta_{+}$is a measure whose restriction to a left-neighbourhood of $x=c$ is a Hölder-continuous function. 
Proof. The proof will be developed only for the case where contact occurs in a right-neighbourhood of $c$. We first consider the case where $c=-1$. Set:

$$
\bar{\phi}(x) \equiv(x+1) \bar{\psi}^{\prime}(-1+) .
$$

We have the decomposition:

$$
t_{\mathrm{n}}=\mathscr{L}(\bar{\phi})+\mathscr{L}(\bar{\psi}-\bar{\phi})+\mathscr{L}\left(\bar{u}_{\mathrm{n}}-\bar{\psi}\right)-P t_{0}
$$

Since, $\bar{u}_{\mathrm{n}}-\bar{\psi}$ is supported in $[-1+\epsilon, 1]$, the restriction to $]-1,-1+\epsilon[$ of the term $\mathscr{L}\left(\bar{u}_{\mathrm{n}}-\bar{\psi}\right)$ is readily seen to equal the product of $t_{0}$ with a $C^{\infty}$ function. Therefore, the claim in the proposition holds true for that term. The term $\mathscr{L}(\bar{\psi}-\bar{\phi})$ can be developed by means of formula (10). The Cauchy integral in that expression is seen to be the product of $t_{0}$ with some Cauchy integral of the form:

$$
\oint_{-1}^{1} \frac{\left(1+x^{\prime}\right)^{1+\beta} \eta\left(x^{\prime}\right)}{x^{\prime}-x} \mathrm{~d} x^{\prime}
$$

for some positive $\beta$ and some Hölder-continuous function $\eta$. Splitting

$$
\left(1+x^{\prime}\right)^{1+\beta}=(1+x)\left(1+x^{\prime}\right)^{\beta}+\left(x^{\prime}-x\right)\left(1+x^{\prime}\right)^{\beta},
$$

it entails:

$$
\mathscr{L}(\bar{\psi}-\bar{\phi})=\left(a^{\prime}+(1+x) \tilde{\theta}\right) t_{0},
$$

for some constant $a^{\prime} \in \mathbb{R}$ and some Hölder-continuous function $\tilde{\theta}$, thanks to proposition 7 of [5]. Besides, the term $\mathscr{L}(\bar{\phi})$ is:

$$
\begin{aligned}
\mathscr{L}(\bar{\phi}) & =\frac{\bar{\psi}^{\prime}(-1+)}{1+(\gamma f)^{2}}\left\{\gamma f-\frac{1}{\pi} \oint_{-1}^{1} \frac{\left(1+x^{\prime}\right)^{\frac{1}{2}+\alpha}\left(1-x^{\prime}\right)^{\frac{1}{2}-\alpha}}{(1+x)^{\frac{1}{2}+\alpha}(1-x)^{\frac{1}{2}-\alpha}} \cdot \frac{\mathrm{d} x^{\prime}}{x^{\prime}-x}\right\} \\
& =\left(a^{\prime \prime}+b^{\prime \prime}(1+x)\right) t_{0}(x)
\end{aligned}
$$

thanks to formula (19) in [5]. Finally, it has been proved a little bit more than claimed:

$$
t_{\mathrm{n}}=-\frac{a+(1+x) \theta}{(1+x)^{1 / 2+\alpha}}
$$

for some constant $a \in \mathbb{R}$ and some measure $\theta$ whose restriction to a rightneighbourhood of $c=-1$ is a Hölder-continuous function. This stronger result will be of use to prove the claim in the case $c>-1$. Since, for $x<-1$ :

$$
\bar{u}_{\mathrm{n}}^{\prime}(x)=\frac{1}{\pi} \int_{-1}^{1} \frac{t_{\mathrm{n}}\left(x^{\prime}\right)}{x^{\prime}-x},
$$

the estimate for $\bar{u}_{\mathrm{n}}^{\prime}(x)$ is now a straightforward consequence of proposition 8 in [5], and:

$$
\sin \pi(1 / 2+\alpha)=\frac{1}{\sqrt{1+\gamma^{2} f^{2}}} .
$$


It remains to prove the claim for the case $c>-1$. By hypothesis, $t_{\mathrm{n}}$ vanishes on $] c-\epsilon, c\left[\right.$ (taking $\epsilon$ smaller if necessary). We denote by $\tilde{t}_{\mathrm{n}}$ the restriction of $t_{\mathrm{n}}$ to $]-1, c-\epsilon\left[\right.$. Then, we denote by $\tilde{u}_{\mathrm{n}} \in C^{\infty}([c, 1])$, the function defined by:

$$
\tilde{u}_{\mathrm{n}}(x)=\frac{1}{\pi} \int_{-1}^{c-\epsilon} \frac{\tilde{t}_{\mathrm{n}}\left(x^{\prime}\right)}{x^{\prime}-x},
$$

so that the function $\bar{u}_{\mathrm{n}}-\tilde{u}_{\mathrm{n}}$ defined on $[c, 1]$ is the unique solution of the problem I where $\bar{\psi}$ is replaced by $\bar{\psi}-\tilde{u}_{\mathrm{n}}, P$ is replaced by $P+\int_{-1}^{c-\epsilon} \tilde{t}_{\mathrm{n}}$ and $]-1,1[$ is replaced by $] c, 1[$. Hence, $c$ is the left edge for this new contact problem and the results of the first part of the proof can be applied, which gives, for $x \in] c, 1[$ :

$$
t_{\mathrm{n}}=-\frac{a+(x-c) \theta}{(x-c)^{1 / 2+\alpha}}
$$

for some constant $a \in \mathbb{R}$ and some measure $\theta$ whose restriction to a rightneighbourhood of $c$ is a Hölder-continuous function. We must have $a \geq 0$. If $a \neq 0$, then:

$$
\bar{u}_{\mathrm{n}}^{\prime}(x) \sim-\frac{a \sqrt{1+\gamma^{2} f^{2}}}{(c-x)^{1 / 2+\alpha}}, \quad \text { as } x \rightarrow c-.
$$

But this is incompatible with $\bar{u}_{\mathrm{n}}-\bar{\psi} \leq 0$ on $]-1,1[$. Therefore $a=0$ and the 520 expected claim is proved.

\section{Analysis of the case where $f(x)=f_{-} \chi_{]-1,0[}(x)+f_{+} \chi_{] 0,1[}(x)$}

In this section, we focus on the case where the friction coefficient has only one jump located at $x=0$. The complete understanding of that situation will provide all the necessary tools to handle the more general situation where the friction coefficient $f(x)$ is an arbitrary step function (synonymously, piecewise constant function). From now on, we set:

$$
s(x) \stackrel{\text { def }}{=} \gamma f(x)
$$

so that the steady sliding frictional contact problem to solve now reads formally as:

- $\frac{1}{\pi} \oint_{-1}^{1} \frac{t_{\mathrm{n}}(t)}{t-x} \mathrm{~d} t+s(x) t_{\mathrm{n}}(x)=\bar{u}_{\mathrm{n}}^{\prime}(x)$,

- $\exists C \in \mathbb{R}, \quad \bar{u}_{\mathrm{n}}+C \leq \bar{\psi}, \quad t_{\mathrm{n}} \leq 0, \quad\left(\bar{u}_{\mathrm{n}}+C-\bar{\psi}\right) t_{\mathrm{n}} \equiv 0$,

- $\int_{-1}^{1} t_{\mathrm{n}}(t) \mathrm{d} t=-P$

and we focus on the case where:

$$
s(x)=\mid \begin{array}{ll}
s_{-} & \text {if } x \in]-1,0[, \\
s_{+} & \text {if } x \in] 0,1[,
\end{array}
$$


for two real constants $s_{-}, s_{+} \in \mathbb{R}$. Since the product $s \hat{t}$ is generally undefined for $\hat{t} \in H^{-1 / 2}(-1,1)$, an appropriate functional framework must be designed.

This is the object of the next section.

\subsection{A functional framework}

To be able to formulate the corresponding problem, we introduce the space $\bar{H}^{\prime}$ defined as the completion of $L^{2}(-1,1)$ (or $\left.C^{\infty}([-1,1])\right)$ in the norm:

$$
\|\varphi\|_{\bar{H}^{\prime}} \stackrel{\text { def }}{=}\left(\|\varphi\|_{H^{-1 / 2}}^{2}+\left(\int_{0}^{1} \varphi\right)^{2}\right)^{1 / 2} .
$$

It is readily checked that $\bar{H}^{\prime}$ is a Hilbert space which is contained in $H^{-1 / 2}$, and that the two linear forms defined on $C^{\infty}([-1,1])$ by:

$$
\varphi \mapsto \int_{-1}^{0} \varphi, \quad \varphi \mapsto \int_{0}^{1} \varphi
$$

extend into continuous linear forms over $\bar{H}^{\prime}$ (this last result is not true if $\bar{H}^{\prime}$ is replaced with $H^{-1 / 2}$ ). In the sequel, we will commit the abuse of keeping the above integral notation for general $\varphi \in \bar{H}^{\prime}$. The dual space $\bar{H}$ of $\bar{H}^{\prime}$ is:

$$
\bar{H}=\left\{u+C \chi_{] 0,1[} \mid u \in H^{1 / 2}(-1,1) \text { and } C \in \mathbb{R}\right\},
$$

where $\chi_{] 0,1[}$ is the function that takes identically the value 1 on $] 0,1[$ and 0 outside.

Proposition 11. The following embeddings are continuous:

$$
H^{-1 / 2}(-1,0) \times H^{-1 / 2}(0,1) \subset \bar{H}^{\prime} \subset H^{-1 / 2}(-1,1) .
$$

In addition, these three spaces are distinct.

Proof. The restriction operator from $H^{1 / 2}(-1,1)$ into $H^{1 / 2}(0,1)$ is obviously continuous. Going to the dual side, it gives the existence of a real constant $C$ such that:

$$
\begin{aligned}
& \forall \varphi \in H^{-1 / 2}(-1,0) \times H^{-1 / 2}(0,1), \\
& \qquad \varphi \varphi\left\|_{H^{-1 / 2}(-1,1)} \leq C\right\| \varphi \|_{H^{-1 / 2}(-1,0) \times H^{-1 / 2}(0,1)} .
\end{aligned}
$$

The continuity of the second embedding is trivial and that of the first one is easily deduced from the latter estimate.

For all $C \in \mathbb{R}$, the function $C+\log |\log | x / 2||$ is in $H^{1 / 2}(-1,1)$ (thanks to the trace theorem and the fact that $C+\log |\log | \sqrt{x^{2}+y^{2}} / 2||$ is in $H^{1}$ of the unit disk). However, based on theorem 31, the function defined by:

$$
\mid \begin{array}{ll}
C+\log |\log | x / 2||, & \text { if } x \in]-1,0[, \\
0, & \text { if } x \in] 0,1[,
\end{array}
$$


is not in $H^{1 / 2}(-1,1)$. Therefore, the function:

$$
\begin{array}{|ll}
\log |\log | x / 2||, & \text { if } x \in]-1,0[, \\
0, & \text { if } x \in] 0,1[,
\end{array}
$$

is not in $\bar{H}$, whereas it is obviously in $H^{1 / 2}(-1,0) \times H^{1 / 2}(0,1)$. These two 550 spaces are therefore distinct and the same is true of their dual spaces $\bar{H}^{\prime}$ and $H^{-1 / 2}(-1,0) \times H^{-1 / 2}(0,1)$. For the second embedding, the function $u$ defined by:

$$
u(x)=\mid \begin{array}{ll}
\log |\log | x / 2||-\log |\log | 1 / 2||, & \text { if } x \in]-1,1[, \\
0, & \text { if } x \notin]-1,1[,
\end{array}
$$

is in $H^{1 / 2}(\mathbb{R})$. The sequence $\left(u_{n}\right)$ defined by:

$$
u_{n}(x)=\mid \begin{array}{ll}
0, & \text { if } x \notin]-1,1[, \\
\log |\log | 1 /(2(n+1))||-\log |\log | 1 / 2||, & \text { if } x \in]-\frac{1}{(n+1)}, \frac{1}{(n+1)}[, \\
\log |\log | x / 2||-\log |\log | 1 / 2||, & \text { otherwise, }
\end{array}
$$

converges towards $u$ in $H^{1 / 2}(\mathbb{R})$. Therefore, the sequence $\left(u_{n}^{\prime}\right)$ converges towards $555 u^{\prime}$ in $H^{-1 / 2}(\mathbb{R})$. Since all the $u_{n}^{\prime}$ are supported in $[-1,1]$, the sequence of the restrictions to $[-1,1]$ of each $u_{n}^{\prime}$ converges in $H^{-1 / 2}(-1,1)$ towards the restriction of $u^{\prime}$. However:

$$
\int_{0}^{1} u_{n}^{\prime}=\log |\log | 1 / 2||-\log |\log | 1 /(2(n+1))||,
$$

is unbounded. Finally, $u^{\prime}$ is in $H^{-1 / 2}(-1,1)$, but not in $\bar{H}^{\prime}$.

Theorem 12. We denote by $H_{00}^{1 / 2}(] 0,1[)$ the Hilbert space (see theorem 31) of all the functions in $H^{1 / 2}(] 0,1[)$ such that the extension by zero is in $H^{1 / 2}(]-1,1[)$. Let $t \in \bar{H}^{\prime}$ be arbitrary. We consider, first, the restriction $t_{\mid] 0,1[}$ of $t$ to ]0,1[ and then, the extension $\bar{t}$ of this restriction, by zero to the whole real line $\mathbb{R}$. Then, $\bar{t}$ is a distribution on $\mathbb{R}$ (such a result fails to be true for $t \in H^{-1 / 2}(-1,1)$ in general). Therefore, denoting by $H(\cdot)$ the Heaviside function, the convolution 565 product $H * \bar{t}$ is well-defined in the sense of Schwartz's distributions. In addition, the restriction of $H * \bar{t}$ to the interval $] 0,1\left[\right.$ is in $H_{00}^{1 / 2}(] 0,1[)$, and the continuity estimate:

$$
\forall t_{1}, t_{2} \in \bar{H}^{\prime}, \quad\left\langle\bar{t}_{1}, H * \bar{t}_{2}\right\rangle \leq M\left\|t_{1}\right\|_{\bar{H}^{\prime}}\left\|t_{2}\right\|_{\bar{H}^{\prime}},
$$

holds true for some real constant $M$ independent of $t_{1}$ and $t_{2}$.

Proof. Let $t \in \bar{H}^{\prime}$ and $\varphi \in C_{\mathrm{c}}^{\infty}(\mathbb{R})$ be arbitrary. The restriction of $\varphi(\cdot)-\varphi(0)$ to $] 0,1\left[\right.$ is in $H_{00}^{1 / 2}(] 0,1[)$ and we therefore have:

$$
\begin{aligned}
\langle\bar{t}, \varphi-\varphi(0)\rangle & \leq C\|\varphi-\varphi(0)\|_{H_{00}^{1 / 2}(] 0,1[)}, \\
& \leq C^{\prime}\|\varphi-\varphi(0)\|_{C^{1}([-1,1])}
\end{aligned}
$$


for some constants $C, C^{\prime} \in \mathbb{R}$. Since:

$$
\langle\bar{t}, \varphi(0)\rangle=\left\langle\bar{t}, \varphi(0) \chi_{0,1[}\right\rangle=\varphi(0) \int_{0}^{1} \bar{t}=\varphi(0) \int_{0}^{1} t,
$$

570 it is readily seen that $\bar{t}$ defines a Schwartz distribution (of order 1 ) on $\mathbb{R}$. Note that if $t$ is only in $H^{-1 / 2}(-1,1)$ (instead of $\bar{H}^{\prime}$ ), then the restriction $t_{\mid] 0,1[}$ of $t$ to ]0, 1 [ is only in $H_{00}^{1 / 2^{\prime}}(] 0,1[)$ which does not make it possible to define $\left\langle t_{\mid] 0,1[}, \chi_{] 0,1[}\right\rangle$ in general.

Now, take $t \in \bar{H}^{\prime}$ arbitrary and $\varphi_{n}$ a sequence in $C_{\mathrm{c}}^{\infty}(]-1,1[)$ that converges strongly towards $t$ in $\bar{H}^{\prime}$. The sequence $H * \varphi_{n}=\int_{-1}^{x} \varphi_{n}$ converges towards $H * t$ in the sense of distributions. Similarly, $H * \bar{\varphi}_{n}$ converges towards $H * \bar{t}$ in the sense of distributions. Thanks to the use of the Fourier transform, the continuity property:

$$
\forall t \in H^{-1 / 2}(-1,1), \quad\|H * t\|_{H^{1 / 2}(-1,1)} \leq M\|t\|_{H^{-1 / 2}(-1,1)},
$$

holds true, for some real constant independent of $t$. But, $H * \bar{\varphi}_{n}$ is nothing but the $C^{\infty}$ function equalling 0 on $]-1,0\left[\right.$ and $H * \varphi_{n}-\int_{-1}^{0} \varphi_{n}$ on $] 0,1[$. Therefore,

$$
\left\|H * \bar{\varphi}_{n}\right\|_{H^{1 / 2}(-1,1)} \leq M\left\|\varphi_{n}\right\|_{H^{-1 / 2}(-1,1)}+\left|\int_{-1}^{0} \varphi_{n}\right| \leq M^{\prime}\left\|\varphi_{n}\right\|_{\bar{H}^{\prime}},
$$

for some constant $M^{\prime}$ independent of $n$. Hence, $H * \bar{\varphi}_{n}$ converges to some function in $H^{1 / 2}(-1,1)$ which is supported in [0,1[. This limit must equal $H * \bar{t}$. We therefore have proved:

$$
\forall t \in \bar{H}^{\prime}, \quad\|H * \bar{t}\|_{H_{00}^{1 / 2}(0,1)} \leq M\|t\|_{\bar{H}^{\prime}},
$$

for some (other) real constant $M$ independent of $t$. This is enough to get the whole claim in the theorem.

Theorem 13. The bilinear mapping defined for $\varphi_{1}, \varphi_{2} \in C^{\infty}([-1,1])$ by:

$$
\left(\varphi_{1}, \varphi_{2}\right) \mapsto \int_{-1}^{1} \varphi_{1}(x) \int_{-1}^{x} s\left(x^{\prime}\right) \varphi_{2}\left(x^{\prime}\right) \mathrm{d} x^{\prime} \mathrm{d} x,
$$

defines a continuous bilinear mapping on $\bar{H}^{\prime} \times \bar{H}^{\prime}$.

\section{Proof.}

Step 1. Case where $s(x)$ is constant.

Let us note that in the particular case where $s(x)$ is constant, the linear mapping under consideration is nothing but:

$$
s\left\langle\varphi_{1}, \varphi_{2} * H\right\rangle_{H^{-1 / 2}(-1,1), H^{1 / 2}(-1,1)}
$$


where $H(x)$ denotes the Heaviside function and $\varphi_{2} * H$ denotes the convolution product of $H$ with the extension by 0 on the whole line of $\varphi_{2}$. The extension of an arbitrary distribution in $H^{-1 / 2}(-1,1)$ by 0 outside $]-1,1\left[\right.$ is in $H^{-1 / 2}(\mathbb{R})$. The convolution with $H(x)$ is in $H_{\text {loc }}^{1 / 2}(\mathbb{R})$ (because its derivative is in $H^{-1 / 2}(\mathbb{R})$ ) and the restriction of this convolution product to ]-1, 1[ is therefore in $H^{1 / 2}(]-1,1[)$. This bilinear mapping is therefore continuous on $H^{-1 / 2}(-1,1) \times H^{-1 / 2}(-1,1)$. By proposition 11, it is therefore continuous on $\bar{H}^{\prime} \times \bar{H}^{\prime}$. Hence, it is now sufficient to prove theorem 13 in the particular case where $s_{-}=0$ and $s_{+}=1$ which will be assumed from now on.

Step 2. Case where $s_{-}=0$ and $s_{+}=1$.

Take $t_{1}, t_{2} \in \bar{H}^{\prime}$. With the same notations as the ones in theorem 12, the bilinear form under consideration is nothing but:

$$
\left\langle\bar{t}_{1}, H * \bar{t}_{2}\right\rangle \leq M\left\|t_{1}\right\|_{\bar{H}^{\prime}}\left\|t_{2}\right\|_{\bar{H}^{\prime}},
$$

which is well-defined and continuous, thanks to theorem 12 .

Theorem 13 shows that the bilinear form:

$$
\bar{b}\left(t_{1}, t_{2}\right) \stackrel{\text { def }}{=}-\frac{1}{\pi}\left\langle t_{1} * \log |x|, t_{2}\right\rangle_{H^{1 / 2}, H^{-1 / 2}}+\left\langle\int_{-1}^{x} s t_{1}, t_{2}\right\rangle_{\bar{H}, \bar{H}^{\prime}},
$$

is continuous on $\bar{H}^{\prime}$. As previously, we focus on the closed subspace $\bar{H}_{0}^{\prime}$ of $\bar{H}^{\prime}$ defined by:

$$
\bar{H}_{0}^{\prime} \stackrel{\text { def }}{=}\left\{\hat{t} \in \bar{H}^{\prime} \mid\left\langle\hat{t}, \chi_{]-1,1[}\right\rangle_{\bar{H}^{\prime}, \bar{H}}=0\right\} .
$$

In order to analyse the coercivity of $\bar{b}$ on $\bar{H}_{0}^{\prime}$, we are led to perform the following calculation, for arbitrary $p \in C^{\infty}([-1,1])$ :

$$
\begin{gathered}
-\frac{1}{\pi} \int_{-1}^{1} \int_{-1}^{1} p(x) p(t) \log |x-t| \mathrm{d} t \mathrm{~d} x+\int_{-1}^{1} \int_{-1}^{x} s(t) p(t) p(x) \mathrm{d} t \mathrm{~d} x+ \\
C\left(\int_{-1}^{1} s p\right)\left(\int_{-1}^{1} p\right)=-\frac{1}{\pi} \int_{-1}^{1} \int_{-1}^{1} p(x) p(t) \log |x-t| \mathrm{d} t \mathrm{~d} x+\frac{s_{+}-s_{-}}{2}\left(\int_{0}^{1} p\right)^{2} \\
\quad+\frac{s_{-}(1+2 C)}{2}\left(\int_{-1}^{1} p\right)^{2}+\left(s_{+}-s_{-}\right) C\left(\int_{-1}^{1} p\right)\left(\int_{0}^{1} p\right)
\end{gathered}
$$

A surprising fact happens: under the condition that $s_{+}>s_{-}$, the bilinear form is coercive on $\bar{H}_{0}^{\prime}$, but it is not even nonnegative on $\bar{H}_{0}^{\prime}$ in the case ${ }_{610} s_{+}<s_{-}$(otherwise, one would have $\bar{H}^{\prime}=H^{-1 / 2}$, which is not true thanks to proposition 11). We must therefore study these two cases separately.

\subsection{Analysis of the case $s_{+}>s_{-}$}

Based on theorem 36 in appendix $\mathrm{C}$, the function

$$
t_{0}(x)=\frac{e^{\tau(x)}}{\pi(1-x) \sqrt{1+s^{2}(x)}},
$$


where:

$$
\tau(x)=\frac{1}{\pi} \oint_{-1}^{1} \arctan \left(-1 / s\left[x^{\prime}\right)\right) \frac{\mathrm{d} x^{\prime}}{x^{\prime}-x},
$$

${ }_{615}$ is in $L^{p}(-1,1)$ for some $p>1$, is positive and satisfies:

$$
\forall \hat{t} \in \bar{H}_{0}^{\prime}, \quad \bar{b}\left(t_{0}, \hat{t}\right)=0 .
$$

In addition,

$$
\int_{-1}^{1} t_{0}\left(x^{\prime}\right) \mathrm{d} x^{\prime}=1
$$

thanks to the identity proved in the step 1 of the proof of theorem 14 of [5] (used with the choices $\lambda=-1$ and $x=1$ ). As previously, the function $t_{0}$ is going to be used to make a shift of the unknown in the steady sliding frictional contact ${ }_{620}$ problem. We set:

$$
\bar{K}_{0}^{\prime} \stackrel{\text { def }}{=}\left\{\hat{t} \in \bar{H}_{0}^{\prime} \mid \hat{t}-P t_{0} \leq 0\right\},
$$

Since $t_{0}$ is positive, $\bar{K}_{0}^{\prime}$ is a nonempty closed convex subset of $\bar{H}_{0}^{\prime}$. The dual space $\bar{H}_{0}$ of $\bar{H}_{0}^{\prime}$ is the quotient space $\bar{H} / \mathscr{C}$ (where $\mathscr{C}$ is the line of all the constant functions on $]-1,1[$ ).

Making the shift of unknown:

$$
t_{\mathrm{n}}=\tilde{t}_{\mathrm{n}}-P t_{0},
$$

the steady sliding frictional contact problem now reads as follows.

Problem II'. Let $\bar{\psi} \in \bar{H}_{0} \supset H^{1 / 2}(-1,1) / \mathscr{C}$. Find $\tilde{t}_{\mathrm{n}} \in \bar{K}_{0}^{\prime}$ such that:

$$
\forall \hat{t} \in \bar{K}_{0}^{\prime}, \quad \bar{b}\left(\tilde{t}_{\mathrm{n}}, \hat{t}-\tilde{t}_{\mathrm{n}}\right) \geq\left\langle\bar{\psi}, \hat{t}-\tilde{t}_{\mathrm{n}}\right\rangle_{\bar{H}_{0}, \bar{H}_{0}^{\prime}} .
$$

Since in the case $s_{+}>s_{-}, \bar{b}$ is continuous and coercive on $\bar{H}_{0}^{\prime}$, we have already proved the

Proposition 14. Assume that $s_{+}>s_{-}$, then problem II' has a unique solution.

For arbitrary $u \in \bar{H}_{0}$, there exists a unique $\tilde{t} \in \bar{H}_{0}^{\prime}$ such that:

$$
\forall \hat{t} \in \bar{H}_{0}^{\prime}, \quad \bar{b}(\tilde{t}, \hat{t})=\langle u, \hat{t}\rangle_{\bar{H}_{0}, \bar{H}_{0}^{\prime}} .
$$

Setting $\tilde{t}=\overline{\mathscr{L}}(u)$, we obtain a continuous linear mapping from $\bar{H}_{0}$ onto $\bar{H}_{0}^{\prime}$ (take $\tilde{t} \in \bar{H}_{0}^{\prime}$ arbitrary, then $\hat{t} \mapsto \bar{b}(\tilde{t}, \hat{t})$ defines a continuous linear form $u \in \bar{H}_{0}^{\prime \prime}=\bar{H}_{0}$ and we have $\tilde{t}=\overline{\mathscr{L}}(u))$. The space $H_{0}=H^{1 / 2} / \mathscr{C}$ is identified to a closed subspace (with codimension 1) of $\bar{H}_{0}$. Thanks to theorem 36, the restriction of ${ }_{635} \overline{\mathscr{L}}$ to $W^{1, \infty}(-1,1) / \mathscr{C}$ is given by:

$$
\overline{\mathscr{L}}(u)=\frac{s(x) u^{\prime}(x)}{1+s^{2}(x)}-\frac{e^{\tau(x)}}{(1-x) \sqrt{1+s^{2}(x)}} \frac{1}{\pi} \oint_{-1}^{1} \frac{u^{\prime}\left(x^{\prime}\right)\left(1-x^{\prime}\right) e^{-\tau\left(x^{\prime}\right)}}{\sqrt{1+s^{2}\left(x^{\prime}\right)}} \cdot \frac{\mathrm{d} x^{\prime}}{x^{\prime}-x},
$$


where:

$$
\tau(x)=\frac{1}{\pi} \oint_{-1}^{1} \arctan \left(-1 / s\left[s\left(x^{\prime}\right)\right) \frac{\mathrm{d} x^{\prime}}{x^{\prime}-x}\right.
$$

Recalling:

$$
t_{0}(x)=\frac{e^{\tau(x)}}{\pi(1-x) \sqrt{1+s^{2}(x)}},
$$

any $t \in H^{-1 / 2}(-1,1)$ can be decomposed into:

$$
t=\overline{\mathscr{L}}(u)+t_{0}\left\langle t, \chi_{]-1,1\rceil}\right\rangle_{H^{-1 / 2}, H^{1 / 2}},
$$

for some $u \in \bar{H}_{0}$.

640 Remark 1. The step function $\chi_{] 0,1[}+C$ is in $\bar{H}_{0}$ and the question of what $\tau \stackrel{\text { def }}{=} \overline{\mathscr{L}}\left(\chi_{] 0,1[}+C\right) \in \bar{H}_{0}^{\prime}$ is arises. It satisfies:

$$
\left.-\frac{1}{\pi} \tau * \operatorname{vp} \frac{1}{x}+s \tau=\delta, \quad \text { in }\right]-1,1[,
$$

where vp $1 / x$ is the distributional derivative of $\log |x|$ and $\delta$ the Dirac measure. Since the Hilbert transform maps $L^{p}(-1,1)$ into $L^{p}(-1,1)$ for all $\left.p \in\right] 1, \infty[$, it is readily checked that:

$$
\forall p \in] 1, \infty\left[, \quad \tau \notin L^{p}(-1,1) .\right.
$$

I do not know any explicit expression for $\tau$.

Remark 2. In problem $\mathrm{II}^{\prime}$, the function $\bar{\psi}$ describing the geometry of the obstacle is allowed to be not only in $H^{1 / 2}(-1,1) / \mathscr{C}$ but in $\bar{H}_{0}$. In particular, $\bar{\psi}$ may have a jump at 0 , which is not allowed in problem $\mathrm{I}^{\prime}$. Hence, the new functional framework displayed in section 4.1 is appropriate to deal with 650 discontinuities not only in the friction coefficient, but also in the shape of the obstacle.

The bilinear form:

$$
\bar{a}\left(u_{1}, u_{2}\right) \stackrel{\text { def }}{=}\left\langle u_{1}, \overline{\mathscr{L}}\left(u_{2}\right)\right\rangle=\bar{b}\left(\overline{\mathscr{L}}\left(u_{1}\right), \overline{\mathscr{L}}\left(u_{2}\right)\right),
$$

is continuous and coercive on $\bar{H}_{0}$ (we recall that $\bar{H}_{0}$ is the quotient space $\bar{H} / \mathscr{C}$ where $\mathscr{C}$ is the line of all the constant functions on $]-1,1[$ ). It is recalled that an 655 element $\hat{u} \in \bar{H}_{0}$ will be said nonpositive (notation $\hat{u} \leq 0$ ) if one (and therefore all) element of its equivalence class is (essentially) bounded by above. We define:

$$
\bar{K}_{0} \stackrel{\text { def }}{=}\left\{\hat{u} \in \bar{H}_{0} \mid \hat{u}-\bar{\psi} \leq 0\right\},
$$


which is a nonempty closed convex subset of $\bar{H}_{0}$ and:

$$
\begin{aligned}
\varphi(\hat{u}) \stackrel{\text { def }}{=} \mid \begin{array}{ll}
\inf _{C \mid \hat{u}-\bar{\psi}+C \leq 0}\left\langle-P t_{0}, \hat{u}-\bar{\psi}+C\right\rangle_{\bar{H}^{\prime}, \bar{H}}, & \text { if } \hat{u} \in \bar{K}_{0}, \\
+\infty, & \text { otherwise, }
\end{array} \\
=\mid \begin{array}{ll}
\left\langle-P t_{0}, \bar{u}_{\mathrm{n}}-\bar{\psi}-\underset{]-1,1[}{\operatorname{ess} \sup (\hat{u}-\bar{\psi})\rangle_{\bar{H}^{\prime}, \bar{H}},}\right. & \text { if } \hat{u} \in \bar{K}_{0}, \\
+\infty, & \text { otherwise, }
\end{array}
\end{aligned}
$$

where $P \geq 0$ is assumed and where $t_{0}(x)$ is the function defined by formula (19). Note that the functional $\varphi$ is well-defined over the space $\bar{H}_{0}$. Lemma 4 is readily seen to extend to this new definition of $\varphi$, so that $\varphi$ is proper, lower 660 semicontinuous on $\bar{H}_{0}$ and convex. Making use of proposition 5 (which can be readily seen to extend to the case where $\bar{\psi} \in \bar{H}_{0}$ ), we obtain the dual formulation of problem II':

Problem II. Let $P \in \mathbb{R}$ be positive, and $\bar{\psi} \in \bar{H}_{0} \supset H^{1 / 2}(-1,1) / \mathscr{C}$. Find $\bar{u}_{\mathrm{n}} \in \bar{K}_{0}$ such that:

$$
\forall \hat{u} \in \bar{K}_{0}, \quad \bar{a}\left(\hat{u}-\bar{u}_{\mathrm{n}}, \bar{u}_{\mathrm{n}}\right)+\varphi(\hat{u})-\varphi\left(\bar{u}_{\mathrm{n}}\right) \geq 0 .
$$

665 Proposition 15. Assume that $s_{+}>s_{-}$, then problem II has a unique solution. The solution $\bar{u}_{\mathrm{n}} \in \bar{H}_{0}$ of problem II belongs actually to $H^{1 / 2}(-1,1) / \mathscr{C}$. The solutions $t_{n}$ and $\bar{u}_{n}$ of problems $I I^{\prime}$ and II are linked by the relationship:

$$
\tilde{t}_{\mathrm{n}}=\overline{\mathscr{L}}\left(\bar{u}_{\mathrm{n}}\right) .
$$

Proof. The bilinear form $\bar{a}$ is continuous and coercive on $\bar{H}_{0}$. The extension of the Lions-Stampacchia theorem that is provided in appendix A (theorem 25)

670 yields a unique solution of problem II. By proposition 5 (which can be readily seen to extend to the case where $\bar{\psi} \in \bar{H}_{0}$ ), we obtain easily:

$$
\tilde{t}_{\mathrm{n}}=\overline{\mathscr{L}}\left(\bar{u}_{\mathrm{n}}\right) .
$$

Since $\bar{u}_{\mathrm{n}} \in \bar{H}_{0}=\left(H^{1 / 2}+\mathbb{R} \chi_{] 0,1[}\right) / \mathscr{C}$, we have $\bar{u}_{\mathrm{n}}^{\prime}=\tilde{u}_{\mathrm{n}}^{\prime}+C \delta$, for some $\tilde{u}_{\mathrm{n}} \in H^{1 / 2} / \mathscr{C}$ and $C \in \mathbb{R}$. Therefore:

$$
\left.-\frac{1}{\pi} \tilde{t}_{\mathrm{n}} * \operatorname{vp} \frac{1}{x}-\tilde{u}_{\mathrm{n}}^{\prime}=C \delta-s \tilde{t}_{\mathrm{n}}, \quad \text { in }\right]-1,1[,
$$

where $\operatorname{vp} 1 / x$ is the distributional derivative of $\log |x|$ and $\delta$ the Dirac measure. 675 But, $\tilde{t}_{\mathrm{n}}$ being in $H^{-1 / 2}(-1,1)$ and $\tilde{u}_{\mathrm{n}}$ being in $H^{1 / 2}(-1,1) / \mathscr{C}$, the left member of this equality belongs to $H_{00}^{1 / 2^{\prime}}(-1,1)$. Since $t_{\mathrm{n}}$ is a nonpositive distribution 
and is therefore a measure on ] $-1,1$ [ which has finite total mass, $\tilde{t}_{\mathrm{n}}$ is a Radon measure which belongs to $H^{-1 / 2}(-1,1)$. Hence the right member of the equality is a Radon measure. Thanks to proposition 35, this measure has no atom. Since $\tilde{t}_{\mathrm{n}}$ and therefore $s \tilde{t}_{\mathrm{n}}$ have no atoms, we necessarily have $C=0$ and the expected conclusion follows.

Summing up, we have proved the following theorem.

Theorem 16. Let $P \in \mathbb{R}$ be positive, $s:]-1,1[\rightarrow \mathbb{R}$ the function defined by $s=s_{-} \chi_{]-1,0[}+s_{+} \chi_{] 0,1[}$ with $s_{-}<s_{+}$and $\bar{\psi} \in H^{1 / 2}(-1,1)+\mathbb{R} \chi_{] 0,1[}$. Then,

there exists a unique $\left(\bar{u}_{\mathrm{n}}, t_{\mathrm{n}}\right) \in H^{1 / 2}(-1,1) \times \bar{H}^{\prime}$ such that:

$$
\begin{aligned}
& \left.-\frac{1}{\pi} t_{\mathrm{n}} * \mathrm{pv} 1 / x+s t_{\mathrm{n}}=\bar{u}_{\mathrm{n}}^{\prime}, \quad \text { in }\right]-1,1[, \\
& \bar{u}_{\mathrm{n}}-\bar{\psi} \leq 0, \quad t_{\mathrm{n}} \leq 0, \quad\left(\bar{u}_{\mathrm{n}}-\bar{\psi}\right) t_{\mathrm{n}}=0 \\
& \left\langle t_{\mathrm{n}}, \chi_{]-1,1[}\right\rangle_{H^{-1 / 2}, H^{1 / 2}}=-P .
\end{aligned}
$$

Finally, if $\bar{\psi}$ is replaced by $\bar{\psi}+C(C \in \mathbb{R})$, then the corresponding solution is $\left(\bar{u}_{\mathrm{n}}+C, t_{\mathrm{n}}\right)$.

The solution of problem II' $^{\prime}$ is explicit in the case where the moving obstacle is a rigid flat punch $(\bar{\psi} \equiv 0)$. Based on theorem 36 , the explicit solution in that case, is given by

$$
t_{\mathrm{n}}(x)=-P t_{0}(x),
$$

where $t_{0}(x)$ is the function defined by formula (19). This distribution of surface traction under the punch exhibits a singularity at the place where a jump in the friction coefficient occurs. More precisely, the function $t_{0}(x)$ behaves in the neighbourhood of $x=0$ as:

$$
\begin{aligned}
t_{0}(x) & \sim \frac{1}{\pi|x|^{|\beta|} \sqrt{1+s_{-}^{2}}}, \quad \text { as } x \rightarrow 0-, \\
t_{0}(x) & \sim \frac{1}{\pi|x|^{|\beta|} \sqrt{1+s_{+}^{2}}}, \quad \text { as } x \rightarrow 0+,
\end{aligned}
$$

695 where

$$
\beta \stackrel{\text { def }}{=} \frac{1}{\pi} \arctan \left(-1 / s_{-}\right)-\frac{1}{\pi} \underset{] 0, \pi[}{\arctan }\left(-1 / s_{+}\right),
$$

is in ]-1,0[ because of the condition $s_{+}>s_{-}$. This asymptotic behaviour is actually universal with respect to the shape of the obstacle, as seen in the next theorem.

Theorem 17. Assume that $\bar{\psi}^{\prime}$ is piecewise Lipschitz-continuous. In the case $700 s_{+}>s_{-}$, if the unique solution of problem II is such that the point $x=0$ (where 
is the jump in the friction coefficient) is an interior point of the contact set (the support of $\left.t_{\mathrm{n}}\right)$, then:

$$
t_{\mathrm{n}}=-\frac{\theta}{|x|^{|\beta|} \sqrt{1+s^{2}(x)}}
$$

where

$$
\beta \stackrel{\text { def }}{=} \frac{1}{\pi} \underset{] 0, \pi[}{\arctan }\left(-1 / s_{-}\right)-\frac{1}{\pi} \underset{] 0, \pi[}{\arctan }\left(-1 / s_{+}\right),
$$

is in $]-1,0$ [ because of the condition $s_{+}>s_{-}$, and $\theta$ is a nonnegative measure on $705]-1,1[$ whose restriction to a neighbourhood of $x=0$ is a continuous continuous function.

Proof. Set:

$$
\bar{\phi}(x)=\bar{\psi}^{\prime}(0-) x \chi_{]-1,0[}(x)+\bar{\psi}^{\prime}(0+) x \chi_{] 0,1[}(x),
$$

where $\chi_{S}$ is the indicator function of the set $S$. We have the decomposition:

$$
t_{\mathrm{n}}=\overline{\mathscr{L}}(\bar{\phi})+\overline{\mathscr{L}}(\bar{\psi}-\bar{\phi})+\overline{\mathscr{L}}\left(\bar{u}_{\mathrm{n}}-\bar{\psi}\right)-P t_{0} .
$$

Since there exists $\epsilon>0$ such that $] c-\epsilon, c+\epsilon$ [ does not meet the support of $\bar{u}_{\mathrm{n}}-\bar{\psi}$, the restriction of $\overline{\mathscr{L}}\left(\bar{u}_{\mathrm{n}}-\bar{\psi}\right)$ to $] c-\epsilon, c+\epsilon\left[\right.$ is the product of $t_{0}$ with some $C^{\infty}$ function. Besides, the function $\bar{\psi}^{\prime}-\bar{\phi}^{\prime}$ is Lipschitz-continuous in a neighbourhood of 0 which entails that the restriction of $\mathscr{L}(\bar{\psi}-\bar{\phi})$ to a neighbourhood of 0 , is the product of $t_{0}$ with a continous function, thanks to formula (18) and proposition 7 of [5]. Therefore, the claim in the theorem holds true for the last three terms in the above expression of $t_{\mathrm{n}}(x)$. It remains only to prove that it also holds true for $\overline{\mathscr{L}}(\bar{\phi})$. But, this is a straightforward consequence of formula (18) and proposition 7 of [5].

\subsection{Analysis of the case $s_{+}=s_{-}$}

In this section, we analyse the case where $s$ is a constant function as in section 3 , but we now admit $\bar{\psi} \in \bar{H}_{0} \supset H_{0}=H^{1 / 2}(-1,1) / \mathscr{C}$. The function $\bar{\psi}$ may therefore have a jump at $x=0$, which was precluded in the case $\bar{\psi} \in H^{1 / 2}(-1,1) / \mathscr{C}$ analysed in section 3 . The necessary adaptation is going to be described.

First, note that the definition (12) of $K_{0}$ still makes sense in the case $\bar{\psi} \in \bar{H}_{0}$ and $K_{0}$ is still a closed convex subset on $H_{0}$. To check that it is nonempty, just set $\bar{\psi}=\tilde{\psi}+C \chi_{] 0,1[}$ with $\tilde{\psi} \in H_{0}$ and note that $\tilde{\psi} \in K_{0}$. Based on this simple observation, the formulation of the problem governing the normal surface displacement remains unchanged.

Problem III. Let $s, P \in \mathbb{R}, P$ be positive, and $\bar{\psi} \in \bar{H}_{0}$. Find $\bar{u}_{\mathrm{n}} \in K_{0}$ such that:

$$
\forall \hat{u} \in K_{0}, \quad a\left(\hat{u}-\bar{u}_{\mathrm{n}}, \bar{u}_{\mathrm{n}}\right)+\varphi(\hat{u})-\varphi\left(\bar{u}_{\mathrm{n}}\right) \geq 0,
$$

where $\varphi$ is the functional defined by formula (13). 
Problem III has a unique solution, thanks to theorem 25 in appendix A. Let us turn now to the dual problem. Note that $K_{0}^{\prime}$ contains only Radon measures with no atom so that actually $\bar{K}_{0}^{\prime}=K_{0}^{\prime}$, and the problem governing the normal surface traction remains unchanged.

Problem III'. Let $\bar{\psi} \in \bar{H}_{0} \supset H^{1 / 2}(-1,1) / \mathscr{C}$. Find $\tilde{t}_{\mathrm{n}} \in \bar{K}_{0}^{\prime}$ such that:

$$
\forall \hat{t} \in \bar{K}_{0}^{\prime}, \quad b\left(\tilde{t}_{\mathrm{n}}, \hat{t}-\tilde{t}_{\mathrm{n}}\right) \geq\left\langle\bar{\psi}, \hat{t}-\tilde{t}_{\mathrm{n}}\right\rangle_{\bar{H}_{0}, \bar{H}_{0}^{\prime}} .
$$

In this case, $b$ is coercive on $H_{0}^{\prime}$, but it is not coercive on $\bar{H}_{0}^{\prime}$, and $\bar{\psi} \in \bar{H}_{0}$, but $\bar{\psi} \notin H_{0}$ in general. Problem III' can therefore not be solved by use of the

740 Lions-Stampacchia theorem. However, Problem III' has a unique solution thanks to theorem 25 and the following lemma.

Lemma 18. The functional defined on $H_{0}^{\prime}$ by:

$$
\varphi^{*}(-\hat{t}) \stackrel{\text { def }}{=} \mid \begin{array}{ll}
\langle-\hat{t}, \bar{\psi}\rangle_{\bar{H}_{0}^{\prime}, \bar{H}_{0}}, & \text { if } \hat{t} \in \bar{K}_{0}^{\prime}, \\
+\infty, & \text { otherwise },
\end{array}
$$

is proper, convex and lower semicontinuous on $H_{0}^{\prime}$.

Proof. First, note that the functional is obviously proper and convex. Now, pick ${ }_{745} t_{\infty} \in \bar{K}_{0}^{\prime}$ and a sequence $\left(t_{n}\right)$ in $\bar{K}_{0}^{\prime}$ that converges strongly in $H_{0}^{\prime}$ towards $t_{\infty}$. The sequence $\left(t_{n}\right)$ must converge weakly-* in the space of measures towards $t_{\infty}$. Hence, the sequence $\left(\int_{0}^{1} t_{n}\right)$ converges towards $\left(\int_{0}^{1} t_{\infty}\right)$. This entails that the sequence $\left(t_{n}\right)$ converges weakly in $\bar{H}_{0}^{\prime}$ towards $t_{\infty}$, and therefore $\lim \varphi^{*}\left(t_{n}\right)=$ $\varphi^{*}\left(t_{\infty}\right)$. This is enough to get the lower semicontinuity of $\varphi^{*}$ on $H_{0}^{\prime}$.

The solutions $\bar{u}_{\mathrm{n}}$ and $\tilde{t}_{\mathrm{n}}$ of problems III and III' are connected by:

$$
\tilde{t}_{\mathrm{n}}=\mathscr{L}\left(\bar{u}_{\mathrm{n}}\right),
$$

thanks to proposition 5 (which can be readily seen to extend to the case where $\left.\bar{\psi} \in \bar{H}_{0}\right)$.

Summing up, theorem 8 holds true not only for $\bar{\psi} \in H^{1 / 2}(-1,1)$, but also for $\bar{\psi} \in H^{1 / 2}(-1,1)+\mathbb{R} \chi_{] 0,1[}$.

\subsection{Analysis of the case $s_{+}<s_{-}$}

In that case, the bilinear form $\bar{b}$ is not coercive in $\bar{H}_{0}^{\prime}$.

To get insight into this case, let us examine the situation of the particular case of the rigid flat punch, that is, $\bar{\psi} \equiv 0$. Reproducing the same reasoning as that in the proof of proposition 2 of [5], it can be proved that any solution of the 
steady sliding frictional contact problem such that $t_{\mathrm{n}} \in \cup_{p>1} L^{p}(-1,1)$ achieves active contact everywhere below the punch: $\bar{u}_{\mathrm{n}} \equiv 0$. Therefore, $t_{\mathrm{n}}$ must solve the following problem.

$$
\begin{aligned}
& \text { - } \left.\frac{1}{\pi} \oint_{-1}^{1} \frac{t_{\mathrm{n}}\left(x^{\prime}\right)}{x^{\prime}-x} \mathrm{~d} x^{\prime}+s(x) t_{\mathrm{n}}(x)=0, \quad \text { for a.a. } x \in\right]-1,1[, \\
& \text { - } \int_{-1}^{1} t_{\mathrm{n}}\left(x^{\prime}\right) \mathrm{d} x^{\prime}=-P .
\end{aligned}
$$

Thanks to theorem 36 in appendix $\mathrm{C}$, all the solutions in $\cup_{p>1} L^{p}(-1,1)$ of this singular integral equation are:

$$
\begin{array}{r}
t_{\mathrm{n}}(x)=-\frac{P e^{\tau(x)}}{(1-x) \sqrt{s^{2}(x)+1}}\left(\int_{-1}^{1} \frac{e^{\tau\left(x^{\prime}\right)} \mathrm{d} x^{\prime}}{\left(1-x^{\prime}\right) \sqrt{1+s^{2}\left(x^{\prime}\right)}}\right)^{-1} \\
+\frac{C e^{\tau(x)}}{\pi x(1-x) \sqrt{s^{2}(x)+1}},
\end{array}
$$

where $C \in \mathbb{R}$ is an arbitrary constant. Note that the identity proved in the step 1 of the proof of theorem 14 of [5] (with the choices $\lambda=-1$ and $x=1$ ), gives:

$$
\int_{-1}^{1} \frac{e^{\tau\left(x^{\prime}\right)} \mathrm{d} x^{\prime}}{\left(1-x^{\prime}\right) \sqrt{1+s^{2}\left(x^{\prime}\right)}}=\pi .
$$

The fact that the above linear problem admits infinitely many solutions is another proof of the fact that the bilinear form $\bar{b}$ is not coercive in $\bar{H}_{0}^{\prime}$. An interesting fact arises: although the linear problem has infinitely many solutions, there is only exactly one of these solutions which is a nonpositive function: that corresponding to the choice $C=0$. Therefore, the steady sliding contact problem has one and only one solution in $\cup_{p>1} L^{p}(-1,1)$. It reads as:

$$
t_{\mathrm{n}}(x)=-\frac{P e^{\tau(x)}}{\pi(1-x) \sqrt{s^{2}(x)+1}} .
$$

It is interesting to note that the asymptotic behaviour of that solution in the neighbourhood of $x=0$ is now given by:

$$
\begin{aligned}
t_{\mathrm{n}}(x) \sim \frac{P|x|^{\beta}}{\pi \sqrt{1+s_{-}^{2}}}, & \text { as } x \rightarrow 0-, \\
t_{\mathrm{n}}(x) \sim \frac{P|x|^{\beta}}{\pi \sqrt{1+s_{+}^{2}}}, \quad \text { as } x \rightarrow 0+, &
\end{aligned}
$$

where

$$
\beta \stackrel{\text { def }}{=} \frac{1}{\pi} \underset{] 0, \pi[}{\arctan }\left(-1 / s_{-}\right)-\frac{1}{\pi} \underset{] 0, \pi[}{\arctan }\left(-1 / s_{+}\right),
$$


is in ] $0,1\left[\right.$ because of the condition $s_{+}<s_{-}$. Therefore, $t_{n}(x)$ is now locally bounded around $x=0$ (see figure 4 ).

We have therefore discovered a fact of engineering importance. In the situation, where a rigid punch with two different coatings (producing two different friction coefficients with a jump at some point) is steadily sliding along the boundary of some linearly elastic body, there is a singularity of the surface traction at the jump in the case where the largest friction coefficient is front, 775 whereas the surface traction is locally bounded at the jump when the largest friction coefficient is rear.

So, in the case $s_{+}<s_{-}$, the bilinear form $\bar{b}$ is coercive neither in $\bar{H}^{\prime}$ nor in $\bar{H}_{0}^{\prime}$. A glance at theorem 36 in appendix $\mathrm{C}$ shows that, in the case $s_{+}<s_{-}$, the functions

$$
C_{0} t_{0}(x)+C_{1} t_{1}(x)=\frac{\left(C_{0} x+C_{1}\right) e^{\tau(x)}}{\pi x(1-x) \sqrt{s^{2}(x)+1}},
$$

where:

$$
\tau(x)=\frac{1}{\pi} \oint_{-1}^{1} \underset{] 0, \pi[}{\arctan }\left(-1 / s\left(x^{\prime}\right)\right) \frac{\mathrm{d} x^{\prime}}{x^{\prime}-x},
$$

and $C_{0}, C_{1} \in \mathbb{R}$, are all in $L^{p}(-1,1)$ for some $p>1$, and satisfy:

$$
\forall C_{0}, C_{1} \in \mathbb{R}, \quad \forall \hat{t} \in \bar{H}_{0}^{\prime}, \quad \bar{b}\left(C_{0} t_{0}+C_{1} t_{1}, \hat{t}\right)=0 .
$$

In addition, $t_{0}$ is positive, the restriction of $t_{1}$ to $]-1,0[$ is negative and the restriction of $t_{1}$ to $] 0,1[$ is positive. Furthermore, we have:

$$
\int_{-1}^{1} t_{0}(x) \mathrm{d} x=1, \quad \int_{-1}^{1} t_{1}(x) \mathrm{d} x=0
$$

Formula (17) shows that $\bar{b}$ is not coercive in $\bar{H}_{0}^{\prime}$. However, it is coercive in 785 the space:

$$
\bar{H}_{00}^{\prime} \stackrel{\text { def }}{=}\left\{t \in \bar{H}^{\prime} \mid \int_{-1}^{0} \tilde{t}=\int_{0}^{1} \tilde{t}=0\right\},
$$

which is clearly a subspace of codimension 2 in $\bar{H}^{\prime}$ and a subspace of codimension 1 in $\bar{H}_{0}^{\prime}$. The restriction of $\bar{b}$ to $\bar{H}_{00}^{\prime}$ is continuous and coercive. The dual space of $\bar{H}_{00}^{\prime}$ is $\bar{H}_{00}=\bar{H}_{0} /\left(\mathbb{R} \chi_{] 0,1[}\right)$ which can be identified to $H_{0}=H^{1 / 2}(-1,1) / \mathscr{C}$. Therefore, for arbitrary $u \in \bar{H}_{00} \simeq H_{0}$, there exists a unique $t \in \bar{H}_{00}^{\prime}$ such that:

$$
\forall \hat{t} \in \bar{H}_{00}^{\prime}, \quad \bar{b}(t, \hat{t})=\langle u, \hat{t}\rangle .
$$

790 Setting $t=\overline{\mathscr{L}}^{\prime}(u)$, we obtain a continuous linear mapping from $\bar{H}_{00}$ onto $\bar{H}_{00}^{\prime}$. The distribution $t$ satisfies:

$$
\left.-\frac{1}{\pi} t * \operatorname{pv} 1 / x+s t=u^{\prime}+C \delta, \quad \text { in }\right]-1,1[,
$$


for some $C \in \mathbb{R}$. In the sequel, it will be desirable to ensure that $C=0$. This fact is equivalent to:

$$
\forall \hat{t} \in \bar{H}_{0}^{\prime}, \quad \bar{b}(t, \hat{t})=\langle u, \hat{t}\rangle,
$$

which is itself equivalent to $\bar{b}\left(t, t_{1}\right)=\left\langle u, t_{1}\right\rangle$, due to the fact that $\bar{H}_{0}^{\prime}=\bar{H}_{00}^{\prime}+\mathbb{R} t_{1}$.

Proposition 19. The unique solution $t=\overline{\mathscr{L}}^{\prime}(u)$ in $\bar{H}_{00}^{\prime}$ of the variational equality:

$$
\forall \hat{t} \in \bar{H}_{00}^{\prime}, \quad \bar{b}(t, \hat{t})=\langle u, \hat{t}\rangle,
$$

actually satisfies:

$$
\forall \hat{t} \in \bar{H}_{0}^{\prime}, \quad \bar{b}(t, \hat{t})=\langle u, \hat{t}\rangle .
$$

Furthermore, for $u \in W^{1, \infty}(-1,1) / \mathscr{C} \subset H^{1 / 2}(-1,1) / \mathscr{C}=H_{0}, \overline{\mathscr{L}}^{\prime}(u) \in \bar{H}_{00}^{\prime}$ is given by:

$$
\begin{aligned}
\overline{\mathscr{L}}^{\prime}(u)= & \frac{s(x) u^{\prime}(x)}{1+s^{2}(x)} \\
& -\frac{e^{\tau(x)}}{(1-x) \sqrt{1+s^{2}(x)}} \frac{1}{\pi} \oint_{-1}^{1} \frac{u^{\prime}\left(x^{\prime}\right)\left(1-x^{\prime}\right) e^{-\tau\left(x^{\prime}\right)}}{\sqrt{1+s^{2}\left(x^{\prime}\right)}} \cdot \frac{\mathrm{d} x^{\prime}}{x^{\prime}-x} \\
& +C_{1}(u) t_{1}(x),
\end{aligned}
$$

where:

$$
t_{1}(x)=\frac{e^{\tau(x)}}{\pi x(1-x) \sqrt{s^{2}(x)+1}}, \quad \tau(x)=\frac{1}{\pi} \oint_{-1}^{1} \underset{] 0, \pi[}{\arctan }\left(-1 / s\left(x^{\prime}\right)\right) \frac{\mathrm{d} x^{\prime}}{x^{\prime}-x},
$$

and $C_{1}(u)$ is a real constant which is uniquely determined by the condition $\overline{\mathscr{L}}^{\prime}(u) \in \bar{H}_{00}^{\prime}$, that is, $\int_{0}^{1} \overline{\mathscr{L}}^{\prime}(u)=0$.

Proof. Taking $u$ in $W^{1, \infty}(-1,1)$, the general solution $\tau \in \cup_{q>1} L^{q}(-1,1)$ of the singular integral equation:

$$
\left.\frac{1}{\pi} \oint_{-1}^{1} \frac{\tau\left(x^{\prime}\right)}{x^{\prime}-x} \mathrm{~d} x^{\prime}+s(x) \tau(x)=u^{\prime}(x), \quad \text { for a.a. } x \in\right]-1,1[,
$$

is given by:

$$
\begin{aligned}
\tau= & \frac{s(x) u^{\prime}(x)}{1+s^{2}(x)}-\frac{e^{\tau(x)}}{(1-x) \sqrt{1+s^{2}(x)}} \frac{1}{\pi} \oint_{-1}^{1} \frac{u^{\prime}\left(x^{\prime}\right)\left(1-x^{\prime}\right) e^{-\tau\left(x^{\prime}\right)}}{\sqrt{1+s^{2}\left(x^{\prime}\right)}} \cdot \frac{\mathrm{d} x^{\prime}}{x^{\prime}-x} \\
& +\frac{\left[x C_{0}+C_{1}\right] e^{\tau(x)}}{\pi x(1-x) \sqrt{s^{2}(x)+1}}, \\
= & \frac{s(x) u^{\prime}(x)}{1+s^{2}(x)}-\frac{e^{\tau(x)}}{x(1-x) \sqrt{1+s^{2}(x)}} \frac{1}{\pi} \oint_{-1}^{1} \frac{u^{\prime}\left(x^{\prime}\right) x^{\prime}\left(1-x^{\prime}\right) e^{-\tau\left(x^{\prime}\right)}}{\sqrt{1+s^{2}\left(x^{\prime}\right)}} \cdot \frac{\mathrm{d} x^{\prime}}{x^{\prime}-x} \\
& +\frac{\left[x C_{0}+C_{1}^{\prime}\right] e^{\tau(x)}}{\pi x(1-x) \sqrt{s^{2}(x)+1}},
\end{aligned}
$$


thanks to theorem 36 of appendix C. Invoking theorem 11 (i) of [5], it can be readily checked that:

$$
\begin{aligned}
& \int_{-1}^{1}\left\{\frac{s(x) u^{\prime}(x)}{1+s^{2}(x)}\right. \\
& \left.\quad-\frac{e^{\tau(x)}}{(1-x) \sqrt{1+s^{2}(x)}} \frac{1}{\pi} \oint_{-1}^{1} \frac{u^{\prime}\left(x^{\prime}\right)\left(1-x^{\prime}\right) e^{-\tau\left(x^{\prime}\right)}}{\sqrt{1+s^{2}\left(x^{\prime}\right)}} \cdot \frac{\mathrm{d} x^{\prime}}{x^{\prime}-x}\right\} \mathrm{d} x=0, \\
& \int_{-1}^{1}\left\{\frac{s(x) u^{\prime}(x)}{1+s^{2}(x)}\right. \\
& \left.\quad-\frac{e^{\tau(x)}}{x(1-x) \sqrt{1+s^{2}(x)}} \frac{1}{\pi} \oint_{-1}^{1} \frac{u^{\prime}\left(x^{\prime}\right) x^{\prime}\left(1-x^{\prime}\right) e^{-\tau\left(x^{\prime}\right)}}{\sqrt{1+s^{2}\left(x^{\prime}\right)}} \cdot \frac{\mathrm{d} x^{\prime}}{x^{\prime}-x}\right\} \mathrm{d} x=0 .
\end{aligned}
$$

Therefore:

$$
\begin{aligned}
\bar{t} \stackrel{\text { def }}{=} & \tau-t_{0} \int_{-1}^{1} \tau \\
= & \frac{s(x) u^{\prime}(x)}{1+s^{2}(x)}-\frac{e^{\tau(x)}}{(1-x) \sqrt{1+s^{2}(x)}} \frac{1}{\pi} \oint_{-1}^{1} \frac{u^{\prime}\left(x^{\prime}\right)\left(1-x^{\prime}\right) e^{-\tau\left(x^{\prime}\right)}}{\sqrt{1+s^{2}\left(x^{\prime}\right)}} \cdot \frac{\mathrm{d} x^{\prime}}{x^{\prime}-x}+C_{1} t_{1}, \\
= & \frac{s(x) u^{\prime}(x)}{1+s^{2}(x)}-\frac{e^{\tau(x)}}{x(1-x) \sqrt{1+s^{2}(x)}} \frac{1}{\pi} \oint_{-1}^{1} \frac{u^{\prime}\left(x^{\prime}\right) x^{\prime}\left(1-x^{\prime}\right) e^{-\tau\left(x^{\prime}\right)}}{\sqrt{1+s^{2}\left(x^{\prime}\right)}} \cdot \frac{\mathrm{d} x^{\prime}}{x^{\prime}-x} \\
& +C_{1}^{\prime} t_{1}
\end{aligned}
$$

belongs to $\bar{H}_{0}^{\prime}$. There is only one value of the constant $C_{1}$ such that it actually belongs to $\bar{H}_{00}^{\prime}$. Making that choice, we adopt the notation $\tilde{t} \in \bar{H}_{00}^{\prime}$ for $\bar{t}$. It 805 satisfies:

$$
-\frac{1}{\pi} \int_{-1}^{1} \tilde{t}\left(x^{\prime}\right) \log \left|x^{\prime}-x\right| \mathrm{d} x^{\prime}+\int_{-1}^{x} s\left(x^{\prime}\right) \tilde{t}\left(x^{\prime}\right) \mathrm{d} x^{\prime}=u(x)+C,
$$

for all $x \in]-1,1[$ and some constant $C \in \mathbb{R}$. Therefore:

$$
\forall \hat{t} \in \bar{H}_{0}^{\prime}, \quad \bar{b}(\bar{t}, \hat{t})=\langle u, \hat{t}\rangle,
$$

which is the expected conclusion $\tilde{t}=\overline{\mathscr{L}}^{\prime}(u)$.

Next, take $u \in H_{0}=H^{1 / 2}(-1,1) / \mathscr{C}$ arbitrary and let $t=\overline{\mathscr{L}}^{\prime}(u) \in \bar{H}_{00}^{\prime}$ be the unique solution of the variational equality:

$$
\forall \hat{t} \in \bar{H}_{00}^{\prime}, \quad \bar{b}(t, \hat{t})=\langle u, \hat{t}\rangle .
$$

810 We have to prove:

$$
\bar{b}\left(t, t_{1}\right)=\left\langle u, t_{1}\right\rangle .
$$


We define:

$$
l(u)=\bar{b}\left(\overline{\mathscr{L}}^{\prime}(u), t_{1}\right)-\left\langle u, t_{1}\right\rangle,
$$

which is clearly a continuous linear form on $H_{0}=H^{1 / 2}(-1,1) / \mathscr{C}$. Thanks to the first part of the proof, this continuous linear form vanishes identically on $W^{1, \infty}(-1,1) / \mathscr{C}$. Since $W^{1, \infty}(-1,1)$ is dense in $H^{1 / 2}(-1,1)$, the continuous 815 linear form $l(u)$ vanishes identically on $H_{0}=H^{1 / 2}(-1,1) / \mathscr{C}$. This is the expected conclusion.

The set:

$$
\bar{K}_{00}^{\prime} \stackrel{\text { def }}{=}\left\{\hat{t} \in \bar{H}_{00}^{\prime} \mid \exists F \in \mathbb{R}, \quad \hat{t}+F t_{1}-P t_{0} \leq 0\right\},
$$

is clearly a closed convex subset of $\bar{H}_{00}^{\prime}$. In the case where $P \geq 0$, it is nonempty because it contains 0 . Set:

$$
\phi^{*}(\hat{t}) \stackrel{\text { def }}{=} \mid \begin{array}{lr}
\inf _{F \mid \hat{t}+F t_{1} \leq P t_{0}}\left\langle-\bar{\psi}, \hat{t}+F t_{1}\right\rangle_{\bar{H}_{0}, \bar{H}_{0}^{\prime},}, \quad \text { if } \hat{t} \in \bar{K}_{00}^{\prime}, \\
+\infty, & \text { otherwise. }
\end{array}
$$

820 Proposition 20. The functional $\phi^{*}$ is proper, lower semicontinuous on $\bar{H}_{00}^{\prime}$ and convex.

Proof. Recalling that $t_{0}$ is positive on $]-1,1\left[, t_{1}\right.$ is negative on $]-1,0[$ and positive on ]0,1[, we have:

$$
\begin{aligned}
& \hat{t}+F t_{1}-P t_{0} \leq 0, \text { with } \hat{t} \in \bar{H}_{00}^{\prime} \quad \Longrightarrow \\
& \qquad-P\left(\int_{-1}^{0} t_{0}\right) /\left(\int_{0}^{1} t_{1}\right) \leq F \leq P\left(\int_{0}^{1} t_{0}\right) /\left(\int_{0}^{1} t_{1}\right) .
\end{aligned}
$$

Therefore, the infimum in the definition of $\phi^{*}$ is taken over a closed subset of a bounded real interval and is therefore achieved so that this infimum is actually a minimum. Taking $\hat{t}_{1}, \hat{t}_{2}$ arbitrary in $\bar{K}_{00}^{\prime}$, we therefore have:

$$
\begin{aligned}
& \left\langle-\bar{\psi}, \hat{t}_{1}+F_{1} t_{1}\right\rangle_{\bar{H}_{0}, \bar{H}_{0}^{\prime}}=\inf _{F \mid \hat{t}_{1}+F t_{1} \leq P t_{0}}\left\langle-\bar{\psi}, \hat{t}_{1}+F t_{1}\right\rangle_{\bar{H}_{0}, \bar{H}_{0}^{\prime}}, \\
& \left\langle-\bar{\psi}, \hat{t}_{2}+F_{2} t_{1}\right\rangle_{\bar{H}_{0}, \bar{H}_{0}^{\prime}}=\inf _{F \mid \hat{t}_{2}+F t_{1} \leq P t_{0}}\left\langle-\bar{\psi}, \hat{t}_{2}+F t_{1}\right\rangle_{\bar{H}_{0}, \bar{H}_{0}^{\prime}},
\end{aligned}
$$

so that:

$$
\forall \lambda \in[0,1], \quad \lambda \hat{t}_{1}+(1-\lambda) \hat{t}_{2}+\left[\lambda F_{1}+(1-\lambda) F_{2}\right] t_{1} \leq P t_{0},
$$

and therefore:

$$
\forall \lambda \in[0,1], \quad \phi^{*}\left(\lambda \hat{t}_{1}+(1-\lambda) \hat{t}_{2}\right) \leq \lambda \phi^{*}\left(\hat{t}_{1}\right)+(1-\lambda) \phi^{*}\left(\hat{t}_{2}\right),
$$


that is, the functional $\phi^{*}$ is convex. We will now prove that it is also lower semicontinuous. Let $\hat{t}_{\infty} \in \bar{K}_{00}^{\prime}$ and a sequence $\left(\hat{t}_{k}\right)_{k \in \mathbb{N}}$ in $\bar{K}_{00}^{\prime}$ converging strongly towards $\hat{t}_{\infty}$. Set:

$$
\left\langle-\bar{\psi}, \hat{t}_{k}+F_{k} t_{1}\right\rangle_{\bar{H}_{0}, \bar{H}_{0}^{\prime}}=\inf _{F \mid \hat{t}_{k}+F t_{1} \leq P t_{0}}\left\langle-\bar{\psi}, \hat{t}_{k}+F t_{1}\right\rangle_{\bar{H}_{0}, \bar{H}_{0}^{\prime}} \cdot
$$

We have:

$$
\liminf _{k \rightarrow+\infty}\left\langle-\bar{\psi}, \hat{t}_{k}+F_{k} t_{1}\right\rangle_{\bar{H}_{0}, \bar{H}_{0}^{\prime}}=\left\langle-\bar{\psi}, \hat{t}_{\infty}+F_{\infty} t_{1}\right\rangle_{\bar{H}_{0}, \bar{H}_{0}^{\prime}},
$$

for some $F_{\infty}$ satisfying:

$$
\hat{t}_{\infty}+F_{\infty} t_{1} \leq P t_{0}
$$

Therefore:

$$
\phi^{*}\left(\hat{t}_{\infty}\right) \leq \liminf _{k \rightarrow+\infty} \phi^{*}\left(\hat{t}_{k}\right),
$$

that is, $\phi^{*}$ is lower semicontinuous.

Problem IV'. Let $\bar{\psi} \in \bar{H}_{0} \supset H^{1 / 2}(-1,1) / \mathscr{C}$. Find $\tilde{t}_{\mathrm{n}} \in \bar{K}_{00}^{\prime}$ such that:

$$
\forall \hat{t} \in \bar{K}_{00}^{\prime}, \quad \bar{b}\left(\tilde{t}_{\mathrm{n}}, \hat{t}-\tilde{t}_{\mathrm{n}}\right)+\phi^{*}(\hat{t})-\phi^{*}\left(\tilde{t}_{\mathrm{n}}\right) \geq 0 .
$$

Problem IV' has a unique solution, thanks to proposition 20 and theorem 25. The following proposition provides a pointwise interpretation of the solution of problem IV'.

${ }_{835}$ Proposition 21. Let $P \in \mathbb{R}$ be positive, $\bar{u}_{\mathrm{n}} \in H_{0}=H^{1 / 2}(-1,1) / \mathbb{R}$ and $\tilde{t}_{\mathrm{n}} \in$ $\bar{H}_{00}^{\prime}$. Then, the two following statements are equivalent.

(i) $\tilde{t}_{\mathrm{n}} \in \bar{K}_{00}^{\prime}, \quad$ and $\quad \forall \hat{t} \in \bar{K}_{00}^{\prime}, \quad\left\langle\bar{u}_{\mathrm{n}}, \hat{t}-\tilde{t}_{\mathrm{n}}\right\rangle_{\bar{H}_{00}, \bar{H}_{00}^{\prime}}+\phi^{*}(\hat{t})-\phi^{*}\left(\tilde{t}_{\mathrm{n}}\right) \geq 0$,

(ii) The function $\bar{u}_{\mathrm{n}}-\bar{\psi}$ is essentially bounded by above and there exists $\tilde{F} \in \mathbb{R}$ such that the three following statements hold true in $]-1,1[$ :

$$
\begin{aligned}
& \bar{u}_{\mathrm{n}}-\frac{\int_{-1}^{1} t_{1} \bar{u}_{\mathrm{n}}}{\int_{0}^{1} t_{1}} \chi_{] 0,1[}-\bar{\psi}-\underset{]-1,1[}{\operatorname{ess} \sup }\left(\bar{u}_{\mathrm{n}}-\frac{\int_{-1}^{1} t_{1} \bar{u}_{\mathrm{n}}}{\int_{0}^{1} t_{1}} \chi_{] 0,1[}-\bar{\psi}\right) \leq 0, \\
& \tilde{t}_{\mathrm{n}}+\tilde{F} t_{1}-P t_{0} \leq 0, \\
& {\left[\bar{u}_{\mathrm{n}}-\frac{\int_{-1}^{1} t_{1} \bar{u}_{\mathrm{n}}}{\int_{0}^{1} t_{1}} \chi_{] 0,1[}-\bar{\psi}-\underset{]-1,1[}{\operatorname{ess} \sup }\left(\bar{u}_{\mathrm{n}}-\frac{\int_{-1}^{1} t_{1} \bar{u}_{\mathrm{n}}}{\int_{0}^{1} t_{1}} \chi_{] 0,1[}-\bar{\psi}\right)\right] \times} \\
& \times\left[\tilde{t}_{\mathrm{n}}+\tilde{F} t_{1}-P t_{0}\right]=0 .
\end{aligned}
$$




\section{Proof.}

(ii) $\Rightarrow$ (i). Pick $\hat{t} \in \bar{K}_{00}^{\prime}$ arbitrarily. Since the infimum is taken over a closed subset of a bounded interval, there exists $\hat{F} \in \mathbb{R}$ such that:

$$
\begin{aligned}
& \left\langle-\bar{\psi}, \hat{t}+\hat{F} t_{1}\right\rangle_{\bar{H}_{0}, \bar{H}_{0}^{\prime}}=\inf _{F \mid \hat{t}+F t_{1} \leq P t_{0}}\left\langle-\bar{\psi}, \hat{t}+F t_{1}\right\rangle_{\bar{H}_{0}, \bar{H}_{0}^{\prime}}, \\
& \hat{t}+\hat{F} t_{1} \leq P t_{0} .
\end{aligned}
$$

Therefore:

$$
\begin{aligned}
{\left[\bar{u}_{\mathrm{n}}-\frac{\int_{-1}^{1} t_{1} \bar{u}_{\mathrm{n}}}{\int_{0}^{1} t_{1}} \chi_{] 0,1[}-\bar{\psi}-\underset{]-1,1[}{\operatorname{ess} \sup }\right.} & \left.\left(\bar{u}_{\mathrm{n}}-\frac{\int_{-1}^{1} t_{1} \bar{u}_{\mathrm{n}}}{\int_{0}^{1} t_{1}} \chi_{] 0,1[}-\bar{\psi}\right)\right] \times \\
\times & {\left[\hat{t}+\hat{F} t_{1}-P t_{0}-\tilde{t}_{\mathrm{n}}-\tilde{F} t_{1}+P t_{0}\right] \geq 0 . }
\end{aligned}
$$

Integrating over $]-1,1[$, we get:

$$
\left\langle\bar{u}_{\mathrm{n}}-\frac{\int_{-1}^{1} t_{1} \bar{u}_{\mathrm{n}}}{\int_{0}^{1} t_{1}} \chi_{] 0,1[}-\bar{\psi}, \hat{t}+\hat{F} t_{1}-\tilde{t}_{\mathrm{n}}-\tilde{F} t_{1}\right\rangle_{\bar{H}_{0}, \bar{H}_{0}^{\prime}} \geq 0,
$$

that is:

$$
\left\langle\bar{u}_{\mathrm{n}}, \hat{t}-\tilde{t}\right\rangle+\phi^{*}(\hat{t}) \geq\left\langle-\bar{\psi}, \tilde{t}+\tilde{F} t_{1}\right\rangle \geq \inf _{F \mid \tilde{t}_{\mathrm{n}}+F t_{1} \leq P t_{0}}\left\langle-\bar{\psi}, \tilde{t}_{\mathrm{n}}+F t_{1}\right\rangle=\phi^{*}\left(\tilde{t}_{\mathrm{n}}\right),
$$

which is nothing but (i).

(i) $\Rightarrow$ (ii). So we assume that (i) is true. There exists $\tilde{F} \in \mathbb{R}$ such that:

$$
\begin{aligned}
& \left\langle-\bar{\psi}, \tilde{t}_{\mathrm{n}}+\tilde{F} t_{1}\right\rangle_{\bar{H}_{0}, \bar{H}_{0}^{\prime}}=\inf _{F \mid \tilde{t}_{\mathrm{n}}+F t_{1} \leq P t_{0}}\left\langle-\bar{\psi}, \tilde{t}_{\mathrm{n}}+F t_{1}\right\rangle_{\bar{H}_{0}, \bar{H}_{0}^{\prime}}, \\
& \tilde{t}_{\mathrm{n}}+\tilde{F} t_{1} \leq P t_{0},
\end{aligned}
$$

and we obtain:

$\forall \hat{t} \in \bar{K}_{00}^{\prime}, \quad \forall \hat{F}$ such that $\hat{t}+\hat{F} t_{1} \leq P t_{0}$,

$$
\left\langle\bar{u}_{\mathrm{n}}, \hat{t}-\tilde{t}_{\mathrm{n}}\right\rangle_{\bar{H}_{0}, \bar{H}_{0}^{\prime}}-\left\langle\bar{\psi}, \hat{t}+\hat{F} t_{1}-\tilde{t}_{\mathrm{n}}-\tilde{F} t_{1}\right\rangle_{\bar{H}_{0}, \bar{H}_{0}^{\prime}} \geq 0 .
$$

Recalling the notation:

$$
\bar{K}_{0}^{\prime}=\left\{\hat{t} \in \bar{H}_{0}^{\prime} \mid \hat{t} \leq P t_{0}\right\}
$$

we actually have:

$$
\forall \hat{t} \in \bar{K}_{0}^{\prime}, \quad\left\langle\bar{u}_{\mathrm{n}}-\frac{\int_{-1}^{1} t_{1} \bar{u}_{\mathrm{n}}}{\int_{0}^{1} t_{1}} \chi_{] 0,1[}-\bar{\psi}, \hat{t}-\tilde{t}-\tilde{F} t_{1}\right\rangle_{\bar{H}_{0}, \bar{H}_{0}^{\prime}} \geq 0 .
$$


Thanks to proposition 5 (which can be readily seen to extend to the situation where $H_{0}$ is replaced by $\bar{H}_{0}$ ), this entails that (ii) holds true.

As previously, problem IV ${ }^{\prime}$ can be given an equivalent formulation in terms of displacement (primal formulation). We will systematically identify $\bar{H}_{00}$ with $H_{0}=H^{1 / 2}(-1,1) / \mathbb{R}$. The bilinear form:

$$
\bar{a}\left(u_{1}, u_{2}\right) \stackrel{\text { def }}{=}\left\langle u_{1}, \overline{\mathscr{L}}^{\prime}\left(u_{2}\right)\right\rangle_{\bar{H}_{00}, \bar{H}_{00}^{\prime}}=\bar{b}\left(\overline{\mathscr{L}}^{\prime}\left(u_{1}\right), \overline{\mathscr{L}}^{\prime}\left(u_{2}\right)\right),
$$

is continuous and coercive on $\bar{H}_{00}$. The conjugate of $\phi^{*}$ by the Legendre-Fenchel 850 transform is defined by:

$$
\phi(\hat{u})=\sup _{\hat{t} \in \bar{H}_{00}^{\prime}}\left\{\langle\hat{t}, \hat{u}\rangle-\phi^{*}(\hat{t})\right\}
$$

Since $\phi^{*}$ is proper, convex and lower semicontinuous on $\bar{H}_{00}^{\prime}$ (proposition 20), the functional $\phi$ is proper, convex and lower semicontinuous on $\bar{H}_{00} \simeq H_{0}$. Hence, theorem 25 yields a unique solution of the following problem.

Problem IV. Let $P \in \mathbb{R}$ be positive, and $\bar{\psi} \in \bar{H}_{0} \supset H^{1 / 2}(-1,1) / \mathscr{C}$. Find ${ }_{855} \bar{u}_{\mathrm{n}} \in H_{0}$ such that:

$$
\forall \hat{u} \in H_{0}, \quad \bar{a}\left(\hat{u}-\bar{u}_{\mathrm{n}}, \bar{u}_{\mathrm{n}}\right)+\phi(-\hat{u})-\phi\left(-\bar{u}_{\mathrm{n}}\right) \geq 0 .
$$

Setting $\tilde{t}_{\mathrm{n}}=\overline{\mathscr{L}}^{\prime}\left(\bar{u}_{\mathrm{n}}\right)$, the equivalence:

$$
-\bar{u}_{\mathrm{n}} \in \partial \phi^{*}\left[\tilde{t}_{\mathrm{n}}\right] \quad \Longleftrightarrow \quad \tilde{t}_{\mathrm{n}} \in \partial \phi\left[-\bar{u}_{\mathrm{n}}\right]
$$

shows the equivalence of problems IV' and IV. Thanks to propositions 19 and 21, the solutions $\bar{u}_{\mathrm{n}} \in H_{0}$ and $\tilde{t}_{\mathrm{n}} \in \bar{H}_{00}^{\prime}$ of problems IV and IV' provide the unique $\left(\bar{u}_{\mathrm{n}}, \tilde{t}_{\mathrm{n}}\right) \in H_{0} \times \bar{H}_{00}^{\prime}$ such that there exists $\tilde{F} \in \mathbb{R}$ such that:

$$
\begin{aligned}
& -\frac{1}{\pi} \tilde{t}_{\mathrm{n}} * \mathrm{pv} 1 / x+s \tilde{t}_{\mathrm{n}}=\bar{u}_{\mathrm{n}}^{\prime}, \\
& \bar{u}_{\mathrm{n}}-\frac{\int_{-1}^{1} t_{1} \bar{u}_{\mathrm{n}}}{\int_{0}^{1} t_{1}} \chi_{] 0,1[}-\bar{\psi}-\underset{]-1,1[}{\operatorname{ess} \sup }\left(\bar{u}_{\mathrm{n}}-\frac{\int_{-1}^{1} t_{1} \bar{u}_{\mathrm{n}}}{\int_{0}^{1} t_{1}} \chi_{] 0,1[}-\bar{\psi}\right) \leq 0, \\
& \tilde{t}_{\mathrm{n}}+\tilde{F} t_{1}-P t_{0} \leq 0, \\
& {\left[\bar{u}_{\mathrm{n}}-\frac{\int_{-1}^{1} t_{1} \bar{u}_{\mathrm{n}}}{\int_{0}^{1} t_{1}} \chi_{] 0,1[}-\bar{\psi}-\underset{]-1,1[}{\operatorname{ess} \sup }\left(\bar{u}_{\mathrm{n}}-\frac{\int_{-1}^{1} t_{1} \bar{u}_{\mathrm{n}}}{\int_{0}^{1} t_{1}} \chi_{] 0,1[}-\bar{\psi}\right)\right] \times} \\
& \times\left[\tilde{t}_{\mathrm{n}}+\tilde{F} t_{1}-P t_{0}\right]=0,
\end{aligned}
$$

$\mathbf{8 6 0}$ in $]-1,1\left[\right.$. We now must get rid of the unwanted term containing $\chi_{0,1}[$. This is going to be achieved in the next proposition by replacing first $\bar{\psi}$ by $\bar{\psi}-C \chi_{] 0,1}$, and then, performing a fixed point strategy. 
Proposition 22. Let $P \in \mathbb{R}$ be positive and $\bar{\psi} \in \bar{H}_{0}=H^{1 / 2}(-1,1) / \mathbb{R}+\mathbb{R} \chi_{] 0,1}$. Then, there exist $\left(\bar{u}_{n}, \tilde{t}_{\mathrm{n}}\right) \in H_{0} \times \bar{H}_{00}^{\prime}$ and $\tilde{F} \in \mathbb{R}$ such that:

$$
\begin{aligned}
& -\frac{1}{\pi} \tilde{t}_{\mathrm{n}} * \operatorname{pv} 1 / x+s \tilde{t}_{\mathrm{n}}=\bar{u}_{\mathrm{n}}^{\prime}, \\
& \bar{u}_{\mathrm{n}}-\bar{\psi}-\operatorname{ess} \sup \left(\bar{u}_{\mathrm{n}}-\bar{\psi}\right) \leq 0, \\
& \tilde{t}_{\mathrm{n}}+\tilde{F} t_{1}-P t_{0} \leq 0, \\
& {\left[\bar{u}_{\mathrm{n}}-\bar{\psi}-\underset{]-1,1[}{\operatorname{ess} \sup }\left(\bar{u}_{\mathrm{n}}-\bar{\psi}\right)\right]\left[\tilde{t}_{\mathrm{n}}+\tilde{F} t_{1}-P t_{0}\right]=0,}
\end{aligned}
$$

865 in $]-1,1[$.

Proof. In all the proof, we denote problems $\operatorname{IV}^{\prime}(C)$ and $\operatorname{IV}(C)$, the problems $\operatorname{IV}^{\prime}$ and IV, in which the data $\bar{\psi} \in \bar{H}_{0}$ has been replaced by $\bar{\psi}-C \chi_{] 0,1[}$.

Step 1. Let $u \in H^{1 / 2} / \mathbb{R}$ and $\tilde{t}=\overline{\mathscr{L}}^{\prime}(u)$. We have the identity:

$$
\int_{-1}^{1} t_{1} u=-\frac{2}{\pi} \int_{-1}^{1} t_{1}(\tilde{t} * \log |\cdot|) .
$$

We shall establish the result only in the case where $\tilde{t}$ happens to be an 870 integrable function, the general case being easily deduced from this particular case by a density argument. Using proposition 19, we have:

$$
\int_{-1}^{1} t_{1} u=\bar{b}\left(\tilde{t}, t_{1}\right)=-\frac{1}{\pi} \int_{-1}^{1} t_{1}(\tilde{t} * \log |\cdot|)+\int_{-1}^{1} t_{1} \int_{-1}^{x} s \tilde{t}
$$

where the integral notation makes sense since $\tilde{t}$ has been supposed to be an integrable function. Since $\tilde{t} \in \bar{H}_{00}^{\prime}$ and $s$ is constant over $]-1,0[$ and $] 0,1[$, we have:

$$
\int_{-1}^{x} s \tilde{t}=s(x) \int_{-1}^{x} \tilde{t}
$$

so that:

$$
\int_{-1}^{1} t_{1} \int_{-1}^{x} s \tilde{t}=\int_{-1}^{1} s t_{1} \int_{-1}^{x} \tilde{t}=-\int_{-1}^{1} \tilde{t} \int_{-1}^{x} s t_{1}=-\frac{1}{\pi} \int_{-1}^{1} t_{1}(\tilde{t} * \log |\cdot|),
$$

where the last equality comes from $\bar{b}\left(t_{1}, \tilde{t}\right)=0$. Hence:

$$
\int_{-1}^{1} t_{1} u=-\frac{2}{\pi} \int_{-1}^{1} t_{1}(\tilde{t} * \log |\cdot|) .
$$

Step 2. Let $C_{1}, C_{2} \in \mathbb{R}$, arbitrary, and $u_{1}, u_{2} \in H^{1 / 2} / \mathbb{R}$ denote the solutions of problems $I V\left(C_{1}\right)$ and $I V\left(C_{2}\right)$, respectively. Then, the following estimate holds true:

$$
\frac{\left|\int_{-1}^{1} t_{1}\left(u_{2}-u_{1}\right)\right|}{\int_{0}^{1} t_{1}} \leq \sqrt{2 P\left(s_{-}-s_{+}\right)} \sqrt{\left|C_{2}-C_{1}\right|} .
$$


Set $\tilde{t}_{1}=\overline{\mathscr{L}}^{\prime}\left(u_{1}\right)$ and $\tilde{t}_{2}=\overline{\mathscr{L}}^{\prime}\left(u_{2}\right)$. Thanks to step 1 and the Cauchy-Schwarz inequality, we have:

$$
\begin{aligned}
& \left|\int_{-1}^{1} t_{1}\left(u_{2}-u_{1}\right)\right| \leq \\
& \quad \sqrt{-\frac{1}{\pi} \int_{-1}^{1}\left(\tilde{t}_{2}-\tilde{t}_{1}\right)\left(\left(\tilde{t}_{2}-\tilde{t}_{1}\right) * \log |\cdot|\right)} \sqrt{-\frac{1}{\pi} \int_{-1}^{1} t_{1}\left(t_{1} * \log |\cdot|\right)} .
\end{aligned}
$$

But:

$$
-\frac{1}{\pi} \int_{-1}^{1}\left(\tilde{t}_{2}-\tilde{t}_{1}\right)\left(\left(\tilde{t}_{2}-\tilde{t}_{1}\right) * \log |\cdot|\right)=\bar{b}\left(\tilde{t}_{2}-\tilde{t}_{1}, \tilde{t}_{2}-\tilde{t}_{1}\right)
$$

and:

$$
-\frac{1}{\pi} \int_{-1}^{1} t_{1}\left(t_{1} * \log |\cdot|\right)=-\int_{-1}^{1} t_{1} \int_{-1}^{x} s t_{1}=\frac{s_{-}-s_{+}}{2}\left(\int_{0}^{1} t_{1}\right)^{2},
$$

so that:

$$
\frac{\left|\int_{-1}^{1} t_{1}\left(u_{2}-u_{1}\right)\right|}{\int_{0}^{1} t_{1}} \leq \sqrt{2\left(s_{-}-s_{+}\right) \bar{b}\left(\tilde{t}_{2}-\tilde{t}_{1}, \tilde{t}_{2}-\tilde{t}_{1}\right)},
$$

and there remains only to estimate $\bar{b}\left(\tilde{t}_{2}-\tilde{t}_{1}, \tilde{t}_{2}-\tilde{t}_{1}\right)$. To obtain it, we start with:

$$
\begin{gathered}
{\left[u_{2}-\frac{\int_{-1}^{1} t_{1} u_{2}}{\int_{0}^{1} t_{1}} \chi_{] 0,1[}-\bar{\psi}+C_{2} \chi_{] 0,1[}-\underset{]-1,1[}{\operatorname{ess} \sup }\left(u_{2}-\frac{\int_{-1}^{1} t_{1} u_{2}}{\int_{0}^{1} t_{1}} \chi_{] 0,1[}-\bar{\psi}+C_{2} \chi_{] 0,1[}\right)\right.} \\
\left.-u_{1}+\frac{\int_{-1}^{1} t_{1} u_{1}}{\int_{0}^{1} t_{1}} \chi_{] 0,1[}+\bar{\psi}-C_{1} \chi_{] 0,1[}+\underset{]-1,1[}{\operatorname{ess} \sup }\left(u_{1}-\frac{\int_{-1}^{1} t_{1} u_{1}}{\int_{0}^{1} t_{1}} \chi_{] 0,1[}-\bar{\psi}+C_{1} \chi_{] 0,1[}\right)\right] \times \\
\times\left[\tilde{t}_{2}+\tilde{F}_{2} t_{1}-P t_{0}-\tilde{t}_{1}-\tilde{F}_{1} t_{1}+P t_{0}\right] \leq 0
\end{gathered}
$$

in $]-1,1[$. By integration:

$$
\int_{-1}^{1}\left[\left(u_{2}-u_{1}\right)-\frac{\int_{-1}^{1} t_{1}\left(u_{2}-u_{1}\right)}{\int_{0}^{1} t_{1}} \chi_{] 0,1[}+\left(C_{2}-C_{1}\right) \chi_{] 0,1[}\right]\left[\tilde{t}_{2}-\tilde{t}_{1}+\left(\tilde{F}_{2}-\tilde{F}_{1}\right) t_{1}\right] \leq 0
$$

which is nothing but:

$$
\bar{b}\left(\tilde{t}_{2}-\tilde{t}_{1}, \tilde{t}_{2}-\tilde{t}_{1}\right)+\left(C_{2}-C_{1}\right)\left(\tilde{F}_{2}-\tilde{F}_{1}\right) \int_{0}^{1} t_{1} \leq 0 .
$$

885 Recalling that, for $i=1,2$ :

$$
-P\left(\int_{-1}^{0} t_{0}\right) \leq \tilde{F}_{i}\left(\int_{0}^{1} t_{1}\right) \leq P\left(\int_{0}^{1} t_{0}\right)
$$


the expected conclusion follows.

Step 3. For arbitrary $C \in \mathbb{R}$, consider problem $I V(C)$, its unique solution $u_{C} \in \bar{H}_{00} \simeq H_{0}$. Then:

$$
\exists M \in \mathbb{R}, \quad \forall C \in \mathbb{R}, \quad \frac{\left|\int_{-1}^{1} t_{1} u_{C}\right|}{\int_{0}^{1} t_{1}} \leq M .
$$

Let $C \in \mathbb{R}$ and $u_{C} \in H^{1 / 2}(-1,1) / \mathbb{R}$ the solution of problem $\operatorname{IV}(C)$. Setting soo $\tilde{t}_{C}=\overline{\mathscr{L}}^{\prime}\left(u_{C}\right) \in \bar{H}_{00}^{\prime}$, there exist $\delta \in \mathbb{R}$ and $F_{C}$ in the interval:

$$
-P\left(\int_{-1}^{0} t_{0}\right) /\left(\int_{0}^{1} t_{1}\right) \leq F_{C} \leq P\left(\int_{0}^{1} t_{0}\right) /\left(\int_{0}^{1} t_{1}\right),
$$

satisfying:

$$
\begin{aligned}
& -\frac{1}{\pi} \tilde{t}_{C} * \mathrm{pv} 1 / x+s \tilde{t}_{C}=u_{C}^{\prime} \\
& u_{C}-\bar{\psi}-\delta \chi_{] 0,1[} \leq 0 \\
& \tilde{t}_{C}+F_{C} t_{1}-P t_{0} \leq 0 \\
& {\left[u_{C}-\bar{\psi}-\delta \chi_{] 0,1[}\right]\left[\tilde{t}_{C}-F_{C} t_{1}-P t_{0}\right]=0}
\end{aligned}
$$

in $]-1,1[$. Setting:

$$
\overline{\bar{K}}_{00}^{\prime}\left(F_{C}\right) \stackrel{\text { def }}{=}\left\{\hat{t} \in \bar{H}_{00}^{\prime} \mid \hat{t}+F_{C} t_{1}-P t_{0} \leq 0\right\},
$$

$\tilde{t}_{C} \in \overline{\bar{K}}_{00}^{\prime}\left(F_{C}\right)$ is the unique solution of the variational inequality:

$$
\forall \hat{t} \in \overline{\bar{K}}_{00}^{\prime}\left(F_{C}\right), \quad \bar{b}\left(\tilde{t}_{C}, \hat{t}-\tilde{t}_{C}\right) \geq\left\langle\bar{\psi}, \hat{t}-\tilde{t}_{C}\right\rangle_{\bar{H}_{00}, \bar{H}_{00}^{\prime}} .
$$

The test function:

$$
\hat{t}_{0} \stackrel{\text { def }}{=} \mid \begin{aligned}
& \left.P t_{0}+t_{1}\left(\int_{-1}^{0} t_{0}\right) /\left(\int_{0}^{1} t_{1}\right), \quad \text { in }\right]-1,0[, \\
& \left.P t_{0}-t_{1}\left(\int_{0}^{1} t_{0}\right) /\left(\int_{0}^{1} t_{1}\right), \quad \text { in }\right] 0,1[,
\end{aligned}
$$

is independant of $C$ and belongs to $\overline{\bar{K}}_{00}^{\prime}\left(F_{C}\right)$. Using it in the variational inequality gives:

$$
\bar{b}\left(\tilde{t}_{C}, \tilde{t}_{C}\right) \leq \bar{b}\left(\tilde{t}_{C}, \hat{t}_{0}\right)+\left\langle\bar{\psi}, \hat{t}_{0}-\tilde{t}_{C}\right\rangle_{\bar{H}_{00}, \bar{H}_{00}^{\prime}},
$$

895 which entails that $\left\|\tilde{t}_{C}\right\|_{\bar{H}_{00}^{\prime}}$ is bounded by a constant independant of $C$. The same is therefore true of $\left\|u_{C}\right\|_{\bar{H}_{00}}$. This entails the expected result.

Step 4. The mapping:

$$
C \mapsto \frac{\int_{-1}^{1} t_{1} u_{C}}{\int_{0}^{1} t_{1}},
$$


defined for arbitrary $C \in \mathbb{R}$, has at least one fixed point which entails the proposition.

This function maps continuously $[-M, M]$ into itself so the conclusion follows from the Brouwer ${ }^{1}$ fixed point theorem and proposition 21.

As a corollary of proposition 22, we get the following theorem.

Theorem 23. Let $P \in \mathbb{R}$ be positive, $s:]-1,1[\rightarrow \mathbb{R}$ the function defined by $s=s_{-} \chi_{]-1,0[}+s_{+} \chi_{] 0,1[}$ with $s_{-}>s_{+}$and $\bar{\psi} \in H^{1 / 2}(-1,1)+\mathbb{R} \chi_{] 0,1[}$. Then, there exist $\bar{u}_{\mathrm{n}} \in H^{1 / 2}(-1,1)$ and $t_{\mathrm{n}} \in \bar{H}^{\prime}$ such that:

$$
\begin{aligned}
& \left.-\frac{1}{\pi} t_{\mathrm{n}} * \mathrm{pv} 1 / x+s t_{\mathrm{n}}=\bar{u}_{\mathrm{n}}^{\prime}, \quad \text { in }\right]-1,1[, \\
& \bar{u}_{\mathrm{n}}-\bar{\psi} \leq 0, \quad t_{\mathrm{n}} \leq 0, \quad\left(\bar{u}_{\mathrm{n}}-\bar{\psi}\right) t_{\mathrm{n}}=0, \\
& \left\langle t_{\mathrm{n}}, \chi_{]-1,1[}\right\rangle_{H^{-1 / 2}, H^{1 / 2}}=-P .
\end{aligned}
$$

Finally, if $\bar{\psi}$ is replaced by $\bar{\psi}+C(C \in \mathbb{R})$, then the corresponding solution is $\left(\bar{u}_{\mathrm{n}}+C, t_{\mathrm{n}}\right)$.

Remark. The example of the rigid flat punch (that is, the case $\bar{\psi} \equiv 0$ ) studied in the beginning of this section made it plausible that some kind of uniqueness should be expected. In the case $s_{-}<s_{+}$, uniqueness was a straightforward consequence of the coercivity of $\bar{b}$. In the case $s_{-}>s_{+}$, the situation is more involved since coercivity does not hold. Let us give an example of nonuniqueness of $t_{\mathrm{n}}$. Set:

$$
\begin{aligned}
t_{\mathrm{n}}(x) & =-P \frac{\left|t_{1}(x)\right|}{\int_{-1}^{1}\left|t_{1}(u)\right| \mathrm{d} u}, \\
\bar{u}_{\mathrm{n}}^{\prime} & \left.=-\frac{1}{\pi} t_{\mathrm{n}} * \mathrm{pv} 1 / x+s t_{\mathrm{n}}, \quad \text { in }\right]-1,1[.
\end{aligned}
$$

Choose $\bar{\psi} \equiv \bar{u}_{\mathrm{n}}$, so that the above defined $\left(\bar{u}_{\mathrm{n}}, t_{\mathrm{n}}\right) \in W^{1, p} \times L^{p}$ for all $p$ such that:

$$
\frac{1}{p}>\alpha=\frac{1}{\pi} \underset{] 0, \pi[}{\arctan }\left(-1 / s_{-}\right)-\frac{1}{\pi} \underset{] 0, \pi[}{\arctan }\left(-1 / s_{+}\right),
$$

provides a solution of the corresponding problem. But, it can be readily checked that $\left(\bar{u}_{\mathrm{n}}, t_{\mathrm{n}}+\beta t_{1} / \int_{-1}^{1}\left|t_{1}\right|\right)$ is also a solution, for all $\beta \in[-P, P]$.

The above construction fails in the case $\bar{\psi}$ is Lipschitz-continuous. It seems plausible that $\bar{u}_{\mathrm{n}}$ is generally unique, and that the same is true of $t_{\mathrm{n}}$ in the case of a Lipschitz-continuous obstacle. But I have not been able to prove it so far.

\footnotetext{
${ }^{1}$ of course, it was not necessary to invoke Brouwer's theorem here, but the choice has been made to make no reference to the ordering on the real line, so that the proof can be readily extended to the situation of an arbitrary finite number of jumps of the function $s$.
} 
The aim of the following theorem is to show that the asymptotic behaviour of $t_{\mathrm{n}}$ at $x=0$ encountered in the example at the beginning of the section, is universal. It is remarkable that the surface traction goes to zero at the jump of the friction coefficient whatever the shape of the (smooth) indentor is.

Theorem 24. Assume that $\bar{\psi}^{\prime}$ is piecewise Lipschitz-continuous. Let $\left(\bar{u}_{n}, t_{\mathrm{n}}\right)$ be a solution of the problem considered in theorem 23, such that the point $x=0$ where is the jump in the friction coefficient, is an interior point of the contact set (the support of $t_{\mathrm{n}}$ ). Then, the measure $t_{\mathrm{n}}$ behaves in the neighbourhood of $x=0$ as:

$$
t_{\mathrm{n}}=\frac{\bar{\psi}^{\prime}(0+)-\bar{\psi}^{\prime}(0-)}{s_{+}-s_{-}}-\frac{\theta|x|^{\beta}}{\sqrt{1+s^{2}(x)}},
$$

where

$$
\beta \stackrel{\text { def }}{=} \frac{1}{\pi} \underset{] 0, \pi[}{\arctan }\left(-1 / s_{-}\right)-\frac{1}{\pi} \underset{] 0, \pi[}{\arctan }\left(-1 / s_{+}\right),
$$

925 is in $] 0,1\left[\right.$ because of the condition $s_{+}<s_{-}$, and $\theta$ is a measure whose restriction to a neighborhood of $x=0$ is a function which is Hölder-continuous of exponent $1-\beta$.

Proof. Set:

$$
\bar{\phi}(x)=\bar{\psi}^{\prime}(0-) x \chi_{]-1,0[}(x)+\bar{\psi}^{\prime}(0+) x \chi_{] 0,1[}(x),
$$

where $\chi_{S}$ is the indicator function of the set $S$. We have the decomposition:

$$
t_{\mathrm{n}}=\overline{\mathscr{L}}^{\prime}(\bar{\phi})+\overline{\mathscr{L}}^{\prime}(\bar{\psi}-\bar{\phi})+\overline{\mathscr{L}}^{\prime}\left(\bar{u}_{\mathrm{n}}-\bar{\psi}\right)-P t_{0}+C t_{1},
$$

for some $C \in \mathbb{R}$. By hypothesis, $[-\epsilon, \epsilon](0<\epsilon<1)$ is contained in the interior of the contact set, and $\bar{\psi}^{\prime}$ is Lipschitz-continuous in $]-\epsilon, 0[$ and in $] 0, \epsilon[$. The distribution $\bar{u}_{\mathrm{n}}-\bar{\psi}$ is therefore supported in $]-1,1[\backslash]-\epsilon, \epsilon[$. We first focus on the first term of expression (22) and set:

$$
\begin{aligned}
\tau(x) & \stackrel{\text { def }}{=} \overline{\mathscr{L}}^{\prime}(\bar{\phi})(x) \\
& =\frac{s(x) \bar{\phi}^{\prime}(x)}{1+s^{2}(x)}-\frac{e^{\tau(x)}}{(1-x) \sqrt{1+s^{2}(x)}} \frac{1}{\pi} \oint_{-1}^{1} \frac{\bar{\phi}^{\prime}\left(x^{\prime}\right)\left(1-x^{\prime}\right) e^{-\tau\left(x^{\prime}\right)}}{\sqrt{1+s^{2}\left(x^{\prime}\right)}} \cdot \frac{\mathrm{d} x^{\prime}}{x^{\prime}-x},
\end{aligned}
$$

930 (up to an additive term of the form $C t_{1}$ that we bring together with the last term of (22)), thanks to proposition 19. The left limit and the right limit at $x=0$ of $\tau(x)$ can be computed by use of proposition 8 in [5]. They are found to have the same value:

$$
\lim _{x \rightarrow 0} \tau(x)=\frac{\bar{\psi}^{\prime}(0+)-\bar{\psi}^{\prime}(0-)}{s_{+}-s_{-}} .
$$

Therefore, the function $\tau$ is continuous at 0 . It is readily checked to have also 935 finite limits at $x=-1$ and $x=1$. Since its restriction to ]-1, $0[$ and to $] 0,1[$ is of class $C^{\infty}$, the distributional derivative $\tau^{\prime}$ is therefore an integrable function. 
Thanks to theorem 36 in appendix $\mathrm{C}$, the function $\tau$ (extended by 0 outside ]-1,1[) satisfies:

$$
\left.-\frac{1}{\pi} \tau * \operatorname{pv} 1 / x+s \tau=\bar{\phi}^{\prime}, \quad \text { in }\right]-1,1[.
$$

Taking the derivative (in the sense of distributions), we obtain:

$$
\left.-\frac{1}{\pi} \tau^{\prime} * \operatorname{pv} 1 / x+s \tau^{\prime}+\tau(0)\left(s_{+}-s_{-}\right) \delta=\left(\bar{\psi}^{\prime}(0+)-\bar{\psi}^{\prime}(0-)\right) \delta, \quad \text { in }\right]-1,1[,
$$

that is:

$$
\left.-\frac{1}{\pi} \tau^{\prime} * \operatorname{pv} 1 / x+s \tau^{\prime}=0, \quad \text { in }\right]-1,1[.
$$

Making use ${ }^{2}$ of theorem 36 in appendix C, we have:

$$
\tau^{\prime}(x)=C_{0} t_{0}+C_{1} t_{1},
$$

for some constants $C_{0}, C_{1} \in \mathbb{R}$, and therefore:

$$
\tau(x)=\tau(0)+\int_{0}^{x}\left(C_{0} t_{0}\left(x^{\prime}\right)+C_{1} t_{1}\left(x^{\prime}\right)\right) \mathrm{d} x^{\prime} .
$$

Hence, the claim in proposition 24 holds true at least for the term $\tau=\overline{\mathscr{L}}^{\prime}(\bar{\phi})$ of expression (22). Since $\bar{\psi}^{\prime}-\bar{\phi}^{\prime}$ is Lipschitz-continuous, $\overline{\mathscr{L}}^{\prime}(\bar{\psi}-\bar{\phi})$ is the 945 product of $t_{0}$ and some Hölder-continuous function of exponent $1-\beta$, thanks to proposition 19 in this article and proposition 7 in [5]. Hence, the claim in proposition 24 holds true at least for the sum of the first two terms of expression (22).

In the case where $\bar{u}_{\mathrm{n}}-\bar{\psi} \in W^{1, \infty}(-1,1), \overline{\mathscr{L}}\left(\bar{u}_{\mathrm{n}}-\bar{\psi}\right)$ is given by:

$$
\begin{aligned}
& \overline{\mathscr{L}}\left(\bar{u}_{\mathrm{n}}-\bar{\psi}\right)(x)=\frac{s(x)\left(u^{\prime}(x)-\bar{\psi}(x)\right)}{1+s^{2}(x)} \\
& -\frac{e^{\tau(x)}}{(1-x) \sqrt{1+s^{2}(x)}} \frac{1}{\pi} \oint_{-1}^{1} \frac{\left(u^{\prime}\left(x^{\prime}\right)-\bar{\psi}\left(x^{\prime}\right)\right)\left(1-x^{\prime}\right) e^{-\tau\left(x^{\prime}\right)}}{\sqrt{1+s^{2}\left(x^{\prime}\right)}} \cdot \frac{\mathrm{d} x^{\prime}}{x^{\prime}-x}+C^{\prime} t_{1},
\end{aligned}
$$

thanks to theorem 36 . In that case:

$$
\begin{aligned}
& \forall x \in]-\varepsilon, \varepsilon\left[, \quad \overline{\mathscr{L}}\left(\bar{u}_{\mathrm{n}}-\bar{\psi}\right)(x)=\right. \\
& -\frac{e^{\tau(x)}}{(1-x) \sqrt{1+s^{2}(x)}} \frac{1}{\pi} \int_{-1}^{1} \frac{\left(u^{\prime}\left(x^{\prime}\right)-\bar{\psi}\left(x^{\prime}\right)\right)\left(1-x^{\prime}\right) e^{-\tau\left(x^{\prime}\right)}}{\sqrt{1+s^{2}\left(x^{\prime}\right)}} \cdot \frac{\mathrm{d} x^{\prime}}{x^{\prime}-x}+C^{\prime} t_{1} .
\end{aligned}
$$

\footnotetext{
${ }^{2}$ we use actually a slightly stronger result, which is that $C_{0} t_{0}+C_{1} t_{1}$ are not only all the solutions of the homogeneous equation in $\cup_{p>1} L^{p}(-1,1)$ but also in the larger space of all the $L^{1}$ functions whose convolution with $\mathrm{vp} 1 / x$ is in $L^{1}$ (Hardy space). The proof of this stronger result is straightforward from the proof of theorem 36 .
} 
The first term in the second member is the product of $t_{0}$ with some function whose restriction to $]-\epsilon, \epsilon\left[\right.$ is of class $C^{\infty}$. Therefore, $C^{\prime}=-C$ since $t_{\mathrm{n}}$ is a nonpositive measure. Hence, the restriction to $]-\epsilon, \epsilon[$ of the last three terms in the second member of (22) equals the product of $t_{0}$ with some $C^{\infty}$ function. If $\bar{u}_{\mathrm{n}}-\bar{\psi} \notin W^{1, \infty}(-1,1)$, a straightforward limiting procedure shows that the same conclusion holds true. This is enough to conclude the proof.

\section{Appendix A: a generalization of the Lions-Stampacchia theorem}

Let $H$ be a Hilbert space with scalar product $(\cdot, \cdot)$ and norm $\|\cdot\|$. We are given a bilinear form $a(\cdot, \cdot)$ on $H \times H$ which is assumed to be:

- coercive: $\exists \alpha>0, \quad \forall v \in H, \quad a(v, v) \geq \alpha\|v\|^{2}$,

- continuous: $\exists M>0, \quad \forall u, v \in H, \quad a(u, v) \leq M\|u\|\|v\|$.

960 We are also given a function $\phi: H \rightarrow \mathbb{R} \cup\{+\infty\}$ which is assumed to be:

- proper: $\exists u \in H, \quad \phi(u) \neq+\infty$,

- convex: $\forall \lambda \in[0,1], \quad \forall u, v \in H, \quad \phi(\lambda u+(1-\lambda) v) \leq \lambda \phi(u)+(1-\lambda) \phi(v)$.

- lower-semicontinuous: $\forall u \in H, \quad \phi(u) \leq \liminf _{v \rightarrow u} \phi(v)$.

The following theorem is in the line of [10], chapter 2, section 8.5 or in [11], chapter II, section 3. Since the precise statement that is needed in this paper is not exactly found in these references, a proof is provided here for easy reference, but it brings nothing newer than the above references.

Theorem 25. There exists a unique $u \in H$ such that:

$$
\forall v \in H, \quad a(u, v-u)+\phi(v)-\phi(u) \geq 0 .
$$

Furthermore, in the particular case where $a(\cdot, \cdot)$ is symmetric, $u$ is characterised 970 by:

$$
\frac{1}{2} a(u, u)+\phi(u)=\min _{v \in H}\left\{\frac{1}{2} a(v, v)+\phi(v)\right\} .
$$

Remark. Let $K$ be a nonempty closed convex subset of $H$. Its indicator function $I_{K}$ (that is, the function that takes identically the value 0 in $K$ and $+\infty$ outside $K)$ is proper, lower-semicontinuous and convex. Let also $l \in H^{\prime}$ be a continuous linear form on $H$. In the particular case where $\phi=I_{K}-l$,

975 theorem 25 is nothing but the well-known Lions-Stampacchia theorem [1], and in the case where $K=H$, one recovers the Lax-Milgram theorem.

Proof. Note that the uniqueness of $u$ is obvious due to the coercivity of $a$. Let us first examine the particular situation where $a$ is symmetric. The epigragh:

$$
\text { epi } \phi=\{(v, \lambda) \in H \times \mathbb{R} \mid \phi(v) \leq \lambda\},
$$


is a nonempty (since $\phi$ is proper), closed (since $\phi$ is lower semicontinuous), convex ( since $\phi$ is convex) subset of $H \times \mathbb{R}$. Pick $\lambda_{0}<\phi\left(v_{0}\right)<+\infty$. By Hahn-Banach theorem, there exists a closed hyperplane that separates strictly the singleton $\left\{\left(v_{0}, \lambda_{0}\right)\right\}$ and epi $\phi$. In particular, there exists $\gamma \in \mathbb{R}$ and a continuous linear form $l \in H^{\prime}$ such that:

$$
\forall v \in H, \quad \phi(v) \geq\langle l, v\rangle_{H^{\prime}, H} \geq \beta\|v\|+\gamma,
$$

where $\beta=-\|l\|_{H^{\prime}}$. Therefore:

$$
\forall v \in H, \quad \frac{1}{2} a(v, v)+\phi(v) \geq \alpha\|v\|^{2}+\beta\|v\|+\gamma .
$$

Since $\alpha>0$ :

$$
\lim _{\|v\| \rightarrow+\infty}\left\{\frac{1}{2} a(v, v)+\phi(v)\right\}=+\infty
$$

and that function, being convex and lower semicontinuous attains its minimum, that is, there exists $u \in H$ such that:

$$
\frac{1}{2} a(u, u)+\phi(u)=\min _{v \in H}\left\{\frac{1}{2} a(v, v)+\phi(v)\right\} .
$$

This classically entails that 0 is in the subdifferential of that function at $u$ :

$$
\exists g \in \partial \phi[u], \quad \forall v \in H, \quad a(u, v-u)+(g, v-u)=0,
$$

where $\partial \phi[u]$ is the subdifferential of $\phi$ at $u$ (we recall that the subdifferential of the sum of a convex function and an everywhere continuous convex function is the sum of the subdifferentials, [12], corollary 2.63). But, from the definition of the subdifferential:

$$
\forall v \in H, \quad \phi(v)-\phi(u) \geq(g, v-u)
$$

and therefore:

$$
\forall v \in H, \quad a(u, v-u)+\phi(v)-\phi(u) \geq 0 .
$$

Hence the theorem is proved in the particular case where $a(\cdot, \cdot)$ is symmetric.

Let us turn to the general case. There exists a continuous linear operator $A: H \mapsto H$ such that:

$$
\forall u, v \in H, \quad a(u, v)=(A u, v) .
$$

It satisfies $\|A u\| \leq M\|u\|$. Let $\rho>0$ be a real constant to be fixed in the sequel, and $l_{1}, l_{2} \in H$. Thanks to the first part of the proof, there exists uniquely $u_{1}, u_{2} \in H$ such that:

$$
\forall v \in H, \quad\left(u_{i}, v-u_{i}\right)+\left(l_{i}, v\right)+\rho \phi(v)-\left(l_{i}, u_{i}\right)-\rho \phi\left(u_{i}\right) \geq 0,
$$

where $i=1,2$. We easily obtain:

$$
\left\|u_{2}-u_{1}\right\|^{2} \leq\left(l_{1}-l_{2}, u_{2}-u_{1}\right),
$$


which entails:

$$
\left\|u_{2}-u_{1}\right\| \leq\left\|l_{2}-l_{1}\right\|
$$

by the Cauchy-Schwarz inequality. Now, given an arbitrary $w \in H$, we denote by $u=P w$ the unique solution of:

$$
\forall v \in H, \quad(u, v-u)+(\rho A w-w, v)+\rho \phi(v)-(\rho A w-w, u)-\rho \phi(u) \geq 0,
$$

The nonlinear mapping $P: H \mapsto H$ satisfies the estimate:

$$
\left\|P w_{2}-P w_{1}\right\| \leq\left\|w_{2}-w_{1}-\rho\left(A w_{2}-A w_{1}\right)\right\|,
$$

and therefore:

$$
\begin{aligned}
\left\|P w_{2}-P w_{1}\right\|^{2} & \leq\left\|w_{2}-w_{1}\right\|^{2}-2 \rho\left(w_{2}-w_{1}, A w_{2}-A w_{1}\right)+\rho^{2}\left\|A w_{2}-A w_{1}\right\|^{2}, \\
& \leq\left(1-2 \rho \alpha+\rho^{2} M^{2}\right)\left\|w_{2}-w_{1}\right\|^{2} .
\end{aligned}
$$

The nonlinear mapping $P: H \mapsto H$ is therefore Lipschitz-continuous with modulus:

$$
k=\sqrt{1-2 \rho \alpha+\rho^{2} M^{2}} .
$$

Choosing $\rho \in] 0,2 \alpha / M^{2}$ [, we have $k<1$ and $P$ therefore has a unique fixed point $u$, thanks to the Banach fixed point theorem. It satisfies:

$$
\forall v \in H, \quad \rho(A u, v)+\rho \phi(v)-\rho(A u, u)-\rho \phi(u) \geq 0,
$$

and is therefore the expected solution.

\section{Appendix B: the spaces $H^{1 / 2}$ and $H^{-1 / 2}$}

We recall here for convenience some basic definitions and facts about the spaces $H^{1 / 2}$ and $H^{-1 / 2}$. The proofs of all the results (except for the last two ones) that are stated here are to be found in [13], chapter 1. Incidentally, the subtleties related to the use of the space $H_{00}^{1 / 2}$ seems to have escaped the attention of the contact mechanics community with the exception of [14].

Definition 26. For $a \in\{-\infty\} \cup \mathbb{R}$ and $b \in \mathbb{R} \cup\{+\infty\}$, the space:

$$
H^{1 / 2}(a, b) \stackrel{\text { def }}{=}\left\{u \in L^{2}(a, b) \mid \int_{a}^{b} \int_{a}^{b} \frac{|u(x)-u(y)|^{2}}{|x-y|^{2}} \mathrm{~d} x \mathrm{~d} y<\infty\right\},
$$

endowed with the natural norm:

$$
\|u\|_{H^{1 / 2}(a, b)} \stackrel{\text { def }}{=}\left(\int_{a}^{b}|u(x)|^{2} \mathrm{~d} x+\int_{a}^{b} \int_{a}^{b} \frac{|u(x)-u(y)|^{2}}{|x-y|^{2}} \mathrm{~d} x \mathrm{~d} y\right)^{1 / 2}
$$

is a Hilbert space. 
The step function:

$$
H(x)=\mid \begin{array}{ll}
0 & \text { if } x \in[-1,0[ \\
1 & \text { if } x \in[0,1]
\end{array}
$$

1020 is not in $H^{1 / 2}(-1,1)$. Therefore, the extension by zero of some $u \in H^{1 / 2}(-1,1)$ will not be, in general, in $H^{1 / 2}(\mathbb{R})$. Besides, we have:

$$
\log \log |x / 2| \in H^{1 / 2}(-1,1),
$$

which shows that $H^{1 / 2}(-1,1)$ contains noncontinuous or even unbounded functions.

With $u \in L^{1} \cap L^{2}$, the following definition:

$$
\mathscr{F}[u](t) \stackrel{\text { def }}{=} \frac{1}{\sqrt{2 \pi}} \int_{-\infty}^{+\infty} u(x) e^{i x t} \mathrm{~d} x,
$$

1025 is adopted for the Fourier transform, which can be extended, as usual, to all the tempered distributions.

Proposition 27. For $u \in H^{1 / 2}(\mathbb{R})$, the expression:

$$
\|u\|_{H^{1 / 2}(\mathbb{R})} \stackrel{\text { def }}{=}\left(\int_{-\infty}^{+\infty}(1+|t|)|\mathscr{F}[u](t)|^{2} \mathrm{~d} t\right)^{1 / 2}
$$

defines a norm on the space $H^{1 / 2}(\mathbb{R})$ which is equivalent to that of $H^{1 / 2}(\mathbb{R})$.

Theorem 28. The space $H^{1 / 2}(a, b)$ is (algebraically) the space of the restrictions 1030 to $] a, b\left[\right.$ of the functions in $H^{1 / 2}(\mathbb{R})$ :

$$
H^{1 / 2}(a, b)=\left\{U_{\mid] a, b[} \mid U \in H^{1 / 2}(\mathbb{R})\right\} .
$$

For $u \in H^{1 / 2}(a, b)$, the expression:

$$
\|u\|_{H^{1 / 2}(a, b)} \stackrel{\text { def }}{=} \inf \left\{\|U\|_{H^{1 / 2}(\mathbb{R})} \mid U_{\mid] a, b[}=u\right\}
$$

defines a norm on the space $H^{1 / 2}(a, b)$ which is equivalent to that of $H^{1 / 2}(a, b)$.

Theorem 29. The set $C_{0}^{\infty}(] a, b[)$ of the functions with infinitely many continuous derivatives and compact support in $] a, b\left[\right.$ is dense in $H^{1 / 2}(a, b)$ :

$$
H_{0}^{1 / 2}(a, b)=H^{1 / 2}(a, b) .
$$

1035 Definition 30. For $u \in H^{1 / 2}(a, b)$, we will denote by $\bar{u}$, the extension of $u$ by 0 outside $] a, b[$. We set:

$$
H_{00}^{1 / 2}(a, b) \stackrel{\text { def }}{=}\left\{u \in H^{1 / 2}(a, b) \mid \bar{u} \in H^{1 / 2}(\mathbb{R})\right\} .
$$


Theorem 31. The following identity holds true:

$$
H_{00}^{1 / 2}(a, b)=\left\{u \in H^{1 / 2}(a, b) \mid u(x) \frac{\sqrt{b-a}}{\sqrt{b-x} \sqrt{x-a}} \in L^{2}(a, b)\right\},
$$

and the space $H_{00}^{1 / 2}(a, b)$ endowed with the norm:

$$
\|u\|_{H_{00}^{1 / 2}(a, b)}=\left(\|u\|_{H^{1 / 2}(a, b)}^{2}+\left\|\frac{u \sqrt{b-a}}{\sqrt{b-x} \sqrt{x-a}}\right\|_{L^{2}(a, b)}^{2}\right)^{1 / 2} .
$$

is a Hilbert space.

1040 Definition 32. We denote by $H^{-1 / 2}(a, b)$ the dual space of $H^{1 / 2}(a, b)$.

Since the restriction to $] a, b\left[\right.$ of some function in $H^{1 / 2}(\mathbb{R})$ is in $H^{1 / 2}(a, b)$, then, on the dual side, the extension $\bar{u}$ of some $u \in H^{-1 / 2}(a, b)$ by 0 outside ]$a, b\left[\right.$ defines a distribution in $H^{-1 / 2}(\mathbb{R})$.

Proposition 33. For $u \in H^{-1 / 2}(a, b)$, the expression:

$$
\|u\|_{H^{-1 / 2}(a, b)} \stackrel{\text { def }}{=}\left(\int_{-\infty}^{+\infty} \frac{|\mathscr{F}[\bar{u}](t)|^{2}}{1+|t|} \mathrm{d} t\right)^{1 / 2}
$$

defines a norm on the space $H^{-1 / 2}(a, b)$ which is equivalent to that of $H^{-1 / 2}(a, b)$.

Based on theorem 31 , the restriction to $] a, b\left[\right.$ of some distribution in $H^{-1 / 2}(\mathbb{R})$ is not in $H^{-1 / 2}(a, b)$, in general, but in the larger space $H_{00}^{1 / 2^{\prime}}(a, b)$. Any $u \in H_{00}^{1 / 2^{\prime}}(a, b)$ can be written in the form:

$$
u=u_{0}+f_{1},
$$

with $u_{0} \in H^{-1 / 2}(a, b)$ and $f_{1}$ is a function such that $f_{1} \sqrt{b-x} \sqrt{x-a} / \sqrt{b-a} \in$ $1050 L^{2}(a, b)$.

Theorem 34 (Sobolev embeddings). Assume that $a$ and $b$ are finite. Then:

$$
\forall p \in\left[1, \infty\left[, \quad H^{1 / 2}(a, b) \subset L^{p}(a, b),\right.\right.
$$

with continuous embeddings. On the dual side:

$$
\forall p \in] 1, \infty], \quad L^{p}(a, b) \subset H^{-1 / 2}(a, b),
$$

with continuous embeddings.

Proposition 35. Assume that $a$ and $b$ are finite. Let $u \in \mathscr{M}([a, b])$ be a Radon 1055 measure (that is, an element of the dual space of $C^{0}([a, b])$ ). Suppose that in addition, $u \in H^{-1 / 2}(a, b)$ or $u \in H_{00}^{1 / 2^{\prime}}(a, b)$. Then the measure $u$ has no atom in $] a, b[$. 
Proof. Let $u \in \mathscr{M}([a, b]) \cap H^{-1 / 2}(a, b)$. The function $f$ defined by:

$$
f(x)=\mid \begin{array}{ll}
0, & \text { if } x<a, \\
\int_{[a, x]} u-\frac{x-a}{b-a} \int_{[a, b]} u, & \text { if } a<x<b, \\
0, & \text { if } x>b,
\end{array}
$$

is supported in $[a, b]$, has bounded variation and its distributional derivative 1060 equals the extension of $u$ by zero outside $[a, b]$. In addition, the function $f$ is in $H^{1 / 2}(a, b)$ (consider the Fourier transform of $f$ ). Having bounded variation, the function $f$ admits a left limit and a right limit for all $x_{0} \in[a, b]$. But, the integrability of the function:

$$
(x, y) \rightarrow\left(\frac{f(x)-f(y)}{x-y}\right)^{2},
$$

on a neighbourhood of $\left(x_{0}, x_{0}\right)$ requires that these left and right limits must be equal. The function $f$ is therefore a continuous function with bounded variation. Hence, its distributional derivative $u$ is a measure with no atom.

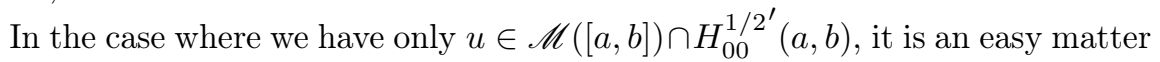
to build an extension of $u$ (still denoted by $u$ ) such that $u \in \mathscr{M}([a-1, b+1]) \cap$ $H^{-1 / 2}(a-1, b+1)$. Then, the expected conclusion follows from the first part of the proof.

\section{Appendix C: the Carleman singular integral equation with discontin- uous coefficient.}

Given $g \in L^{\infty}(-1,1)$ and $a \in W^{1, \infty}(-1,1)$, the Carleman singular integral equation is the problem of finding $f \in \cup_{p>1} L^{p}(-1,1)$ such that:

$$
\text { for a.a. } x \in]-1,1\left[, \quad a(x) f(x)-\frac{1}{\pi} \oint_{-1}^{1} \frac{f(t)}{t-x} \mathrm{~d} t=g(x),\right.
$$

1075 This linear equation was solved very elegantly by Carleman in 1922. The set of solutions is of dimension 1 , and the solution is unique when the total mass $\int_{-1}^{1} f$ has a prescribed value.

The extension of Carleman's analysis to the case where $a(x)$ is supposed only piecewise Lipschitz-continuous was performed in [5]. Surprisingly, the kernel (the vector space of solutions to the homogeneous equation), although still finitedimensional needs not to be of dimension 1 anymore. A precise proof of the following theorem is to be found in [5].

Theorem 36. Let $g \in L^{\infty}(-1,1 ; \mathbb{R})$ and $a:[-1,1] \rightarrow \mathbb{R}$ be some piecewise Lipschitz-continuous function. Take:

$$
\theta(x)=\mid \begin{array}{ll}
\underset{] 0, \pi[}{\arctan } \frac{-1}{a(x)}, & \text { if } x \in[-1,1], \\
0, & \text { if }|x|>1 .
\end{array}
$$


The function $\theta(x)$ is piecewise Lipschitz-continuous in $[-1,1]$ and we take:

$$
-1=\tilde{x}_{1}<\tilde{x}_{2}<\cdots<\tilde{x}_{m}=1
$$

to denote all its discontinuity points, and:

$$
-1<x_{1}<x_{2}<\cdots<x_{n}=1
$$

to denote all the discontinuity points $\tilde{x}_{i}$ which in addition satisfy the condition:

$$
\theta\left(\tilde{x}_{i}-0\right)>\theta\left(\tilde{x}_{i}+0\right) .
$$

Then, all the solutions $f \in \cup_{p>1} L^{p}(-1,1 ; \mathbb{R})$ of the singular integral equation:

$$
\text { for a.a. } x \in]-1,1\left[, \quad \frac{1}{\pi} \oint_{-1}^{1} \frac{f(t)}{t-x} \mathrm{~d} t+a(x) f(x)=g(x),\right.
$$

are given by:

$$
\begin{aligned}
f(x)=\frac{a(x) g(x)}{a^{2}(x)+1}-\frac{e^{\tau(x)}}{\sqrt{a^{2}(x)+1}} \frac{1}{\pi} \oint_{-1}^{1} \frac{g(t) e^{-\tau(t)}}{\sqrt{a^{2}(t)+1}} \cdot \frac{\mathrm{d} t}{t-x} & \\
& +\frac{P(x) e^{\tau(x)}}{\prod_{i=1}^{n}\left(x_{i}-x\right) \sqrt{a^{2}(x)+1}},
\end{aligned}
$$

where:

$$
\tau(x)=\frac{1}{\pi} \oint_{-1}^{1} \frac{\theta(t)}{t-x} \mathrm{~d} t,
$$

1090 and, $P \in \mathbb{R}_{n-1}[X]$ is some arbitrary real polynomial the degree of which is at most $n-1$.

Careful examination of the proof of theorem 36 in [5] shows that we also have:

$$
\forall k \in\{1,2, \ldots, n-1\}, \quad \int_{-1}^{1} \frac{e^{\tau(x)}}{\prod_{i=k}^{n}\left(x_{i}-x\right) \sqrt{a^{2}(x)+1}}=0 .
$$

\section{References}

1095 [1] J. L. Lions, G. Stampacchia, Variational inequalities, Communications on Pure and Applied Mathematics 20 (1967) 493-519.

[2] G. Duvaut, J. L. Lions, Les Inéquations en Mécanique et en Physique, Dunod, Paris, 1972.

[3] C. Eck, J. Jarušek, M. Krbec, Unilateral Contact Problems in Mechanics. Variational Methods and Existence Theorems., Monographs \& Textbooks in Pure \& Appl. Math. No. 270 (ISBN 1-57444-629-0). Chapman \& Hall/CRC, Boca Raton, 2005. 
[4] P. Ballard, Steady sliding frictional contact problems in linear elasticity, Journal of Elasticity 110 (1) (2013) 33-61.

1105

1110

[5] P. Ballard, J. Jarušek, Indentation of an elastic half-space by a rigid flat punch as a model problem for analyzing contact problems with coulomb friction, Journal of Elasticity 103 (2011) 15-52.

[6] L. A. Galin, Contact problems in the theory of elasticity, Moscow, 1953. English Translation by H. Moss, North Carolina State College, Department of Mathematics, 1961.

[7] M. Comninou, Stress singularity at a sharp edge in contact problems with friction, Zeitschrift für Angewandte Mathematik und Physik 27 (1976) 493-499.

[8] J. Dundurs, M. Comninou, Some consequences of the inequality conditions in contact and crack problems, Journal of Elasticity 9 (1) (1979) 71-82.

[9] P. Grisvard, Singularités en élasticité, Archive for Rational Mechanics and Analysis 107 (2) (1989) 157-180.

[10] J. L. Lions, Quelques Méthodes de Résolution des Problèmes aux Limites non Linéaires, Dunod, Paris, 1969.

[11] I. Ekeland, R. Temam, Convex Analysis and Variational Problems, SIAM, Philadelphia, 1999.

[12] V. Barbu, T. Precupanu, Convexity and Optimisation in Banach Spaces, Editura Academiei, Bucureşti and D. Reidel Publishing Company, Dordrecht/Boston/Lancaster (Third Edition, 1986), 1975.

[13] J. L. Lions, E. Magenes, Problèmes aux Limites non Homogènes et Applications, Volume 1, Dunod, Paris, 1968.

[14] C. Naéjus, A. Cimetière, A. Léger, A functional framework for the Signorini problem with Coulomb friction, in: M. Raous, M. Jean, J. J. Moreau (Eds.), Contact Mechanics, Plenum Press, New York and London, 1995, pp. 87-94. 